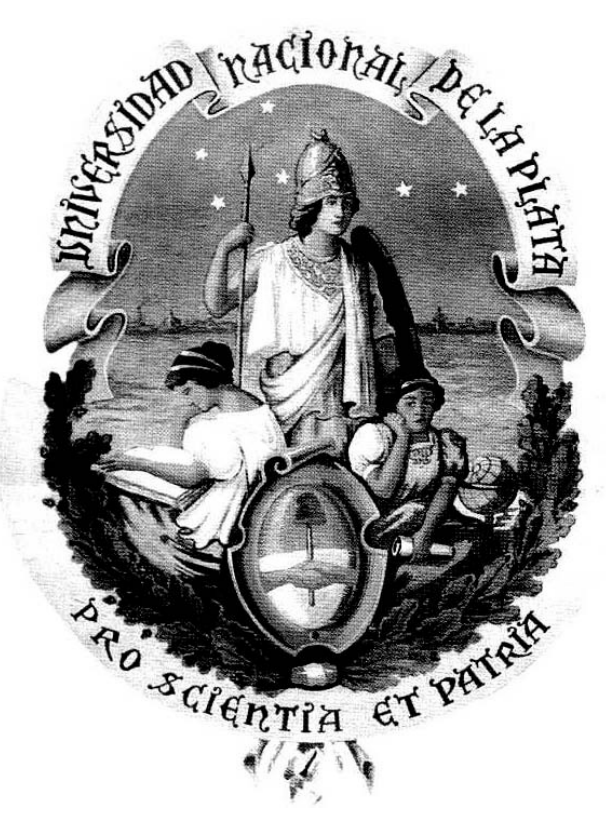

Universidad Nacional de La Plata

Facultad de Ciencias Astronómicas y Geofísicas

Tesis para obtener el grado académico de Doctor en Astronomía

\title{
SOBRE LA INTERACCIÓN PLANETA-DISCO EN DISCOS DE PLANETESIMALES
}

Lic. Pablo J. Santamaría

Director: Dr. Adrián Brunini 
Sobre la interacción planeta-disco en discos de planetesimales.

Pablo J. Santamaría.

Septiembre 2015.

Facultad de Ciencias Astronómicas y Geofísicas.

Universidad Nacional de La Plata. 


\section{Prefacio}

Esta tesis es presentada como parte de los requisitos para obtener el grado académico de Doctor en Astronomía de la Universidad Nacional de La Plata. La misma contiene los resultados de los estudios desarrollados bajo la dirección del Dr. Adrián Brunini en el seno del Grupo de Ciencias Planetarias ${ }^{1}$ de la Facultad de Ciencias Astronómicas y Geofísicas de la Universidad Nacional de La Plata.

Pablo Javier Santamaría.

e-mail: pablo@fcaglp.unlp.edu.ar

Sitio Web: http://gcp.fcaglp.unlp.edu.ar/integrantes:psantamaria:start La Plata, septiembre de 2015.

\footnotetext{
${ }^{1}$ Sitio Web: http://http://gcp.fcaglp.unlp.edu.ar/
} 



\section{Resumen}

En la actualidad, existe suficiente evidencia observacional proveniente de muchos de los sistemas planetarios extrasolares descubiertos en los últimos años, así como también resultados teóricos e indicios en nuestro propio sistema solar, para considerar que, en general, los planetas pueden no haberse formado en las órbitas actuales, sino que, de hecho, han migrado grandes distancias desde sus órbitas iniciales.

Esta tesis aborda algunos aspectos involucrados en uno de los posibles mecanismos dinámicos que puede originar una migración planetaria, a saber, la interacción gravitatoria de los planetas con un disco de planetesimales residuales del proceso de formación. Puesto que la magnitud y dirección de tal migración es determinada por una compleja interacción entre las diversas fuentes y sumideros dinámicos que aportan o restan partículas del disco a la región de interacción con el planeta, el proceso de migración resulta mejor comprendido a través de simulaciones numéricas. Ahora bien, para seguir la evolución del sistema sobre escalas de tiempos dinámicamente relevantes en tiempos de computo razonables, tales simulaciones consideran, usualmente, que el disco es modelado por un determinado número de partículas de masas iguales las cuales interaccionan gravitatoriamente con los planetas pero no interactúan entre ellas. Esta manera de simular el disco es objeto de estudio en esta tesis.

En primer lugar, los experimentos numéricos muestran la importancia de utilizar un número suficientemente grande de partículas para la modelización del disco de planetesimales si se quiere obtener una migración planetaria suave y no enteramente estocástica. En esta tesis abordamos, pues, un estudio sistemático del número de partículas adecuado para obtener resultados confiables en las simulaciones de la interacción planeta-disco, el cual mostró que cuando la "granularidad" del modelo (definida por la razón $M / m$ de la masa $M$ del planeta a la masa individual $m$ de cada planetesimal) es menor que cierto valor crítico, el efecto de la fricción dinámica entre las partículas del disco y el planeta es subestimado, lo cual conduce a un modelo incorrecto del problema. Cuando el número de partículas se incrementa, la granularidad aumenta y la acción de la fricción dinámica resulta relevante en la simulación.

Por otra parte, con la presencia de un planeta embebido en el disco con masa varios órdenes de magnitud mayor que la de los planetesimales, la interacción planetesimalplanetesimal, esto es, la autointeracción del disco, es típicamente despreciada en las simulaciones numéricas respecto a la interacción planeta-planetesimal. Sin embargo, la pérdida de excitación del disco por interacciones gravitaciones entre los planetesimales puede traer consecuencias en fenómenos como la captura en resonancias de movimientos medios. Así, en esta tesis, analizamos mediante un modelo analítico simplificado el efecto de la autointeracción del disco para determinar cual debería ser el número de partículas a utilizar en el modelo del disco para reproducir adecuadamente dicho fenómeno en una simulación que incluya la autointeracción. El resultado es que el número necesario genera un tiempo de cómputo prohibitivamente grande, pero permite expli- 
car por qué aun una simulación con granularidad correcta para modelar la migración planetaria no necesariamente es suficiente para describir las poblaciones resonantes si la autointeracción es considerada.

Claramente el estudio de los dos puntos anteriores requieren de un código numérico de $N$-cuerpos adecuado al problema de la interacción planeta-disco. En esta tesis presentamos la adaptación, a tal escenario, de un código originalmente desarrollado en la tesis de licenciatura del autor. Este código es un integrador simpléctico híbrido, capaz de resolver encuentros y colisiones entre cuerpos, donde el orden cero del problema se corresponde a un movimiento puramente kepleriano en torno a la estrella central, en tanto las interacciones gravitatorias entre cuerpos es computada a través de la estrategia del denominado código de árbol octal. Este último punto es el que permite la inclusión de un gran número de planetesimales para modelar el disco. Con el fin de testear la capacidad del código ampliado consideramos tres escenarios de migración planetaria presentes en la literatura. El primer escenario consistió en el estudio de la migración planetaria de un único cuerpo planetario sumergido en un disco de planetesimales de masa nebular mínima y densidad superficial constante prestando especial atención a su efecto sobre la estructura global del disco, según el trabajo de Cionco y Brunini (2002). Nuestro código reprodujo satisfactoriamente los resultados previamente obtenidos por dichos investigadores para un cuerpo de la masa de Júpiter, donde la migración procede hacia adentro con un tiempo de deriva característico del orden de $10^{6}$ años. Aumentando el grado de complejidad, un segundo escenario abordado fue el de los cuatro planetas gigantes exteriores en un disco de planetesimales con un perfil de densidad típico de los discos protoplanetarios. Las simulaciones numéricas (Fernández e Ip, 1984) muestran que Saturno, Urano y Neptuno migran, sobre el promedio, hacia afuera, mientras que Júpiter migra levemente hacia adentro. Ahora bien, dependiendo de la masa del disco, las simulaciones muestran que Neptuno puede tener una migración limitada a unas pocas UA, o bien sufrir una migración que lo lleva hasta el borde del disco e incluso, si el disco es lo suficiemente masivo, puede experimentar una migración en fuga con un semieje orbital que no varía en forma monótona (Gomes y otros, 2004). Con el fin de estudiar tal escenario consideramos un disco masivo extendiéndose hasta unas 50 UA para una configuración inicial de los planetas más compacta que la actual. Nuestro código nuevamente resultó exitoso, reproduciendo los resultados de Gomes y otros. Finalmente, el tercer escenario abordado es el denominado modelo de Niza. Este modelo considera una configuración compacta de los cuatro planetas, con un disco comenzando después del último planeta y extendiéndose hasta unas 30 UA. La compacticidad del sistema permite el cruce de la resonancia de movimientos medios 1:2 mutua de Júpiter y Saturno durante el proceso de migración lo que origina una desestabilización de todo el sistema conduciendo a Neptuno al interior del disco con la consecuente dispersión de los planetesimales del mismo, de manera tal que el modelo puede $(i)$ reproducir los elementos orbitales actuales de los planetas gigantes (Tsiganis y otros, 2005), (ii) mostrar que es posible la captura de las poblaciones de troyanos de Júpiter y Neptuno (Morbidelli y otros, 2005; Tsiganis y otros, 2005), (iii) dar una explicación al denominado bombardeo intenso tardío sobre los planetas interiores (Gomes y otros, 2005) y (iv) reproducir las principales características de la región transneptuniana (Levison y otros, 2008). Nuestro código nuevamente pudo reproducir el escenario propuesto para el bombardeo intenso tardío de Gomes y otros (2005). 


\section{Originalidad}

Las ideas, desarrollos, código computacional y resultados obtenidos son originales del autor y del director de la Tesis, salvo explícita mención. Los resultados del capítulo 4 fueron presentados en la Reunión 49 (2006) de la Asociación Argentina de Astronomía y publicados en un artículo pertinente (Planetary and Space Science, 55 (2007), pp. 21212127), en coautoría con A. Brunini, C. Cionco y H. Viturro. Algunos resultados del capítulo 5 se presentaron en la Reunión 49 ya mencionada y permanecen sin publicar, a la fecha, en revistas internacionales. 



\section{Índice general}

$\begin{array}{lll}\text { Prefacio } & \text { III }\end{array}$

Resumen $\quad$ V

$\begin{array}{lll}\text { Originalidad } & \text { VII }\end{array}$

1. Introducción: Relevancia de la interacción planeta-disco 1

2. Integrador numérico $\quad 7$

2.1. Introducción. . . . . . . . . . . . . . . . 7

2.2. Integradores simplécticos. . . . . . . . . . . . . . . . . 8

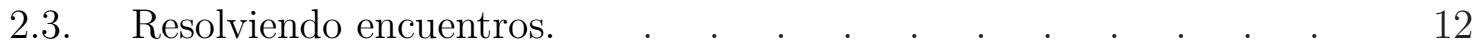

2.4. El algoritmo de árbol jerárquico octal. . . . . . . . . . 14

2.5. Detectando encuentros y colisiones. . . . . . . . . . 15

2.6. Desempeño del código. . . . . . . . . . . . . . . . . . 16

2.7. Modificaciones al código para problemas de interacción planeta-disco. 19

3. Escenarios de migración planetaria por interacción planeta-disco $\quad 21$

3.1. Introducción. . . . . . . . . . . . . . . . 21

3.2. Migración de un único cuerpo planetario. . . . . . . . . . 21

3.3. Migración planetaria en un disco masivo. . . . . . . . . . 29

3.4. $\quad$ El modelo de Niza. . . . . . . . . . . . . . . . . . . . 36

4. La importancia del número de partículas 41

4.1. Introducción. . . . . . . . . . . . . . . . . . 41

4.2. Planteo del problema. . . . . . . . . . . . . . . 41

4.3. Sensibilidad al número de partículas. . . . . . . . . . . 42

4.4. El término de fricción dinámica y su correcto modelado. . . 43

4.5. Simulaciones confiables y el número de partículas. . . . . . 45

4.6. Relevancia de los resultados. . . . . . . . . . . . 46

5. El efecto de la autointeracción del disco de planetesimales $\quad 49$

5.1. Introducción. . . . . . . . . . . . . . . . . . 49

5.2. Resonancias de movimiento medio y captura en resonancia. . . 51

5.3. Autoexcitación del disco de planetesimales. . . . . . . . 57

5.4. Disco de partículas de masas iguales. . . . . . . . . . 62

5.5. Disco de partículas de masas iguales y densidad superficial constante. 65 
6. Conclusiones y perspectivas $\quad 69$

A. Elementos de la formulación hamiltoniana de la mecánica clásica $\quad 71$

B. Variables democráticas heliocéntricas en el problema de $N$-cuerpos 81

C. La nebulosa solar de masa mínima $\quad 83$

D. Condición para las resonancias de Lindblad y de corrotación $\quad 85$

$\begin{array}{ll}\text { E. Zona de cruce planetaria } & 91\end{array}$

F. Evolución de la velocidad en el modelo "partícula en una caja" $\quad 93$

$\begin{array}{ll}\text { Bibliografía } & 95\end{array}$ 


\section{Capítulo 1}

\section{Introducción: Relevancia de la interacción planeta-disco}

En la actualidad existe suficiente evidencia para afirmar que los planetas se forman en discos de acreción protoplanetarios constituidos de gas y polvo orbitando en torno a una protoestrella, siendo tales discos un subproducto posible de la formación de la estrella central surgida del colapso gravitacional de densas nubes moleculares presentes en nuestra galaxia (Kortenkamp y otros, 2000; Cole y Woolfson, 2002; Armitage, 2010). Confirmación de tal estado inicial surge de la observación de discos alrededor de estrellas jóvenes ya sea en forma directa, a través de imágenes obtenidas por el telescopio espacial Hubble $u$ otros sistemas ópticos en tierra, o inferidos en forma indirecta, a partir de los excesos infrarrojos de sus espectros estelares (ver referencias anteriores). Además, la observación de estrellas jóvenes permite inferir que el tiempo de vida de estos discos es menor a $10^{7}$ años (Cole y Woolfson, 2002).

Un disco protoplanetario está compuesto principalmente de gas, pero también contiene partículas de polvo y hielo. Ahora bien, varios órdenes de magnitud en masa separan a las partículas de polvo de un cuerpo planetario. La evolución de una en otra supone un proceso de diversas etapas o regímenes de crecimiento, las cuales presentamos muy sucintamente a continuación (ver, por ejemplo, Armitage (2010), para una discusión más completa):

I. Coagulación colisional de granos de polvo microscópicos para formar agregados macroscópicos del tamaño de centímetros a metros y, finalmente, objetos con tamaños del orden de los kilómetros con masas del orden de $10^{12}-10^{18} \mathrm{~g}$ denominados planetesimales.

œ La dinámica de los granos de polvo inmersos en el disco está fuertemente influenciada por su interacción con el gas a través de la fuerza de fricción aerodinámica que experimentan, la cual conduce, por un lado, a un decaimiento diferenciado de las partículas al plano medio del disco (donde los granos más pequeños tardan más en depositarse que los grandes) y, por otro, a un decaimiento orbital diferenciado hacia la estrella (donde los granos más pequeños se dirigen hacia la estrella más rápidamente que los grandes). Ambas situaciones favorecen las colisiones entre los granos y, así, la posibilidad de aglomeramiento en objetos de tamaños cada vez mayores. Una vez que, eventualmente, los objetos alcanzan el tamaño de kilómetros, esto es, resultan en planetesimales, las fuerzas de fricción del gas son de menor relevancia sobre su 
dinámica y régimen de crecimiento que la mutua interacción gravitatoria entre planetesimales, que es, entonces, la que domina el proceso dando lugar a la segunda etapa de crecimiento. Un punto crucial es, dado que objetos creciendo del tamaño de centímetros a metros tienen el menor tiempo de decaimiento orbital y éste es de unos cientos de años a $1 \mathrm{UA}$, el proceso de formación de planetesimales tiene que ser más rápido, de lo contrario la mayoría del material sólido del disco decaería hacia la estrella central produciéndose una catastrófica pérdida de masa al evaporarse en las más calientes regiones internas del disco protoplanetario.

II. Acumulación gravitacional de planetesimales por colisiones directas en cuerpos con masas entre $10^{26}-10^{27} \mathrm{~g}$, denominados embriones planetarios.

$↔$ Debido a la atracción gravitatoria mutua, los cuerpos de mayor masa disminuyen su velocidad relativa respecto a los de menor masa y este proceso de fricción dinámica favorece el aumento gravitatorio de la sección eficaz de colisión, fenómeno denominado enfocamiento gravitacional. Así, los más grandes planetesimales presentes en cierta región crecen a expensas de sus vecinos. Simulaciones estadísticas y de $N$-cuerpos muestran que una población de planetesimales a 1 UA del cuerpo central y sobre una estrecha franja en semieje mayor, interactúan de manera tal que un cuerpo crece mucho más rápidamente separándose del resto de la población y convirtiéndose en un embrión planetario con un rango de masa entre Mercurio y Marte en una escala de tiempo de unos $10^{5}$ años. Típicamente tal embrión es unas $10^{2}-10^{3}$ veces más masivo que el segundo cuerpo mas grande de la región. Este régimen de crecimiento es conocido como etapa de crecimiento en fuga (runaway grow) ${ }^{1}$. Cuando en la vecindad del embrión los planetesimales comienzan a excitarse lo suficiente, esta etapa deja de ser eficiente y las simulaciones numéricas muestran una transición a un nuevo régimen de crecimiento autolimitado, llamado crecimiento oligárquico. En este régimen los embriones más grandes crecen más rápidamente que los más pequeños (aunque a tasas más lentas que en la etapa de crecimiento en fuga), conduciendo a una distribución de masa con embriones de masas típicamente entre la lunar y la de Marte embebidos en un enjambre de planetesimales.

III. Crecimiento caótico de los embriones planetarios por grandes impactos conduciendo a objetos de masas entre $10^{27}-10^{28} \mathrm{~g}$, que son los planetas terrestres o progenitores de los núcleos de planetas gigantes.

$↔$ Al final de la etapa de crecimiento oligárquico, los embriones han vaciado lo suficiente sus vecindades como para que la fricción dinámica no pueda mantener sus excentricidades e inclinaciones bajas: en consecuencia, la interacción gravitacional entre los mismos puede conducir a órbitas que se cruzan y así, ocasionalmente, al impacto entre dos embriones planetarios para formar un cuerpo mayor. Tales impactos continúan hasta que unos pocos planetas terrestres resultan aislados unos de otros en órbitas estables. La escala de tiempo de esta etapa es del orden de $10^{8}$ años.

IV. Los núcleos más masivos adquieren envolturas gaseosas del gas presente en el disco para formar los planetas gigantes gaseosos (como lo son Júpiter y Saturno, en nuestro sistema solar).

$\circlearrowleft$ Una vez que los planetas adquieren una masa del orden de una masa terrestre la interacción con el gas presente en el disco vuelve a jugar un papel relevante, pero no ya vía fricción, sino gravitacionalmente. Para un núcleo con masa del orden de diez masas terrestres,

\footnotetext{
${ }^{1}$ Una simulación de este régimen es detallada en 2.6.
} 
la interacción con el gas resulta tan fuerte que éste acreta gas del disco y un planeta gigante gaseoso es formado en una escala de tiempo de $10^{5}$ años.

Si bien el modelo descrito puede dar cuenta de la formación de los planetas gigantes gaseosos como Júpiter y Saturno en las posiciones que ocupan en nuestro sistema solar, no puede, sin embargo, formar a los gigantes helados, Urano y Neptuno, in situ, puesto que las escalas de tiempo resultan mucho mayores a la edad del sistema solar (Armitage, 2010). Asimismo, el modelo no puede explicar las inesperadas características de muchos de los exoplanetas descubiertos ya sea en órbitas muy próximas a la estrella central o con grandes excentricidades. Una manera de solventar tales escollos y reconciliar la teoría con las observaciones es suponer la posibilidad de que los planetas se pueden haber formado en regiones del disco que no son las que ocupan actualmente para luego, mediante ciertos mecanismos dinámicos, modificar sustancialmente sus órbitas hasta alcanzar las actuales, proceso denominado migración planetaria. Tres son los principales mecanismos dinámicos que pueden originar tal migración orbital.

I. Interacción entre el planeta y la componente gaseosa del disco protoplanetario.

Embebido en el disco, el potencial gravitatorio de un cuerpo suficientemente masivo, como lo es un planeta terrestre $y$, sobre todo, un gigante gaseoso, origina perturbaciones relevantes en la distribución de densidad del gas en ciertas localizaciones dadas por las resonancias de Lindblad, excitando allí ondas de densidad que transfieren momento angular del planeta al disco hacia zonas cada vez más alejadas. Esta interacción gravitacional con el disco tiende a repeler el gas localizado en la vecindad de la órbita del planeta y, si el planeta es lo suficientemente masivo, se forma una gran brecha anular en el gas. Una asimetría intrínseca entre la intensidad de los torques de las resonancias externas e internas al planeta originan una pérdida neta de momento angular del planeta lo que conduce a una disminución del semieje orbital, esto es, a una migración hacia el interior del disco (Goldreich y Tremaine, 1980) ${ }^{2}$. Esta migración de un planeta por la interacción con el gas presente en el disco protoplanetario constituye una explicación para la existencia de los exoplanetas denominados "Júpiter calientes" (hot Jupiters) (Lin, Bodenheimer y Richardson, 1996), los cuales son cuerpos de masa mínima ${ }^{3}$ mayor que la décima parte de la masa de Júpiter y localizados a menos de 0.1 UA de la estrella central.

II. Interacción entre el planeta y un disco de planetesimales residuales.

Un planeta puede también intercambiar momento angular interactuando gravitacionalmente con los planetesimales remanentes del proceso de formación planetaria. Un cuerpo planetario embebido en un disco de planetesimales ejerce al moverse fuerzas sobre el disco y la reacción a tales fuerzas influyen en su movimiento, de este modo, el planeta puede considerarse que se mueve como si estuviera en presencia de un medio resistivo producto de las interacciones gravitacionales con el mismo, y de aquí el nombre de fricción dinámica del proceso. Aquellos planetesimales que, debido de la interacción gravitatoria con el planeta, sean dispersados hacia la región exterior de la

\footnotetext{
${ }^{2}$ Una más extensa discusión de la migración planetaria en discos gaseosos es considerada en 3.2.

${ }^{3}$ Dado que las técnicas observacionales, en general, no pueden determinar la inclinación $i$ de la órbita del planeta, sólo puede derivarse la denominada masa mínima $M$ sen $i$ en vez de su masa $M$. Suponiendo que la orientación de las órbitas tiene una distribución aleatoria uniforme sobre su rango, la masa mínima en promedio es $\langle 1 / \operatorname{sen} i\rangle=\pi / 2$ veces menor que la masa real.
} 
órbita planetaria, producirán un decaimiento en el semieje mayor del mismo, mientras que, si la dispersión es hacia el interior, se producirá un incremento del semieje mayor. Ahora bien para un cuerpo planetario sumergido en el disco de planetesimales, los mismos provienen de cualquier dirección y por lo tanto, es de esperarse sólo pequeños cambios en la evolución temporal del semieje mayor. Sin embargo, si los sumideros y/o fuentes de tales planetesimales, como la eyección, transferencia de un planeta a otro e incluso poblaciones en resonancias de movimientos medios, se encuentran a un lado $\mathrm{u}$ otro de la órbita del planeta en cuestión, puede haber un flujo neto de material en una u otra dirección y el planeta se moverá en la dirección opuesta para conservar el momento angular del sistema, pudiéndose producir, así, una migración orbital sustancial. De hecho, el trabajo pionero de Fernández e Ip (1984) muestra que el sistema solar exterior favorece una importante migración hacia afuera de Urano y Neptuno, en tanto que Saturno también se dirige hacia el exterior pero en menor medida, mientras que Júpiter migra levemente hacia adentro. La clave de este proceso se debe a que Urano y Neptuno no eyectan eficientemente planetesimales del sistema, sino que la gran mayoría son dispersados hacia la región interior donde se encuentran Saturno y Júpiter. Así, Neptuno y Urano, por reacción, se mueven hacia afuera. Por otro lado, Júpiter eyecta fuera del sistema la gran mayoría de los planetesimales que éste encuentra y, por tanto, se mueve hacia al interior. Nótese que este escenario de migración de los planetas gigantes permiten que ellos puedan ser formados en una configuración más compacta y próxima al Sol, aminorando sustancialmente el problema de la formación de Urano y Neptuno in situ. Por otra parte, una migración hacia afuera de Neptuno juega un papel relevante para explicar la dinámica orbital de Plutón y la de objetos similares del cinturón de Kuiper (Malhotra, 1993, 1995) y muchas otras características de la arquitectura del sistema solar exterior según el denominado modelo de Niza (Tsiganis y otros, 2005; Morbidelli y otros, 2005; Levison y otros, 2008) ${ }^{4}$.

III. Interacción entre dos o más planetas en un sistema gravitatoriamente inestable.

La configuración de los planetas de un recién formado sistema planetario no necesariamente tiene que ser gravitacionalmente estable y la interacción gravitatoria entre los mismos puede conducir a órbitas que favorezcan los encuentros próximos, lo cual, por un lado, puede dar lugar a colisiones físicas, y, por otro, a la eyección de uno o más cuerpos, típicamente los de menor masa, dejando a los planetas sobrevivientes en órbitas excéntricas. Este último proceso de dispersión gravitacional planeta-planeta puede ser una explicación a las grandes excentricidades observadas en muchos de los planetas gigantes de los sistemas extrasolares descubiertos (Rasio y Ford, 1996; Weidenschilling y Marzari, 1996; Lin e Ida, 1997) y un posible origen para algunos de los llamados planetas interestelares (free-floating planets) observados en la nebulosa de Orión (Lucas y Roche, 2000) y en otras regiones.

En esta tesis nos interesa abordar los aspectos involucrados en las simulaciones del segundo escenario planteado, esto es, de la interacción planeta-disco de planetesimales una vez que la componente gaseosa del disco protoplanetario ya no está presente. En estas circunstancias, el escenario se corresponde a un problema $N$-cuerpos puramente gravitatorio, conformado por los planetas y un disco simulado típicamente con cierto

\footnotetext{
${ }^{4}$ Una discusión más extensa de estos modelos y su contexto observacional y teórico es dada en 3.3 y 3.4 .
} 
número de partículas de igual masa. Así las órbitas de tales cuerpos son calculadas integrando numéricamente sus ecuaciones de movimiento. Ahora bien, la dinámica de tales cuerpos está dominada por la fuerza del cuerpo central, con órbitas desviándose de un movimiento puramente kepleriano por fuerzas perturbativas debida a los otros cuerpos presentes, excepto cuando ocurra un encuentro, en cuyo caso la interacción entre los cuerpos involucrados puede dejar de ser un término perturbativo respecto a la fuerza central e incluso puede derivar en una colisión física. Por otra parte, existe una jerarquía de masas en el sistema: mientras que las partículas conforman el disco de planetesimales, los planetas constituyen objetos embebidos en el disco con masas de varios órdenes de magnitud mayores. En estas circunstancias la interacción entre planetesimales, la autointeracción del disco, puede ser despreciada, y las simulaciones proceden considerando a los planetesimales interactuando con los planetas pero no entre ellos. Finalmente debe tenerse presente que la mayor parte del tiempo de cómputo en las simulaciones de $N$-cuerpos reside en el cálculo de las interacciones gravitatorias mutuas, el cual, si se procede con una evaluación directa, crece con el cuadrado del número de cuerpos presentes y, por lo tanto, resulta de interés utilizar esquemas de evaluación menos costosos (a expensas de cierta pérdida de precisión). Debería, pues, resultar claro que la escritura de un código adecuado para la integración de las ecuaciones de movimiento teniendo en cuenta todas estas características no es trivial.

En esta tesis consideramos, entonces, en el capítulo 2, la adaptación al escenario de interacción planeta-disco de un integrador numérico, desarrollado originalmente en la tesis de licenciatura del autor, a partir de diversas ideas presentes en la literatura. A continuación, en el capítulo 3 consideramos diversos escenarios de migración de interés con el fin de poner a prueba la capacidad del código desarrollado. En el capítulo 4 realizamos un estudio sistemático sobre el número de partículas adecuado para describir el disco de planetesimales de manera que las simulaciones resulten confiables y en el capítulo 5 exploramos cuál debería ser el número de partículas a considerar en una simulación que reproduzca adecuadamente fenómenos como la captura en resonancias de movimientos medios si la autointeracción es incluida. Finalmente, el capítulo 6 está dedicado a las conclusiones y perspectivas de trabajo futuras. 



\section{Capítulo 2}

\section{Un integrador numérico para la simulación de la dinámica de discos de planetesimales}

\subsection{Introducción.}

Un integrador numérico del problema de $N$-cuerpos adecuado a la simulación de la dinámica de planetesimales en discos keplerianos debe, idealmente, satisfacer o aprovechar los siguientes puntos:

I. El integrador debe ser un integrador simpléctico para así preservar la evolución dinámica del sistema conservativo sobre largos tiempos.

II. Para un disco kepleriano existe un cuerpo central (el Sol, digamos) mucho más masivo que el resto de los cuerpos del sistema. En consecuencia, el integrador debería tomar en cuenta que los cuerpos, en ausencia de encuentros próximos, se mueven principalmente bajo un potencial central y la evaluación de tal interacción puede entonces efectuarse con la formulación analítica del problema de los dos cuerpos.

III. Encuentros próximos deben ser detectados e integrados con suficiente precisión. Esto es muy importante para la compresión de muchos procesos que afectan la estructura dinámica del sistema.

IV. El cálculo en forma directa de las interacciones gravitatorias mutuas debería ser evitado, puesto que el número de operaciones involucrado en este cálculo se incrementa con el cuadrado del número de cuerpos del sistema.

Nuestro propósito fue desarrollar un código que satisfaga tales requisitos utilizando, en conjunto, las soluciones propuestas en la literatura para cada uno de los puntos individuales. De esta manera, utilizamos un esquema de integración simpléctico descrito por Duncan y otros (1998), el cual satisface los dos primeros puntos. Para resolver el tercer punto, respecto a los encuentros, incorporamos el esquema descrito por Chambers (1999). Finalmente, para evitar el cómputo directo de las interacciones entre los cuerpos utilizamos un código de árbol, como es descrito por Brunini y Viturro (2003). El integrador así obtenido, bautizado como DAEDALUs, fue desarrollado inicialmente 
en el contexto de la tesis de licenciatura y una discusión exhaustiva del mismo puede verse en Santamaría (2004). En lo que sigue describimos brevemente los problemas involucrados y las soluciones propuestas, para luego discutir las modificaciones realizadas al código en el marco de esta tesis, esto es, estudiar problemas de interacción planeta-disco.

\subsection{Integradores simplécticos.}

El problema de $N$-cuerpos involucra un sistema hamiltoniano cuyas ecuaciones de movimiento constituyen un sistema de ecuaciones diferenciales de primer orden, el cual, sujeto a ciertas condiciones iniciales, define un problema de valor inicial para un sistema de ecuaciones diferenciales ${ }^{1}$. Es sabido que, en la práctica, la solución exacta de un problema de valor inicial solo puede ser determinada para un número más bien reducido de casos (y ésta no es, ciertamente, la situación del problema general de $N$ cuerpos). De este modo, debemos considerar soluciones aproximadas mediante métodos numéricos.

La solución numérica a un problema de valor inicial no se obtiene como una aproximación continua de la solución exacta, sino como un conjunto de aproximaciones de los valores exactos sobre ciertos valores discretos de la variable independiente, en nuestro caso, el tiempo $t$. En el planteo más simple, la solución numérica es construida a partir de la condición inicial sobre un conjunto de intervalos temporales equiespaciados de paso $\tau$ constante.

En la literatura matemática existen numerosos métodos numéricos para resolver un problema de valor inicial. Tales métodos comprenden al método de Taylor, los métodos de Runge-Kutta y sus derivados, métodos de extrapolación como el de Bulirsch-Stoer y métodos multipasos como los de Adams-Bashforth o Adams-Moulton. Estos métodos, si bien son precisos y apropiados en muchas circunstancias, no respetan, por otra parte, la naturaleza física del sistema que originó el conjunto de ecuaciones diferenciales. En particular, tales métodos introducen una componente espuria en el cálculo de la energía del sistema de manera tal que la misma no se mantiene constante (y ni siquiera acotada) como sería de esperarse en un sistema conservativo, sino que por el contrario, su magnitud varía monótonamente con el tiempo (Kinoshita y otros, 1990). Es claro, pues, que para estudiar el comportamiento dinámico de un sistema físico sobre un rango considerable de tiempo, es necesario disponer de integradores numéricos que respeten la física del problema. Tales métodos son los integradores simplécticos.

Un método de integración de las ecuaciones de movimiento de Hamilton de un sistema se dice que es simpléctico si las aproximaciones obtenidas de las variables canónicas en un instante de tiempo y las correspondientes a un instante posterior están relacionadas por una transformación canónica. De este modo, la transformación canónica que es solución exacta del problema es aproximada por otra transformación canónica próxima en un sentido a precisar. El hecho de que las soluciones numéricas estén relacionadas por una transformación canónica permiten asegurar que:

\footnotetext{
${ }^{1}$ El apéndice A presenta una discusión básica de los elementos de la formulación hamiltoniana.
} 
I. Un integrador simpléctico preserva la estructura del espacio de fases del sistema.

II. La acumulación de los errores de truncamiento no introduce una componente secular en el cómputo de la energía del sistema, por lo que la variación de la misma se mantiene acotada.

Nótese, sin embargo, que la deseable propiedad de mantener acotada la energía es satisfecha en tanto, y en cuanto, el paso de integración se mantenga constante. Si el paso de integración es variado durante la integración, no es posible asegurar que los errores en el cálculo de la energía del sistema se mantengan acotados (Yoshida, 1993).

El procedimiento para construir un integrador simpléctico consiste esencialmente en reescribir el hamiltoniano $H$ del sistema como la suma de un conjunto de partes, cada una de las cuales puede ser resuelta por su cuenta. Entonces, para un dado instante de tiempo, tales soluciones son combinadas de manera tal que aproximen a la solución del problema en dicho instante. Un formalismo apropiado para generar integradores simplécticos a partir de tal división del hamiltoniano, se basa en la formulación hamiltoniana de la mecánica en términos de los corchetes de Poisson y las series de Lie.

En dicho formalismo, la evolución temporal en el espacio de las fases de una función $u=u(q, p)$ de las variables canónicas $q, p$, sobre un intervalo temporal $\tau$ está dada por

$$
u(t)=e^{t \hat{\mathrm{H}}} u(0),
$$

siendo $\hat{\mathrm{H}}$ el operador diferencial lineal

$$
\hat{\mathrm{H}} \equiv[, H]
$$

donde los corchetes denotan el corchete de Poisson. Suponiendo que dividimos el hamiltoniano $H$ en dos partes

$$
H=H_{0}+H_{1},
$$

la evolución temporal de $u$ es

$$
u(\tau)=e^{\tau\left(\hat{\mathrm{H}}_{0}+\hat{\mathrm{H}}_{1}\right)} u(0) .
$$

Si el operador exponencial de esta ecuación es aproximado por la composición de los operadores exponenciales de los hamiltonianos por separado, esto es,

$$
e^{\tau\left(\hat{\mathrm{H}}_{0}+\hat{\mathrm{H}}_{1}\right)} \approx e^{\tau \hat{\mathrm{H}}_{1} / 2} e^{\tau \hat{\mathrm{H}}_{0}} e^{\tau \hat{\mathrm{H}}_{1} / 2},
$$

resulta que tal aproximación tiene un error de truncamiento proporcional a $\tau^{2}$. Esto conduce, pues, a un integrador simpléctico de segundo orden dado por

$$
u(\tau)=e^{\tau \hat{\mathrm{H}}_{1} / 2} e^{\tau \hat{\mathrm{H}}_{0}} e^{\tau \hat{\mathrm{H}}_{1} / 2} u(0) .
$$

La aplicación de cada operador exponencial en esta ecuación es equivalente a resolver las ecuaciones de movimiento considerando presente solamente la correspondiente parte del hamiltoniano. En consecuencia, cada paso del integrador consiste en tres subpasos:

I. El sistema se avanza sujeto sólo a la acción de $H_{1}$ sobre la mitad del paso de integración. 
II. A continuación se avanza sobre el intervalo temporal completo bajo la acción de $H_{0}$. III. Finalmente se avanza otra mitad del paso temporal bajo la acción de $H_{1}$.

En este caso, puede mostrarse (Yoshida, 1993) que el integrador resuelve en forma exacta un hamiltoniano $\tilde{H}=H+H_{\text {err }}$ donde

$$
H_{\text {err }}=\frac{\tau^{2}}{12}\left[\left[H_{1}, H_{0}\right], H_{0}+\frac{1}{2} H_{1}\right]+\text { términos proporcionales a } \tau^{4},
$$

para $\tau$ suficientemente pequeño.

Consideremos ahora, específicamente, el problema de $N$-cuerpos. El hamiltoniano de un sistema de $n=N+1$ partículas de masas $m_{i}(i=0, \ldots, N)$ sujetas únicamente a la acción de sus mutuas atracciones gravitatorias está dado por

$$
H(\mathbf{q}, \mathbf{p})=\sum_{i=0}^{N} \frac{\left|\mathbf{p}_{i}\right|^{2}}{2 m_{i}}-G \sum_{i=0}^{N-1} \sum_{j=i+1}^{N} \frac{m_{i} m_{j}}{\left|\mathbf{q}_{i}-\mathbf{q}_{j}\right|},
$$

donde $\mathbf{q}_{i}=\left(x_{i}, y_{i}, z_{i}\right)$ y $\mathbf{p}_{i}=\left(p_{x_{i}}, p_{y_{i}}, p_{z_{i}}\right)$ son los vectores posición y momento del cuerpo $i$-ésimo (de masa $m_{i}$ ) respecto a un sistema de referencia inercial de coordenadas cartesianas y $G$ es la constante de gravitación universal. La división más simple de este hamiltoniano consiste en separarlo en el término correspondiente a la energía cinética y el correspondiente a la energía potencial:

$$
H=K(\mathbf{p})+U(\mathbf{q})
$$

En tal caso, el integrador de segundo orden dado por el procedimiento prescrito por la ecuación (2-6), calcula la evolución del sistema, desde un estado inicial, sobre un paso de integración $\tau$ según:

$$
\begin{aligned}
\tilde{\mathbf{p}} & =\mathbf{p}(0)-\frac{\tau}{2} \frac{\partial U}{\partial \mathbf{q}}(\mathbf{q}(0)), \\
\mathbf{q}(\tau) & =\mathbf{q}(0)+\tau \frac{\partial K}{\partial \mathbf{p}}(\tilde{\mathbf{p}}), \\
\mathbf{p}(\tau) & =\tilde{\mathbf{p}}-\frac{\tau}{2} \frac{\partial U}{\partial \mathbf{q}}(\mathbf{q}(\tau)) .
\end{aligned}
$$

Estas ecuaciones definen el bien conocido integrador simpléctico de segundo orden denominado leap-frog.

Sin embargo, como ya hemos notado, para un disco kepleriano existe un cuerpo central cuya masa $m_{0}$ es mucho mayor que la de los restantes cuerpos $m_{i}(i \neq 0)$. De este modo, la fuerza gravitatoria del cuerpo central es usualmente la fuerza dominante y resulta más útil escribir el hamiltoniano del sistema como

$$
H=H_{\mathrm{Kep}}+H_{\mathrm{int}}
$$

donde $H_{\text {Kep }}$ es la parte del hamiltoniano que describe el movimiento central de $N$ cuerpos en torno al cuerpo $m_{0}$, en tanto $H_{\text {int }}$ es la parte que describe la interacción de los restantes cuerpos unos con otros. Así, el hamiltoniano es separado en una parte integrable junto con otra parte que es considerada como una perturbación. Los integradores 
simplécticos basados en tal división del hamiltoniano son conocidos como integradores simplécticos de variables mixtas (integradores MVS, por mixed variable symplectic methods).

La manera precisa en que tal división del hamiltoniano es realizada depende de las coordenadas escogidas. Los métodos MVS estándar utilizan coordenadas jacobianas ${ }^{2}$. Un ampliamente difundido método MSV de segundo orden es el integrador SWIFT de Levison y Duncan (1994). Sin embargo, resulta más adecuado (y computacionalmente más simple) utilizar las denominadas variables democráticas heliocéntricas. Tales variables consisten en coordenadas $\mathbf{Q}$ respecto al cuerpo central y momentos $\mathbf{P}$ respecto al baricentro del sistema. Las mismas constituyen también variables canónicas ${ }^{3}$ y, en términos de ellas, el hamiltoniano es escrito como

$$
H(\mathbf{Q}, \mathbf{P})=H_{\mathrm{Kep}}+H_{\mathrm{int}}+H_{\odot},
$$

donde

$$
\begin{aligned}
H_{\mathrm{Kep}} & =\sum_{i=1}^{N}\left(\frac{\left|\mathbf{P}_{i}\right|^{2}}{2 m_{i}}-\frac{G m_{i} m_{0}}{\left|\mathbf{Q}_{i}\right|}\right), \\
H_{\mathrm{int}} & =-G \sum_{i=1}^{N-1} \sum_{j=i+1}^{N} \frac{m_{i} m_{j}}{\left|\mathbf{Q}_{i}-\mathbf{Q}_{j}\right|}, \\
H_{\odot} & =\frac{1}{2 m_{0}}\left|\sum_{i=1}^{N} \mathbf{P}_{i}\right|^{2}
\end{aligned}
$$

Aquí un tercer término $H_{\odot}$ aparece en el hamiltoniano debido al momento baricéntrico del cuerpo central. Para una división del hamiltoniano en tres términos, el integrador simpléctico de segundo orden (2-6) puede ser fácilmente extendido. Ahora, un paso de integración toma la forma:

$$
u(\tau)=e^{\tau \hat{\mathrm{H}}_{\mathrm{int}} / 2} e^{\tau \hat{\mathrm{H}}_{\odot} / 2} e^{\tau \hat{\mathrm{H}}_{\mathrm{Kep}}} e^{\tau \hat{\mathrm{H}}_{\odot} / 2} e^{\tau \hat{\mathrm{H}}_{\mathrm{int}} / 2} u(0) .
$$

Analizando como actúa cada parte del hamiltoniano sobre las variables canónicas vemos que el método de integración procede como sigue:

I. Las coordenadas permanecen fijas y cada cuerpo recibe una aceleración proveniente de los otros cuerpos (pero no del cuerpo principal) que modifica su momento sobre un intervalo temporal $\tau / 2$.

II. Los momentos permanecen fijos, y cada cuerpo sufre un desplazamiento en su posición en la cantidad $\left(\tau / 2 m_{0}\right) \sum_{i=1}^{N} \mathbf{P}_{i}$.

III. Cada cuerpo evoluciona en torno a una órbita kepleriana (con la misma localización central y la misma masa central) en un tiempo $\tau$.

IV. Como en el paso II.

V. Como en el paso I.

\footnotetext{
${ }^{2}$ Las coordenadas jacobianas constituyen un sistema de referencia donde la posición y momento de cada cuerpo es tomada con respecto al centro de masa de todos los cuerpos con índices menores respecto a cierto orden asignado de antemano.

${ }^{3}$ Ver apéndice B para una demostración.
} 


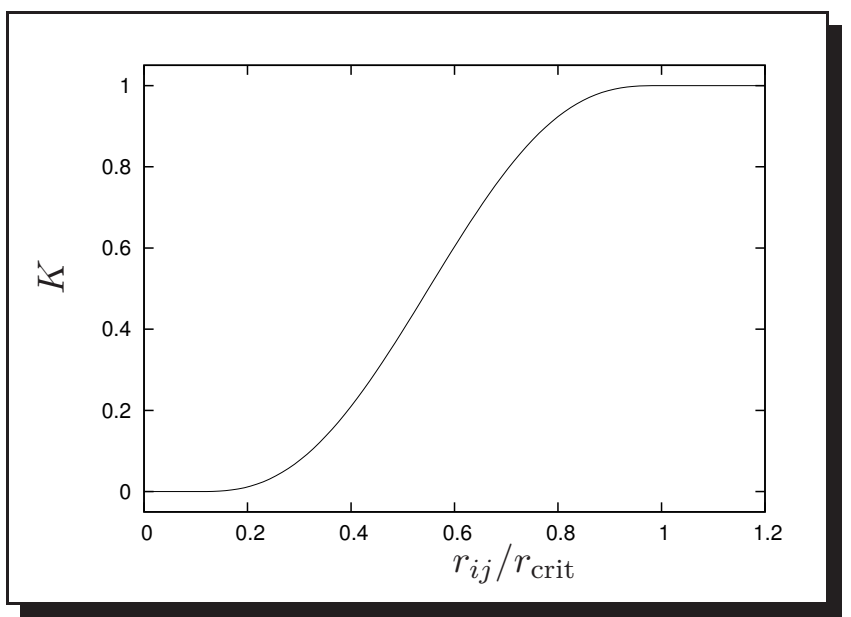

Figura 2.1. Una función de encuentro $K$ apropiada para el algoritmo de integración híbrido en función de la separación, $r_{i j}=\left|\mathbf{Q}_{i}-\mathbf{Q}_{j}\right|$, de dos partículas.

Este esquema de integración tiene dos claras ventajas respecto al método leap-frog. En primer lugar el movimiento kepleriano dado por la acción de $H_{\text {Kep }}$ tiene una solución analítica bien conocida. Por lo tanto, el avance de cada partícula del sistema sobre un dado intervalo temporal es efectuado con un esfuerzo computacional mínimo. Más aún, distinto de los métodos MSV estándar, todos los cuerpos orbitan alrededor del mismo cuerpo central, con la misma masa central. Por otra parte, supuesto que todos los cuerpos permanecen alejados unos de otros,

$$
\left|H_{\text {int }}\right| \ll\left|H_{\text {Kep }}\right|, \quad\left|H_{\odot}\right| \ll\left|H_{\mathrm{Kep}}\right|
$$

por la razón de la masa de los cuerpos a la del cuerpo central, $\epsilon=\sum_{i=1}^{N} m_{i} / m_{0}$. En consecuencia, cada paso del integrador tiene un error de truncamiento de proporcional a $\epsilon \tau^{2}$, en vez de a $\tau^{2}$ como en el integrador leapfrog. Así, para la misma precisión, podemos tomar un paso temporal $\epsilon^{-1 / 2}$ veces el paso del método leapfrog, lo cual redunda en un menor tiempo de cómputo (Kinoshita y otros, 1991; Duncan y otros, 1998).

Por supuesto, lo dicho es válido en tanto no se produzcan encuentros próximos entre los cuerpos. Si dos cuerpos se aproximan lo suficiente, la distancia mutua entre ellos es pequeña y, en consecuencia, el correspondiente término en $H_{\text {int }}$ resulta comparable en tamaño, o incluso es mayor, a $H_{\text {Kep }}$. Debido a esto, el error de truncamiento por paso se incrementa de $\epsilon \tau^{2}$ a $\tau^{2}$. Para mantener la precisión, los integradores tradicionales reducen el tamaño del paso de integración. Sin embargo, como ya hemos indicado, en un integrador simpléctico un cambio del paso destruye sus propiedades simplécticas.

\subsection{Resolviendo encuentros.}

Diseñar un integrador simpléctico que detecte e integre en forma precisa los encuentros no es una tarea fácil. Los métodos MSV estándar al utilizar coordenadas jacobianas no pueden seguir apropiadamente los encuentros. Una solución es dada por Duncan y otros (1998) en su integrador SYMBA, un integrador simpléctico de paso múltiple que 
utiliza coordenadas heliocéntricas democráticas donde el término perturbativo es subdividido de manera tal que a cada parte se le asigna un distinto (pero fijo) paso de integración, donde los términos más débiles reciben los más grandes pasos.

Una solución alternativa consiste en reescribir la división del hamiltoniano de forma tal que $H_{\text {int }}$ sea nuevamente pequeño frente a $H_{\mathrm{Kep}}$. Una manera simple de proceder consiste en transferir el término conflictivo de $H_{\text {int }}$ a $H_{\text {Kep }}$ mientras dura el encuentro. Sin embargo, este intercambio a lo largo de los sucesivos encuentros termina destruyendo la naturaleza simpléctica del integrador.

Para lograr un integrador realmente simpléctico, ningún término tiene que ser transferido entre las diversas partes del hamiltoniano. Un procedimiento adecuado es descripto por Chambers (1999), quien reescribe los términos de $H_{\text {Kep }}$ y $H_{\text {int }}$ introduciendo una función de encuentro $K$ :

$$
\begin{aligned}
H_{\mathrm{Kep}} & =\sum_{i=1}^{N}\left(\frac{\left|\mathbf{P}_{i}\right|^{2}}{2 m_{i}}-\frac{G m_{i} m_{0}}{\left|\mathbf{Q}_{i}\right|}\right)-G \sum_{i=1}^{N-1} \sum_{j=i+1}^{N} \frac{m_{i} m_{j}}{\left|\mathbf{Q}_{i}-\mathbf{Q}_{j}\right|}\left[1-K\left(\left|\mathbf{Q}_{i}-\mathbf{Q}_{j}\right|\right)\right], \\
H_{\text {int }} & =-G \sum_{i=1}^{N-1} \sum_{j=i+1}^{N} \frac{m_{i} m_{j}}{\left|\mathbf{Q}_{i}-\mathbf{Q}_{j}\right|} K\left(\left|\mathbf{Q}_{i}-\mathbf{Q}_{j}\right|\right) .
\end{aligned}
$$

La forma de $K$ es tal que tiende a la unidad cuando la separación $\left|\mathbf{Q}_{i}-\mathbf{Q}_{j}\right|$ entre dos cuerpos $i$ y $j$ es grande, mientras que tiende a cero cuando tal separación es pequeña, tal como se ilustra en la Figura 2.1. Esto asegura que $H_{\text {int }}$ permanezca pequeño frente a $H_{\text {Kep }}$ aún durante un encuentro. En ausencia de encuentros $H_{\text {Kep }}$ puede ser integrado analíticamente. Sin embargo, cuando dos cuerpos tienen un encuentro tenemos un problema de tres cuerpos (el cuerpo central y los cuerpos en encuentro). Aunque esta situación ya no es integrable analíticamente, en la práctica el problema puede ser integrado numéricamente con un integrador tradicional (típicamente utilizando el método de Bulirsch-Stoer) al grado de precisión necesaria. La incorporación de un integrador no simpléctico dentro del esquema simpléctico anterior confiere al integrador resultante la naturaleza de híbrido. Su procedimiento es entonces, como sigue:

I. Las coordenadas permanecen fijas y cada cuerpo recibe una aceleración proveniente de los otros cuerpos (pero no del cuerpo principal) pesada en las situaciones de encuentros por un factor $K$, que modifica su impulso sobre un intervalo temporal $\tau / 2$.

II. Los impulsos permanecen fijos, y cada cuerpo sufre un desplazamiento en su posición en la cantidad $\left(\tau / 2 m_{0}\right) \sum_{i=1}^{N} \mathbf{P}_{i}$.

III. Los cuerpos que no están en encuentros se mueven en una órbita kepleriana en torno al cuerpo principal sobre un intervalo temporal $\tau$, en tanto que para los cuerpos que están en encuentros binarios, los términos keplerianos y los términos de encuentro, pesados por $(1-K)$, son integrados numéricamente un paso $\tau$.

IV. Como en el paso II.

V. Como en el paso I.

La implementación de Chambers de tal integrador simpléctico híbrido es incluida en su paquete denominado MERCURY (Chambers y Migliorini, 1997). 


\subsection{El algoritmo de árbol jerárquico octal.}

El esquema de integración de Chambers satisface los tres primeros requisitos enunciados al comienzo de este capítulo. A saber, es un integrador simpléctico (híbrido) adecuado para tratar la dinámica de un disco kepleriano, y capaz de resolver encuentros. Sin embargo, al igual que en los otros códigos mencionados, la interacción entre partículas es evaluada directamente. Este procedimiento partícula-partícula demanda mucho tiempo de cómputo. En efecto, el cómputo de la interacción requiere de un total de operaciones proporcional al cuadrado del número de partículas, puesto que, para $N$ partículas, hay $\frac{1}{2} N(N-1)$ interacciones a evaluar. Este procedimiento es razonable para sistemas que involucran unos pocos de cientos de partículas, pero como el tiempo de cómputo se incrementa tan rápidamente con el número de partículas, simulaciones numéricas con miles de partículas resultan computacionalmente costosas y aquellas con millones de partículas resultan inalcanzables. Es claro, pues, que para tales sistemas debe considerarse un procedimiento alternativo al cálculo directo.

Una manera fructífera de disminuir el tiempo de cómputo en la evaluación de la interacción gravitacional entre partículas consiste en implementar el llamado algoritmo de árbol jerárquico octal, puesto que para este método el número total de operaciones involucradas en el cómputo de las interacciones resulta proporcional a $N \log N$ (Pfalzner y Gibbon, 1999). El algoritmo explota el hecho de que el potencial gravitatorio decae con la inversa de la distancia, con lo cual las partículas interactúan fuertemente con sus vecinos más próximos, pero menos información acerca de la distribución de masa resulta necesaria para describir sus interacciones con partículas más distantes. Así mientras la interacción sobre una partícula debida a otras partículas cercanas es evaluada en forma directa como una interacción partícula-partícula, la interacción debida a las partículas más distantes puede considerarse como una interacción directa entre la partícula y dicho conjunto de partículas distantes, representado como una partícula cuya masa es la total del grupo colocada en el centro de masa del mismo. Por supuesto, esto conduce a una pérdida de información acerca de la distribución espacial de masa dentro del grupo, lo cual, a su vez, introduce un error en el cómputo de la interacción. Sin embargo incluyendo los momentos multipolares del grupo, esta información puede ser recuperada en parte, obteniéndose así una precisión suficiente. Para llevar a cabo esta idea en forma eficiente, el algoritmo construye una estructura jerárquica que recuerda a un árbol (de aquí el nombre) a partir de una división particular del espacio. Esta estructura de árbol provee una manera sistemática de determinar el grado de "cercanía" entre dos diferentes partículas sin calcular explícitamente la distancia entre cada par de partículas y permite calcular eficientemente los momentos multipolares.

Un código de árbol adecuadamente adaptado para la dinámica de planetesimales es presentado por Brunini y Viturro (2003). Tal código, sin embargo, al integrar las ecuaciones de movimiento con el algoritmo leapfrog, no toma ventaja del hecho de que las partículas evolucionan principalmente sujetos a un potencial cental. Nuestro código, por otra parte, es una escritura desde cero del esquema de Chambers pero utilizando el código de árbol para el cómputo de las interacciones entre partículas contenidas en $H_{\text {int }}$. De este modo esperamos obtener la mejor performance. El código de árbol implementado es una modificación del código de Brunini y Viturro, el cual, a su vez se basa en el algoritmo básico descrito por Barnes y Hut (1986, 1989), Hernquist 
(1987), Barnes (1995) y Pfalzner y Gibbon (1999). Una somera descripción es dada a continuación. Los detalles de la implementación son descritos en Santamaría (2004).

El primer paso de un código de árbol consiste en la construcción del mismo. Dicha construcción comienza creando un cubo que contenga a todo el sistema e identificando el mismo con la celda más grande del árbol (conocida como raíz). Luego, esta celda es subdividida en ocho subceldas de igual tamaño y, a su vez, cada una de ellas es dividida recursivamente hasta que las celdas finales (las hojas del árbol) contienen uno o ningún cuerpo. Con esta división espacial del sistema, las masas totales, centros de masa y momentos cuadrupolares de cada celda son calculados recorriendo el árbol en forma descendente utilizando fórmulas recursivas para los cálculos. Esto provee un desarrollo a segundo orden del potencial gravitatorio del sistema que es utilizado para computar la interacción del sistema sobre un cuerpo. Para ello se comienza recorriendo el árbol desde la raíz hacia las hojas. Una celda contribuirá como un todo a esta interacción si se encuentra suficientemente lejos del cuerpo. El criterio para decidir cuando debe o no subdividirse está relacionado con el denominado parámetro de expansión $\theta$ del árbol, definido como la razón entre el tamaño de la celda y su distancia al cuerpo bajo consideración. En la situación más simple la celda es subdividida cuando el parámetro de expansión es mayor que cierto valor límite. Nótese que la situación extrema $\theta=0$ es equivalente a calcular todas las interacciones en forma directa, partícula a partícula, requiriendo pues un esfuerzo de cómputo proporcional a $N^{2}$, mientras que la otra situación extrema, $\theta \rightarrow \infty$, produce una muy baja resolución espacial, con la interacción calculada solamente entre partículas y grandes grupos de partículas, lo cual, aunque es muy rápido, es extremadamente impreciso. En la práctica, para sistemas sin contornos impuestos, valores de $\theta$ en el rango $0.5-0.7$ han demostrado ser adecuados (Pfalzner y Gibbon, 1999).

\subsection{Detectando encuentros y colisiones.}

La estructura de árbol puede ser utilizada para determinar las partículas que tienen encuentros. El código requiere conocer al comienzo de un paso de integración qué partículas están en encuentros. Una condición de encuentro puede ser definida como sigue. Para cada partícula $i$, computamos su radio de Hill, definido como

$$
R_{\mathrm{H}}=r_{i}\left(\frac{m_{i}}{3 m_{0}}\right)^{1 / 3}
$$

donde $r_{i}$ es la distancia heliocéntrica de la partícula y $m_{i}$ su masa. Entonces para un cuerpo $i$, las partículas $j$ en encuentro serán aquellos vecinos más próximos cuyas distancias a $i$ satisfagan la condición

$$
r_{i j}<\operatorname{máx}\left(\alpha R_{\mathrm{H}_{i}}, \alpha R_{\mathrm{H}_{j}}\right)
$$

siendo $\alpha$ un factor de proporcionalidad, cuyo valor, adoptado por la mayoría de los autores, es 3 (Levison y Duncan, 1994; Duncan y otros, 1998; Chambers, 1999; Brunini y Melita, 2002). 
Determinar las partículas en encuentros requiere así de un algoritmo para la búsqueda de vecinos más próximos. Un procedimiento directo basado en evaluar la distancia mutua entre cada par de objetos demanda claramente un costo computacional que crece con $N^{2}$. Un algoritmo más eficiente puede ser construido en base a la estructura de árbol. El procedimiento, en esencia, es como sigue. Nuevamente los detalles de la implementación pueden verse en Santamaría (2004).

Para la búsqueda de los vecinos próximos de un cuerpo dado dentro de una distancia $h$, el cuerpo es encerrado en un cubo de lados $2 h$. Comenzando con la celda raíz, el árbol es entonces recorrido de manera análoga al procedimiento del cómputo de las interacciones. La diferencia es que, ahora, el criterio de apertura es reemplazado por la cuestión de si el cubo de búsqueda se traslapa con el cubo de la celda examinada. Si tal traslapo no ocurre, entonces la rama del árbol bajo tal celda no contiene vecinos próximos y la búsqueda sobre la misma es detenida. Si, por el contrario, el traslapo ocurre, la celda es dividida en sus subceldas y la búsqueda continúa sobre el siguiente nivel de jerarquía de tal rama. Si la celda que se traslapa con el cubo de búsqueda es una hoja (esto es, es un cuerpo) se verifica si tal partícula yace efectivamente dentro de la distancia $h$. Si este es el caso, tal cuerpo es agregado a la lista de vecinos próximos. Este procedimiento es efectuado sobre todas las ramas del árbol.

Por otra parte, si las partículas del sistema son, en realidad, cuerpos de radio finito, durante un encuentro es posible que el par de cuerpos involucrados sufran una colisión. Como es usual en las simulaciones de $N$-cuerpos, la condición de colisión es que la distancia mutua entre los dos cuerpos en encuentro sea menor o igual que la suma de sus radios. El tratamiento más simple de la colisión es suponer que la misma es perfectamente plástica. Esto es, los dos cuerpos se fusionan sin fragmentarse, originando un nuevo cuerpo cuya masa es igual a la suma de las masas del par y cuya posición y velocidad, inmediatamente después de la colisión, es la del centro de masa del par inmediatamente antes de la colisión. En tales circunstancias hay una disminución en la energía cinética, con lo que la colisión perfectamente plástica es una colisión inelástica de primera clase (o endoérgica). Es claro que, debido a las colisiones, la energía total del sistema no se conservará.

\subsection{Desempeño del código.}

Con el fin de evaluar el desempeño del código hemos realizado una serie de experimentos numéricos, los cuales son esencialmente los presentados en Brunini y Viturro (2003). Los resultados obtenidos están en excelente acuerdo con los experimentos anteriores, aun utilizando un paso temporal considerablemente mayor. Esto es debido a la naturaleza híbrida del código, el cual aprovecha el hecho de que el movimiento ocurre bajo un potencial perturbado del potencial central kepleriano. Nuevamente, en Santamaría (2004) puede verse una discusión completa de los experimentos numéricos; aquí solo presentaremos los resultados más relevantes.

El escenario considerado corresponde a las simulaciones numéricas de Kokubo e Ida (1996) de la etapa de crecimiento en fuga de la acreción de planetesimales. Como hemos mencionado en el capítulo introductorio, las simulaciones numéricas muestran que una 


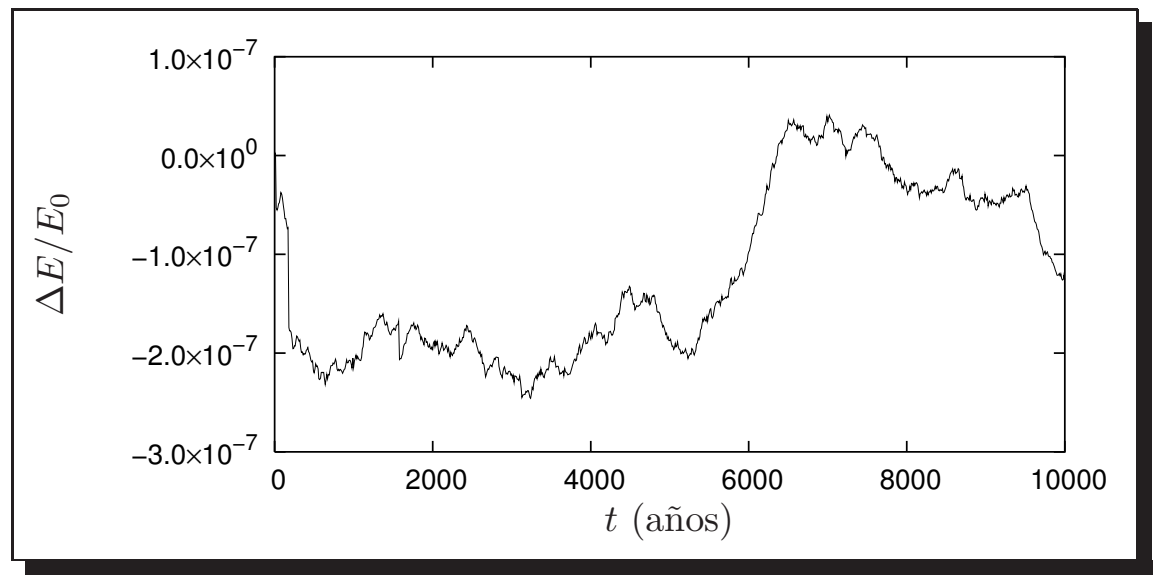

Figura 2.2. Error relativo en la energía total del sistema en función del tiempo.

población de planetesimales a 1 UA del cuerpo central y sobre una estrecha franja en semieje mayor, interactúan de manera tal que un cuerpo crece mucho más rápidamente separándose del resto de la población y convirtiéndose en un embrión planetario con un tamaño en el rango entre Mercurio y Marte en una escala de tiempo de unos $10^{5}$ años.

En nuestro caso, la simulación consiste inicialmente en $N=2000$ planetesimales de igual masa, $m=10^{24} \mathrm{~g}$ (con densidades $\rho=2 \mathrm{~g} \mathrm{~cm}^{-3}$ ) distribuidos uniformemente en una región anular alrededor de $a=1$ UA con un ancho $\Delta a=0.2$ UA. Las excentricidades e inclinaciones de las órbitas osculatrices iniciales de los planetesimales están dadas por distribuciones gaussianas con dispersiones $\left\langle e^{2}\right\rangle^{1 / 2}=2\left\langle i^{2}\right\rangle^{1 / 2}=4 R_{\mathrm{H}} / a$, siendo $R_{\mathrm{H}}$ el radio de Hill de los cuerpos.

En el primer conjunto de experimentos numéricos, los cuerpos fueron tratados como masas puntuales, con lo cual las colisiones fueron ignoradas. Esto nos permitió testear la precisión global del código (medida por la conservación de la energía total del sistema), la precisión del código de árbol en el cómputo de las interacciones entre los planetesimales, y la eficiencia en la detección de encuentros. Un análisis de las simulaciones mostró que la elección de un parámetro de expansión $\theta=0.7$ y un paso de integración $\tau=0.005$ años constituyen una apropiada elección para satisfacer los requisitos anteriores. En particular, como se ilustra en la Figura 2.2, el error relativo en la energía total del sistema no muestra un comportamiento secular a lo largo de 10000 años. Aun cuando las variaciones de la energía sobre períodos cortos de tiempo son relativamente grandes, la dispersión de los valores respecto de la media es $8.7 \times 10^{-8}$.

En el segundo conjunto de simulaciones se permitió la posibilidad de colisiones entre planetesimales. En estas circunstancias, tal como lo hicieran Kokubo e Ida (1996), el radio inicial de los planetesimales fue aumentado en un factor 5 respecto del valor realista que se obtiene suponiendo que la densidad de los mismos es $\rho=2 \mathrm{~g} \mathrm{~cm}^{-3}$. Como muestran Kokubo e Ida, esto solo cambia la escala de tiempo de acreción, pero no el modo de crecimiento de los planetesimales. Con las mismas condiciones iniciales que antes, el sistema fue integrado hasta $t=20000$ años con un paso de integración $\tau=0.01$ años (tomando el parámetro de expansión del árbol $\theta=0.7$ ). Los resultados obtenidos son ilustrados en el conjunto de Figuras 2.3, 2.4 y 2.5, las cuales deberían 


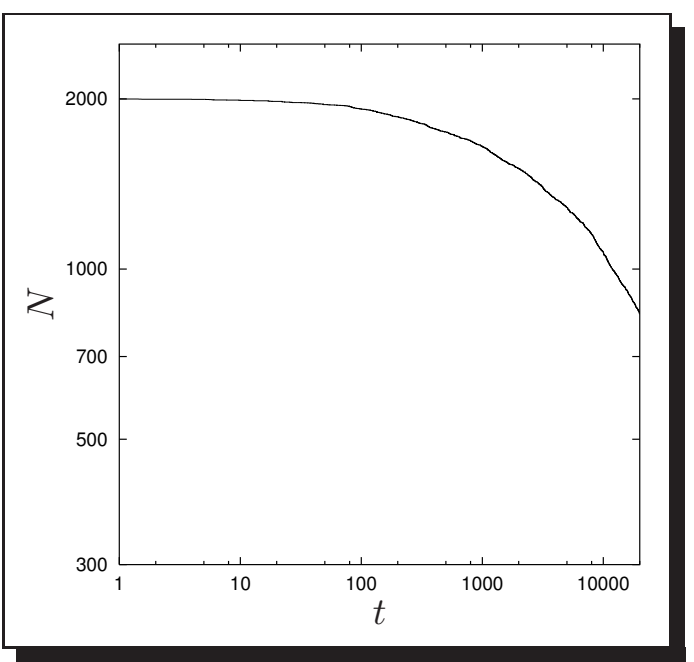

Figura 2.3. Número de cuerpos en función del tiempo. Abscisas: tiempo en años, ordenadas: número de cuerpos (escala logarítmica sobre ambos ejes).

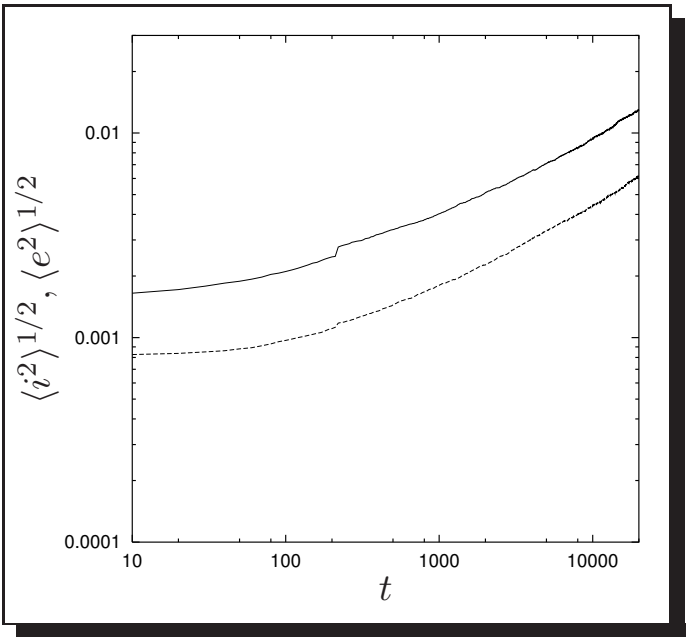

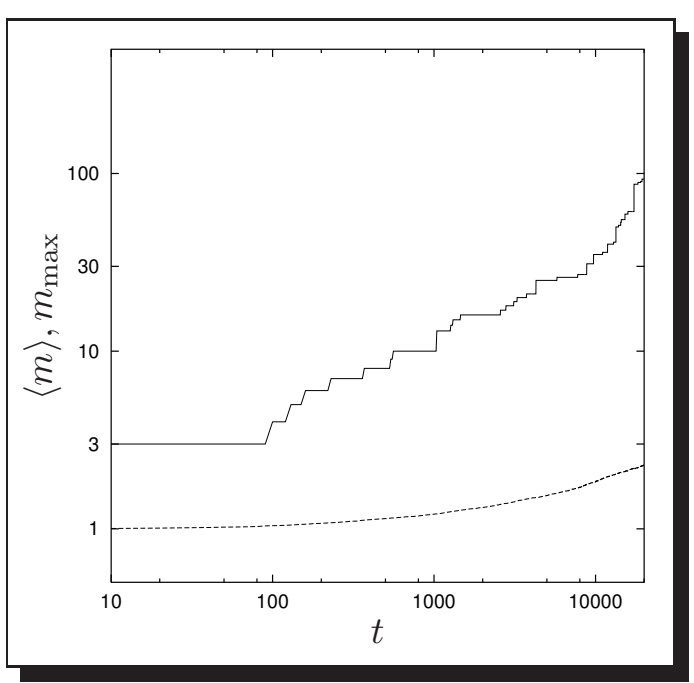

Figura 2.4. Masa máxima en la simulación (curva de trazo continuo) y masa media (curva de trazo punteado) en función del tiempo. Abscisas: tiempo en años, ordenadas: masa en $10^{24} \mathrm{gr}$ (escala logarítmica sobre ambos ejes).

Figura 2.5. Evolución temporal de la media cuadrática de la excentricidad (curva de trazo continuo) y de la inclinación (curva de trazo punteado). Abscisas: tiempo en años (escala logarítmica sobre ambos ejes). 
compararse con las correspondientes al trabajo de Kokubo e Ida. La Figura 2.3 muestra la evolución del número total de cuerpos en función del tiempo. Este decrece de 2000 a 833 en los 20000 años (en la simulación de Kokubo e Ida, el número final de cuerpos es 715). La Figura 2.4 muestra la evolución temporal de la mayor masa en cada instante de tiempo y la masa media de los planetesimales (exceptuando la masa máxima). Al final de la integración, la masa del cuerpo más masivo es unas 93 veces el valor de su masa inicial. Este valor difiere en un factor 2 del resultado de Kokubo e Ida, la cual es atribuible a un evento colisional aleatorio al final de la simulación. De cualquier manera, la razón de la masa máxima a la masa media crece monótonamente con el tiempo a una tasa similar a la obtenida por Kokubo e Ida. La Figura 2.5 muestra la evolución temporal de las medias cuadráticas de la excentricidad e inclinación de las órbitas osculatrices de los planetesimales. Ambas se incrementan monótonamente con el tiempo y puede apreciarse que su razón se mantiene aproximadamente en 2, el valor inicial.

\subsection{Modificaciones al código para problemas de in- teracción planeta-disco.}

Todo escenario de la interacción planeta-disco requiere distinguir entre cuerpos planetarios y planetesimales. Mientras que estos últimos conforman el disco de partículas, los primeros constituyen objetos embebidos en el disco con masas varios órdenes de magnitud mayores. Como muestran Ida y Makino (1993), aun para un pequeño planeta, la interacción entre los componentes del sistema (planeta-planetesimal y planetesimalplanetesimal) evoluciona hacia un estado dominado por el planeta, esto es, por la interacción planeta-planetesimal, convirtiéndose el planeta en un fuerte perturbador del disco. En estas circunstancias la interacción entre planetesimales, la autointeracción del disco, puede ser despreciada.

Ahora bien, el código original desarrollado inicialmente en el contexto de la tesis de licenciatura (Santamaría, 2004) trata a todos los cuerpos que orbitan a la estrella central en pie de igualdad frente a las interacciones mutuas. La posibilidad de distinguir entre planetas y planetesimales es una capacidad que se incorporó al código en el marco de esta tesis de doctorado. Con tal distinción podemos considerar ahora que, en la evaluación de la interacción entre cuerpos, los planetas interactúan entre sí y con los planetesimales, pero los planetesimales del disco no interactúan gravitatoriamente entre sí. Específicamente, para un planeta la interacción gravitatoria de los restantes cuerpos (planetas y planetesimales) es evaluada a través del árbol, mientras que para cada planetesimal la interacción gravitatoria, dada únicamente con los planetas, es evaluada en forma directa. En el siguiente capítulo examinaremos diversos escenarios particulares de la interacción planeta-disco que resultaron útiles para poner a prueba el integrador desarrollado.

Disponibilidad del código. El código fuente del integrador DAEDALUS desarrollado se encuentra disponible, junto con instrucciones específicas para su compilación y uso, en http://gcp. fcaglp. unlp. edu .ar/integrantes : psantamaria:daedalus : start. 



\section{Capítulo 3}

\section{Escenarios de migración planetaria por interacción planeta-disco}

\subsection{Introducción.}

En este capítulo consideramos diversos escenarios de migración planetaria, presentes en la literatura, con el fin de poner a prueba la capacidad del código numérico expuesto en el capítulo anterior. Específicamente consideraremos la migración de un único cuerpo planetario embebido en un disco de planetesimales y dos escenarios de migración de los planetas gigantes del sistema solar, uno con la presencia de un disco de planetesimales masivo y, finalmente, el denominado modelo de Niza.

\subsection{Migración de un único cuerpo planetario.}

El primer escenario a abordar consiste en el estudio de la migración planetaria de un único cuerpo planetario sumergido en un disco de planetesimales de masa nebular mínima $\left(0.01 M_{\odot}\right)^{1}$ con densidad superficial constante $\left(\Sigma \sim 10 \mathrm{~g} \mathrm{~cm}^{-2}\right)$, prestando especial atención a su efecto sobre la estructura global del disco, tal como lo proponen y analizan Cionco y Brunini (2002). En dicho trabajo, la interacción planeta-disco es analizada desde una perspectiva resonante: a través de la determinación de la tasa de migración del planeta, medida en la fase en que la fricción dinámica domina la evolución orbital, se computa el torque ejercido por el disco. Los resultados obtenidos en una serie de simulaciones numéricas para planetas en el rango de una masa de Júpiter a diez masas terrestres (esto es, la masa de un núcleo de los planetas gigantes del Sistema Solar) son contrastados con los correspondientes a la formulación analítica de la fricción dinámica en discos de partículas (Lynden-Bell y Kalnajs, 1972) y, en virtud de la correspondencia destacada en un trabajo pionero de Goldreich y Tremaine (1979), con las expresiones analíticas de la teoría lineal de ondas de densidad para discos gaseosos bidimensionales. A continuación presentamos un breve resumen del marco teórico asociado con dichos trabajos.

\footnotetext{
${ }^{1}$ Ver apéndice $\mathrm{C}$ para una breve discusión del modelo de nebulosa solar de masa mínima.
} 
Consideremos un planeta inmerso en un disco de planetesimales, esto es, un cuerpo diferenciado del resto de las partículas del disco. En su movimiento el planeta ejerce fuerzas sobre el disco y la reacción a tales fuerzas influye asimismo en el movimiento del planeta. De este modo, podemos considerar que el planeta se mueve en un medio resistivo, donde la resistencia tiene su origen no en efectos viscosos como los producidos por el movimiento de un fluido, sino por efectos netamente gravitacionales. El concepto de tal fricción dinámica, como es denominada en la literatura, fue introducido por Chandrasekhar (1942) en el contexto de la dinámica estelar. En su formulación, el cuerpo masivo se mueve en un "mar" infinito de partículas y como resultado de los sucesivos encuentros gravitacionales el cuerpo masivo experimenta una continua desaceleración, efecto que resulta proporcional a la masa del cuerpo. Así, mientras la velocidad relativa del cuerpo respecto al medio disminuye, la velocidad relativa de las partículas se incrementa. El efecto neto es que la fricción dinámica tiende a llevar al sistema a un estado de equipartición de la energía donde las velocidades relativas de las partículas se incrementan a expensas de la disminución de la del cuerpo masivo. Aplicado al marco de la interacción planeta-disco, la fricción dinámica produce un decaimiento orbital del planeta. El consiguiente decrecimiento de su momento angular origina un aumento del momento angular de los planetesimales, aumentando así el grado de excitación del disco. El tiempo necesario para alcanzar el estado de equipartición de la energía, momento en el cual la fricción dinámica desaparece, se denomina tiempo de relajación. Nótese, sin embargo, que la aplicación de la formulación de Chandrasekhar de la fricción dinámica en discos keplerianos de planetesimales debe tener presente las limitaciones que la misma adolece en virtud de las hipótesis efectuadas en su deducción. A saber, ésta es derivada para un cuerpo moviéndose a través de un medio infinito y homogéneo de partículas y la interacción gravitacional es descripta en el marco del problema de los dos cuerpos. En un disco de planetesimales, donde los mismos se encuentran en órbitas keplerianas cuasi-circulares en torno a la estrella central, la velocidad relativa entre un cuerpo masivo y las partículas está determinada fundamentalmente por la cizalladura kepleriana establecida por la velocidad diferencial del disco (esto es, las partículas a distancias radiales menores que la del cuerpo masivo se mueven más rápidamente que el mismo, mientras que las más alejadas lo hacen más lentamente). Así pues, la descripción de la interacción gravitatoria requiere de la formulación del problema de tres cuerpos. Nótese, sin embargo, que la formulación de Chandrasekhar puede ser utilizada como una estimación de la fricción dinámica en un entorno local ${ }^{2}$. Pero, en el marco de la perspectiva resonante considerada en el trabajo de Cionco y Brunini discutido aquí, es necesario considerar una descripción más precisa. La formulación de la fricción dinámica en discos de partículas proviene de la dinámica estelar. Lynden-Bell y Kalnajs (1972) muestran que un perturbador embebido en un disco de partículas y rotando uniformemente produce torques sólo en ciertas posiciones resonantes, a saber, en las denominadas resonancia de corrotación y resonancias de Lindblad ${ }^{3}$. En términos generales, una resonancia aparece cuando una frecuencia característica del cuerpo perturbador coincide con una frecuencia del disco. Para el caso de un perturbador en una órbita circular con frecuencia angular $\Omega_{p}$ y un disco de frecuencia orbital $\Omega$, la

\footnotetext{
${ }^{2}$ Tal aproximación será utilizada, de hecho, en el capítulo siguiente.

${ }^{3}$ Ver el apéndice D para una deducción elemental del surgimiento de estas resonancias.
} 
resonancia de corrotación ocurre cuando ambas frecuencias coinciden

$$
\Omega=\Omega_{p},
$$

en tanto que una resonancia de Lindblad ocurre cuando

$$
m\left(\Omega-\Omega_{p}\right)= \pm \kappa_{0}
$$

donde $m$ es un entero y $\kappa_{0}$ es la frecuencia natural para oscilaciones radiales de las partículas del disco, denominada frecuencia epicíclica. Para un disco kepleriano, donde los pequeños apartamientos del valor $\Omega=\sqrt{G M_{\odot} / r^{3}}$ debido a la autointeracción son despreciados, hay, por un lado, una única resonancia de corrotación y ésta es coorbital con la localización del perturbador, y, por otro lado, reduciéndose $\kappa_{0}=\Omega$ (ver apéndice D), las localizaciones de las resonancias de Lindblad están dadas por

$$
r_{L}=\left(1 \pm \frac{1}{m}\right)^{2 / 3} r_{p}
$$

donde $r_{p}$ es el radio de la órbita circular del perturbador. Aquí el signo $+(-)$ corresponde a las resonancias de Lindblad externas (internas), las cuales resultan cada vez más próximas entre sí conforme nos acercamos a la localización del perturbador. Así pues, un planeta orbitando en el interior de un disco de planetesimales, intercambiará momento angular con el disco sólo en dichas resonancias (Lynden-Bell y Kalnajs, 1972). Por otra parte, para un disco de gas, la transferencia de momento angular en tales resonancias genera perturbaciones en su distribución de densidad, excitando así ondas de densidad las cuales transportan momento angular hacia zonas cada vez más alejadas del planeta. El torque neto, sin embargo, puede modelarse independientemente de los detalles de cómo estas ondas se propagan y disipan en el disco, tal como mostraron Goldreich y Tremaine $(1979,1980)$ (ver también Tanaka y otros, 2002 para una presentación y revisión mejorada). El cálculo, por cierto laborioso, procede considerando el perturbador sobre una órbita circular embebido en un disco de gas geométricamente delgado (de aquí la denominación de disco bidimensional). El potencial gravitatorio del perturbador es entonces descompuesto como una superposición de armónicos y, a continuación, se calcula, a partir de la linealización de las ecuaciones hidrodinámicas, la respuesta del gas a tales componentes en las localizaciones resonantes para así derivar el torque correspondiente. El punto fundamental del cálculo consiste en suponer que cuando la perturbación es lo suficientemente débil como para no alterar sustancialmente la densidad superficial del gas en el disco, el torque neto sobre el perturbador puede ser obtenido considerando la suma discreta de los torques ejercidos en cada resonancia. Nótese que, aunque este procedimiento da la magnitud del torque neto sobre el cuerpo, no da información sobre su signo. Ahora bien, el cuerpo gana momento angular a expensas del disco en las resonancias de Lindblad internas $\left(r_{L}<r_{p}\right)$ y por lo tanto tiende a moverse hacia afuera, en tanto que, en contrapartida, el gas tiende a moverse hacia adentro. La situación se revierte en las resonancias de Lindblad externas $\left(r_{L}>r_{p}\right)$ donde el cuerpo pierde momento angular y por lo tanto tiende a moverse hacia adentro, en tanto que el gas tiende a moverse hacia afuera. Nótese que, entonces, la interacción gravitatoria del cuerpo con el gas tiende a repeler el gas que se encuentra en las proximidades de la órbita del cuerpo. Ignorando por el momento la contribución de la resonancia de 
corrotación al torque, es claro que habrá cambios significativos en la órbita del cuerpo, esto es, una migración, si existe una diferencia sustancial en la magnitud de los torques opuestos originados en la resonancias internas y externas de Lindblad. Contrario a la suposición natural de esperar que el signo del torque neto dependa del perfil de densidad superficial del gas, cálculos detallados para discos de diversos perfiles de densidad superficial de genuino interés muestran que el torque neto de las resonancias de Lindblad, en el marco discutido, es negativo, es decir, la migración es hacia adentro (Ward, 1997). Con respecto a la contribución de la resonancia de corrotación al torque neto, para un disco bidimensional ésta es nula para discos con una densidad superficial con caída kepleriana $\left(\Sigma \propto r^{-3 / 2}\right)$ (Goldreich y Tremaine, 1979). Por supuesto, este resultado no es válido para discos tridimensionales, pero en tal caso la contribución es significativamente menor, aunque no despreciable, que la correspondiente a las resonancias de Lindblad y, por sobre todo, no revierte el sentido de la migración (Ward, 1997, Tanaka y otros, 2002). La migración orbital de un planeta sumergido en un disco de gas por acción del torque neto calculado según el marco discutido se conoce como migración de Tipo I. La velocidad o tasa de migración en este régimen es proporcional a la masa del planeta y por lo tanto su escala de tiempo, o tiempo de deriva, resulta inversamente proporcional a la masa del planeta; esto es, la migración de Tipo I es más rápida cuanto mayor sea la masa del planeta. Ahora bien, en virtud de la hipótesis fundamental de que la interacción con el disco es lo suficientemente débil como para considerar un tratamiento lineal de las ecuaciones hidrodinámicas y suponer que la distribución de gas en el disco es sólo levemente alterada, es claro que una migración de Tipo I sólo podrá ocurrir para planetas de masa suficientemente baja (típicamente del orden de la masa terrestre $M_{\oplus}$ ), aunque el valor exacto dependerá de las propiedades del disco) donde el intercambio de momento angular gravitatorio entre el planeta y el disco es despreciable con respecto a la redistribución de momento angular debido a la viscosidad del gas. En esta situación la densidad superficial del gas permanece casi sin perturbar y por lo tanto el gas está presente en todas las localizaciones resonantes satisfaciéndose así las condiciones para el cómputo del torque neto de acuerdo al régimen de migración de Tipo I. En la situación opuesta, para planetas de masa suficientemente grande (típicamente por encima de una fracción de la masa de Júpiter) donde el torque gravitacional predomina localmente sobre la redistribución de momento angular por viscosidad, el hecho de que, como hemos mencionado previamente, la acción gravitacional del planeta tiende a repeler el gas en su vecindad, provoca que la densidad superficial del gas decaiga en la vecindad de $r=r_{p}$, formando una brecha, esto es, una región anular en la cual la densidad superficial del gas es mucho menor que su valor no perturbado original. En tal situación, en las localizaciones resonantes más próximas al planeta habrá una deficiencia de material gaseoso; éstas contribuirán poco o nada al torque total y por lo tanto sólo las resonancias de Lindblad de órdenes $m$ más bajo contribuirán significativamente. Así, el torque neto será sensiblemente menor al predicho por el modelo de la migración Tipo I. Claramente, una vez que comienza a abrirse una brecha en la distribución del gas, la hipótesis que sustenta el tratamiento lineal de la interacción planeta-disco resulta inválida y el torque debe ser calculado autoconsistentemente junto con un modelo de la evolución del disco de gas, el cual involucra a las ecuaciones no lineales de la hidrodinámica. El régimen de migración establecido cuando la brecha se encuentra abierta se conoce como migración de Tipo 
II. En este caso la tasa de migración y su tiempo de deriva resultan independientes de la masa del planeta; para un disco dado, sólo es función de su viscosidad. El modelo unificado de Ward (1997) muestra que, conforme un protoplaneta aumenta su masa acretando material del disco, evoluciona de un régimen de Tipo I a un régimen de Tipo II.

Para finalizar el marco teórico indiquemos que Goldreich y Tremaine (1979) establecieron que para discos gaseosos bidimensionales fríos, condición expresada por la desigualdad

$$
\frac{c_{s}}{\Omega r} \ll 1
$$

donde $c_{s}$ es la velocidad del sonido del medio y $\Omega$ la frecuencia orbital a la distancia $r$, el torque descrito por la teoría lineal de ondas de densidad es equivalente a la formulación resonante en discos de partículas, aunque en este caso no se establecen ondas de densidad excitadas que transportan el momento angular al disco, sino que la transferencia ocurre directamente sobre los planetesimales localizados en las resonancias. Por otra parte, en un disco de planetesimales, son las colisiones mutuas quienes juegan un papel análogo a la viscosidad en el disco para disipar energía e impedir la apertura de una brecha.

El marco teórico anterior clarifica el objetivo propuesto en el trabajo de Cionco y Brunini (2002). A continuación describiremos la metodología y los resultados encontrados. Como ya hemos indicado, el modelo propuesto por los investigadores consta de un planeta de masa $M$ orbitando en torno a una estrella de tipo solar en una órbita inicial cuasi-circular a $5 \mathrm{UA}$. El planeta se encuentra asimismo sumergido en un disco de planetesimales de densidad superficial constante $\Sigma=12 \mathrm{~g} \mathrm{~cm}^{-2}$, valor consistente con un modelo de masa nebular mínima $\left(\sim 0.01 M_{\odot}\right)$, que se extiende entre 2 y $11 \mathrm{UA}$. Esta elección de los límites garantiza la inclusión de todas las resonancias planeta-disco relevantes para el cálculo del torque teórico. Las simulaciones se llevaron a cabo para tres tipos de cuerpos planetarios: para un cuerpo de masa equivalente a la masa de Júpiter, $M_{\mathrm{J}}$, un cuerpo de masa igual a la masa de Saturno, $M_{\mathrm{S}}$, y un cuerpo de masa correspondiente a diez masas terrestres, $M_{\mathrm{c}}=10 M_{\oplus}$. Por su parte el disco de planetesimales es construido a partir de cierto número $N$ de planetesimales de masas iguales $m$ de manera tal que la granularidad $M / m$ de la simulación sea mayor que $10^{2}$, puesto que esto permite considerar el disco no autogravitante (Ida y Makino, 1993). Las simulaciones se llevaron a cabo sobre un período de $10^{5}$ años, con un código de segundo orden diseñado por Brunini, al cual se le realizaron modificaciones para el correcto tratamiento de los encuentros. Específicamente, cuando un planetesimal cae dentro de una esfera del orden del radio de Hill del planeta, el encuentro se calcula como una interacción de dos cuerpos más una perturbación debida a la estrella central. El código permite también acreción planetaria y eyección de cuerpos en órbitas hiperbólicas. El resultado más relevante de las simulaciones es la tasa de variación del semieje mayor del planeta, $\dot{a}$, el cual determina un tiempo característico de deriva $\tau$ dado por

$$
\tau=\frac{a}{|\dot{a}|},
$$

esto es, el tiempo necesario para que el cambio en el semieje sea del mismo orden que su valor; y el torque $\Gamma$ ejercido por el disco sobre el planeta en una órbita cuasi-circular 
de frecuencia orbital $\Omega$, dado por

$$
\Gamma=\frac{1}{2} a \Omega M \dot{a}
$$

En efecto, la estructura aplanada del disco permite suponer que la totalidad del torque ejercido por el disco sobre el planeta, moviéndose muy cerca del plano $x-y$, es debido a su componente $z$. De este modo es lícito suponer que, en virtud del torque $\Gamma$, el planeta experimenta, sobre un órbita aproximadamente circular, una aceleración tangencial $T$ en la dirección tangente al movimiento $\hat{u}_{\mathrm{T}}$ que es transversal al radio vector $\mathrm{y}$, por tanto, está relacionada con dicho torque por la ecuación

$$
\Gamma=a T M
$$

Debido a $T$ el planeta experimenta un cambio en su velocidad $\mathrm{d} \mathbf{v}=T \mathrm{~d} t \hat{u}_{\mathrm{T}}$. Se sigue entonces de la ecuación de energía del problema de los dos cuerpos

$$
\mathbf{v}^{2}=\mu\left(\frac{2}{r}-\frac{1}{a}\right), \quad \text { siendo } \mu=\Omega^{2} a^{3},
$$

que para cada uno de una sucesión de impulsos instantáneos

$$
2 \mathbf{v} \cdot \mathrm{d} \mathbf{v}=\mu \frac{1}{a^{2}} \mathrm{~d} a .
$$

Como, en nuestra situación, $\mathbf{v} \cdot \mathrm{d} \mathbf{v}=\left(v \hat{u}_{\mathrm{T}}\right) \cdot\left(T \mathrm{~d} t \hat{u}_{\mathrm{T}}\right)=v T \mathrm{~d} t$, se sigue de la ecuación anterior que

$$
T=\frac{1}{2} \frac{\mu}{v a^{2}} \dot{a}=\frac{1}{2} \frac{\Omega^{2}}{v} a \dot{a} .
$$

Finalmente, considerando la órbita del planeta esencialmente circular, $v=\Omega a$, con lo cual obtenemos

$$
T=\frac{1}{2} \Omega \dot{a} .
$$

Reemplazando esta ecuación en (3-7) obtenemos la ecuación (3-6).

La tasa de migración se obtiene por un ajuste de mínimos cuadrados sobre el semieje dentro del intervalo en el cual la misma exhiba una variación constante. En este sentido los autores observan respecto a la variación del semieje en sus simulaciones y pruebas, que existen dos estadios principales: una fase transitoria donde están presentes oscilaciones y saltos del semieje producidos por el dominio de los efectos estocásticos relacionados principalmente con la masa y distribución inicial de las partículas que simulan al disco, y una fase estacionaria donde se presenta una variación casi constante del semieje con saltos y oscilaciones de mucha menor amplitud, donde efectivamente determinan la tasa de migración planetaria por la fricción dinámica con el disco. Los autores mencionan que para las simulaciones de masas $M_{\mathrm{J}}$ y $M_{\mathrm{S}}$ requieren de al menos $N=10000$ planetesimales para modelar la situación correctamente, mientras que para la masa $M_{\mathrm{c}}$ se requiere un valor $N=20000$. Sin embargo, los saltos en el semieje nunca desaparecen completamente. Sobre este punto volveremos al considerar las simulaciones con nuestro propio código. En líneas generales, las simulaciones realizadas por los autores, muestran que los planetas migran hacia el interior del disco desarrollando un patrón espiral en el sentido contrario a la traslación planetaria. El disco se ve muy 


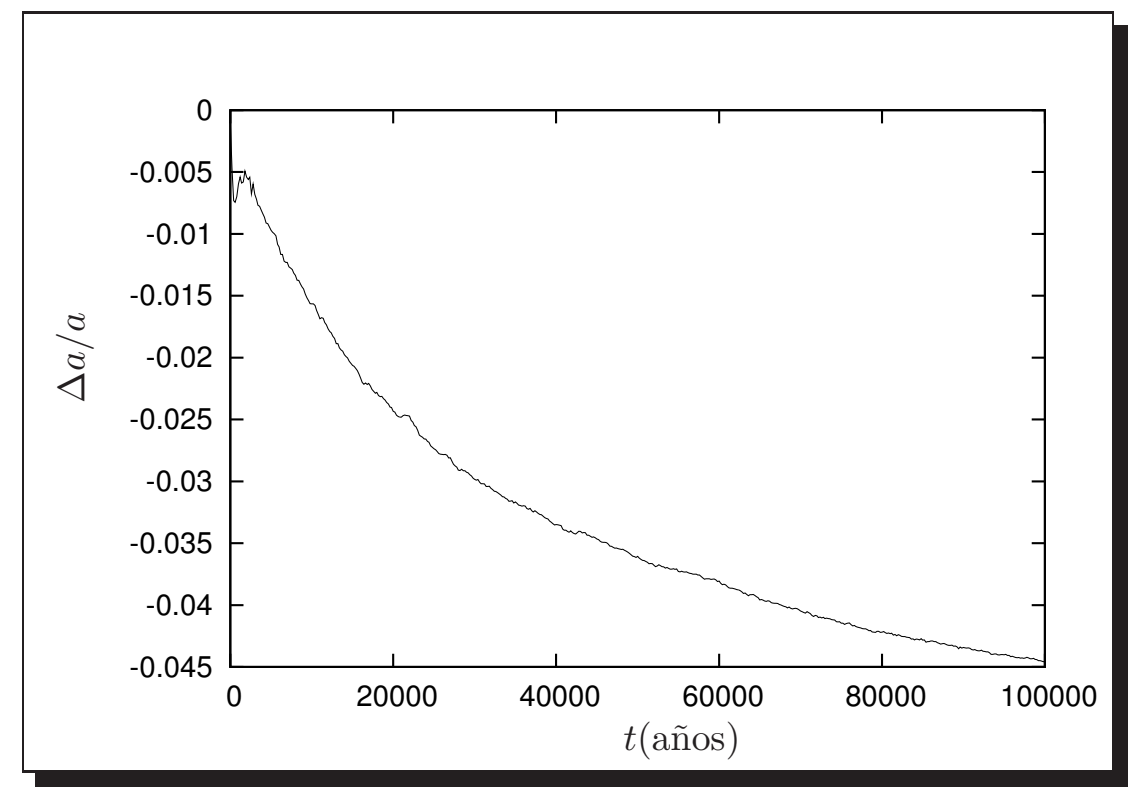

Figura 3.1. Variación relativa del semieje mayor respecto a su valor inicial en la migración del cuerpo planetario $M_{\mathrm{J}}$.

perturbado en las cercanías de las resonancias de Lindblad y la de corrotación y una brecha en la densidad superficial del disco aparece en la zona de superposición de resonancias, siendo la misma muy profunda para los casos de las masas $M_{\mathrm{J}}$ y $M_{\mathrm{S}}$ y menos pronunciada para el caso de la masa $M_{\mathrm{c}}$. Las velocidades de migración determinadas a partir del promedio de al menos cuatro corridas en cada caso, muestra que la escala de tiempo de migración se encuentra en el orden de $\sim 10^{6}$ años. Aunque los planetas predan el disco, la gran mayoría de los planetesimales que cruzan la órbita del planeta son dispersados y no acretados. La comparación del torque resonante obtenido de la simulación con respecto al valor computado por el modelo teórico lineal muestra una reducción del torque medido por un factor de 0.38 para $M_{\mathrm{c}}, 0.04$ para $M_{\mathrm{S}}$ y 0.01 para $M_{\mathrm{J}}$. Estos resultados indican que mientras que para $M_{\mathrm{c}}$ la disminución del torque es lo esperado para migradores de Tipo I en discos gaseosos, los cuerpos más masivos se comportan como migradores de tipo II en discos gaseosos.

Con el fin de poner a prueba nuestro código, en este escenario descrito, consideramos el caso del perturbador planetario de masa igual a la masa de Júpiter, $M_{\mathrm{J}}$, ubicado inicialmente a 5 UA y sumergido en un disco de densidad constante $\Sigma=12 \mathrm{~g} \mathrm{~cm}^{-2}$ extendiéndose entre 2 y 11 UA. El disco es construido a partir de la distribución de $N=20000$ partículas siguiendo un perfil de densidad constante, con lo cual la masa de cada una de ellas es de $m=0.008 M_{\oplus}$ y por lo tanto la granularidad del sistema es $M / m \sim 38000{ }^{4}$. Las excentricidades e inclinaciones orbitales iniciales de las partículas son generadas al azar siguiendo un perfil gaussiano con dispersiones de valores 0.01 y 0.005 respectivamente, en tanto los restantes elementos orbitales son generados al azar siguiendo una distribución de probabilidad uniforme en el rango correspondiente. La simulación es llevada a cabo a lo largo de 100000 años con un paso temporal $\Delta t=0.1$ años. La Fig. 3.1 muestra la variación relativa del semieje mayor

\footnotetext{
${ }^{4}$ Recordemos que $M_{\mathrm{J}}=313 M_{\oplus}$.
} 


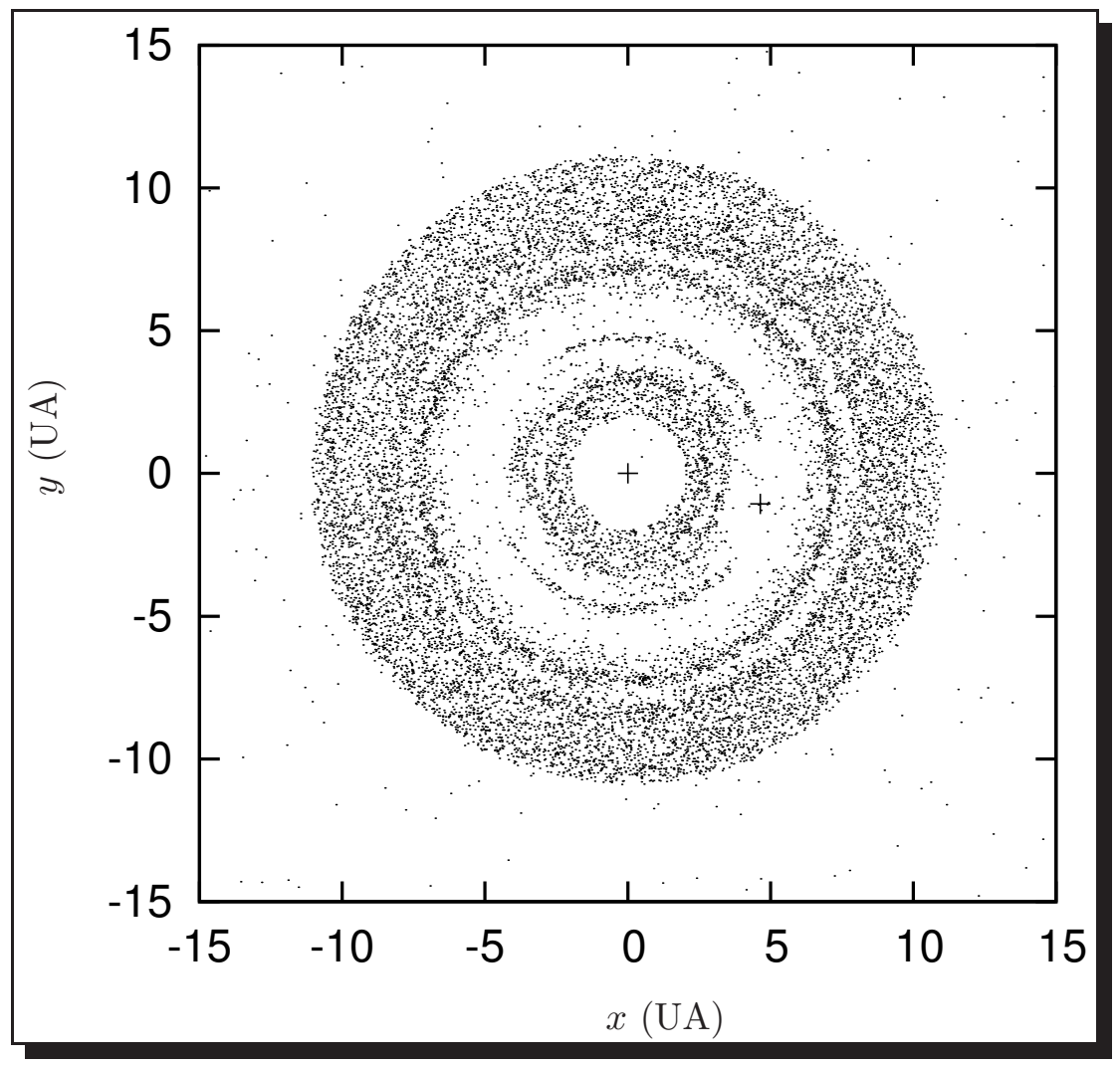

Figura 3.2. Estado el disco de planetesimales en el plano $x-y$ resultante de la migración del cuerpo planetario $M_{\mathrm{J}}$ al finalizar la simulación en $10^{5}$ años. Los signos '+' indican la localización de la estrella central y el cuerpo planetario. Nótese la presencia de planetesimales corrotadores agrupados en las cercanías de los puntos lagrangianos $L_{4}$ y $L_{5}$ del planeta.

del perturbador planetario a lo largo del tiempo. La velocidad de migración, obtenida siguiendo el procedimiento descrito anteriormente en $[20-80] \times 10^{3}$ años, arroja el valor

$$
\dot{a}=-1.22 \times 10^{-6} \mathrm{UA} \mathrm{año}^{-1},
$$

lo cual da un tiempo característico de deriva de

$$
\tau=4.1 \times 10^{6} \text { años, }
$$

resultados compatibles con los obtenidos por Cionco y Brunini (2002). Es interesante destacar que el resultado anterior es obtenido a partir de una única simulación y no del promedio de un conjunto de ellas, como es realizada por los autores mencionados, ya que en nuestra simulación la variación del semieje, luego de la fase inicial, tiene un comportamiento suave. Esto se debe en última instancia a la introducción en nuestro código de la función encuentro para el tratamiento de los encuentros, tal como hemos descrito en la sección 2.3 del capítulo 2 . Como hemos indicado, tal función mantiene la naturaleza simpléctica del integrador a la vez que permite seguir correctamente un encuentro. Por el contrario el esquema más simple que calcula dicho encuentro como una interacción de dos cuerpos más una perturbación debida a la estrella central, implementado en el código original de Cionco y Brunini, termina destruyendo la simplecticidad 


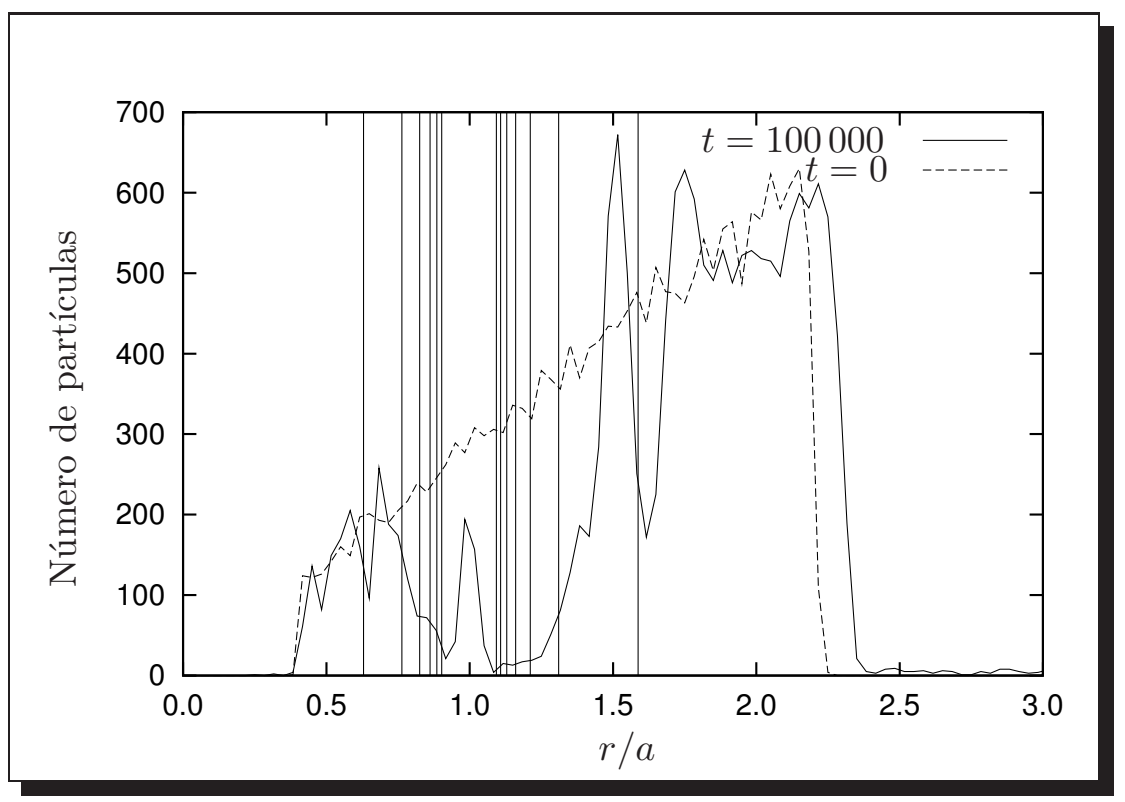

Figura 3.3. Distribución del número de partículas a lo largo del disco, normalizadas al valor del semieje mayor de la órbita del planeta migrante, al tiempo inicial y final de la simulación para el cuerpo planetario $M_{\mathrm{J}}$. La localización de las resonancias de Lindblad están indicadas por las líneas verticales.

del código y es origen de los saltos, luego de la fase transitoria, en la evolución del semieje reportados por los autores.

Continuando con el análisis de los resultados de la simulación con nuestro código, la figura 3.2 muestra la depredación del disco de partículas debido al patrón espiral que tiende a desarrollar el planeta en su migración, en tanto que la figura 3.3 muestra la distribución del número de partículas del disco con la distancia radial al tiempo inicial y final de la simulación, junto con la localización de las resonancias de Lindblad en el disco. Como puede apreciarse, una profunda brecha surge en la zona de superposición de las resonancias. Finalmente, en la figura 3.4 se muestra el estado dinámico del disco en el plano $a-e$ al finalizar la simulación. Tal gráfico da cuenta de la excitación de los planetesimales dentro de la zona de cruce con el perturbador planetario, cuyo límite es indicado por la línea continua ${ }^{5}$. Dentro de esta zona los planetesimales se ven fuertemente dispersados, aumentando a la vez su excentricidad y semieje. Por otra parte, también se observa excitación de la excentricidad en zonas del semieje resonantes al planeta por fuera de la zona de cruce.

\subsection{Migración planetaria en un disco masivo.}

En esta sección mostraremos la capacidad de nuestro código en un segundo escenario de migración más complejo que involucra a los planetas gigantes del sistema solar según el artículo de Gomes y otros (2004).

\footnotetext{
${ }^{5}$ Ver apéndice E para la definición y cálculo de la zona de cruce planetario.
} 


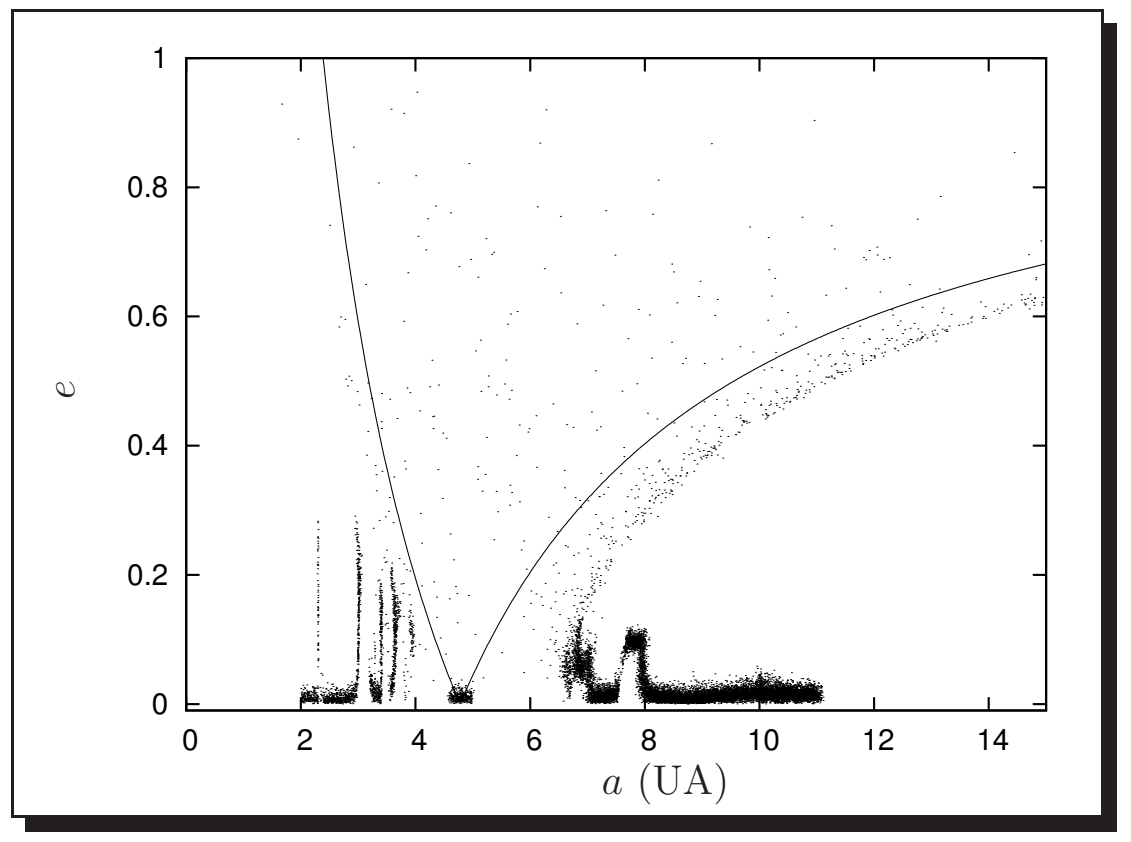

Figura 3.4. Estado dinámico en el plano $(a, e)$, de semiejes $a$ y excentricidades $e$, del disco de planetesimales al final de la simulación $\left(t=10^{5}\right.$ años) correspondiente al cuerpo planetario $M_{\mathrm{J}}$. La línea continua indica el borde de la zona de cruce con el planeta.

Antes de describir las simulaciones consideradas procederemos a dar el contexto teórico y observacional que involucran a las mismas. En los últimos años, el número de objetos conocidos más allá de la órbita de Neptuno, denominados colectivamente como objetos transneptunianos (TNOs, del inglés trans-Neptunian objects), ha crecido enormemente en virtud de los avances de las técnicas de observación astronómica. Aunque su existencia teórica fue sugerida en los años 50 del siglo pasado por Edgeworth y Kuiper (ver Davies y otros (2008) para una revisión histórica de los primeras ideas concernientes a esta región), sólo en tiempos más recientes se descubrió el primer objeto transneptuniano (descontando a Plutón y su satélite Caronte): 1992 QB 1 (Jewitt y Luu, 1993). Desde entonces el número de objetos conocidos sobrepasa el millar, tal como lo muestra la lista de objetos transneptunianos actualizada constantemente en el Minor Planet Center $^{6}$. Con tamaños en el rango de las decenas a unos miles de kilómetros todos ellos comparten una característica que los define, a saber, los perihelios de sus órbitas son mayores que el semieje mayor de Neptuno ( $a=30 \mathrm{UA})$ y sus órbitas se extienden hasta unas centenas de unidades astronómicas. Actualmente, la dinámica de sus órbitas permite diferenciar estos objetos en, al menos, cuatro clases (Delsanti y Jewitt, 2006), lo cual se ilustra en la Figura 3.5.

${ }^{6}$ El Minor Planet Center (MPC) es un organismo del Smithsonian Astrophysical Observatory (SAO), que forma parte del Center for Astrophysics (CfA) junto con el Harvard College Observatory (HCO). Bajo el auspicio de la International Astronomical Union (IAU), el MPC es la institución encargada de recopilar observaciones de los cuerpos menores del Sistema Solar, calcular sus órbitas y publicar los resultados. En su sitio Web, http://www.minorplanetcenter.net/iau/mpc.html, ofrece numerosos servicios, uno de los cuales es el catálogo en línea de objetos transneptunianos, accesible en http://www.minorplanetcenter.net/iau/lists/MPLists.html. 
I. En primer lugar tenemos los objetos resonantes, los cuales se encuentran en resonancias de movimiento medio con Neptuno. De entre ellas, la resonancia 2:3, con localización nominal en 39.4 UA, es la más densamente poblada (del orden del centenar de objetos). Sus miembros son conocidos como plutinos puesto que Plutón pertenece a tal población.

II. En segundo lugar, podemos distinguir a los objetos del cinturón de Edgeworth-Kuiper clásico, los cuales son objetos no resonantes con bajas excentricidades $(e \lesssim 0.2)$ que se encuentran en la región comprendida entre la resonancia de movimiento medio 3:2 a 39.4 UA y la resonancia 1:2 a 47.6 UA (sin incluir a las mismas). Las observaciones muestran que en esta región conviven dos poblaciones, una de bajas inclinaciones, $i<4^{\circ}$, conocida como población "fría" ( cold), y otra de altas inclinaciones, tanto como $30^{\circ}$ o más, denominada población "caliente" (hot). El conjunto de objetos de las poblaciones resonantes y del cinturón de Edgeworth-Kuiper clásico se conocen, colectivamente, como objetos del cinturón de Edgeworth-Kuiper (KBOs, del inglés Kuiper belt objects).

III. La tercera clase la constituyen los objetos del disco dispersado (SDOs, del inglés scattered disk objects), los cuales tienen perihelios en el rango de 30 a $40 \mathrm{UA}$ con excentricidades e inclinaciones altas.

IV. Finalmente, una cuarta clase corresponde a los llamados objetos del disco dispersado extendido (E-SDO, del inglés extended scattered disc objects) o, con más propiedad, objetos distantes separados (DDO, del inglés distant detached objects) los cuales poseen distancias perihélicas de más de 40 UA. Con perihelios tan lejanos de la órbita de Neptuno, su influencia gravitacional no es significativa y de aquí que se consideren "separados" respecto de las otras poblaciones.

La determinación de los mecanismos que originaron la estructura actual de los objetos transneptunianos constituye un tema activo y de ningún modo está completa. Esto se debe a que un buen modelo debe poder explicar ciertas particularidades de la dinámica actual de la región reseñadas por Levison y otros (2008):

I. La presencia de objetos con altas excentricidades en las resonancias externas de movimientos medios con Neptuno (2:3, 3:5, 4:7, 1:2, 2:5, etc.). Un análisis de su dinámica muestra que estas resonancias son estables, pero no explica por qué ni cómo existen objetos en estas resonancias sobre órbitas con excentricidades tan grandes como lo permiten los criterios de estabilidad.

II. La excitación de las excentricidades en el cinturón de Kuiper clásico. La excentricidad media de la población del cinturón clásico es $\sim 0.07$. Aun cuando la excentricidad media actual de los objetos de esta población es relativamente pequeña, la misma es, al menos, un orden de magnitud mayor que aquella que debió haber existido en la formación de estos objetos. La dinámica actual es estable en la región del cinturón clásico, de modo que sin mecanismos perturbativos adicionales, las pequeñas excentricidades primordiales deberían haber sido preservadas hasta los tiempos actuales.

III. La carencia de objetos del cinturón de Kuiper clásico sobre órbitas cuasi-circulares $(e \lesssim 0.05)$ en el rango de semiejes $44 \lesssim a \lesssim 48$. En efecto, más allá de 44 UA la excentricidad de la población de objetos tiende a incrementarse con el semieje. Este 


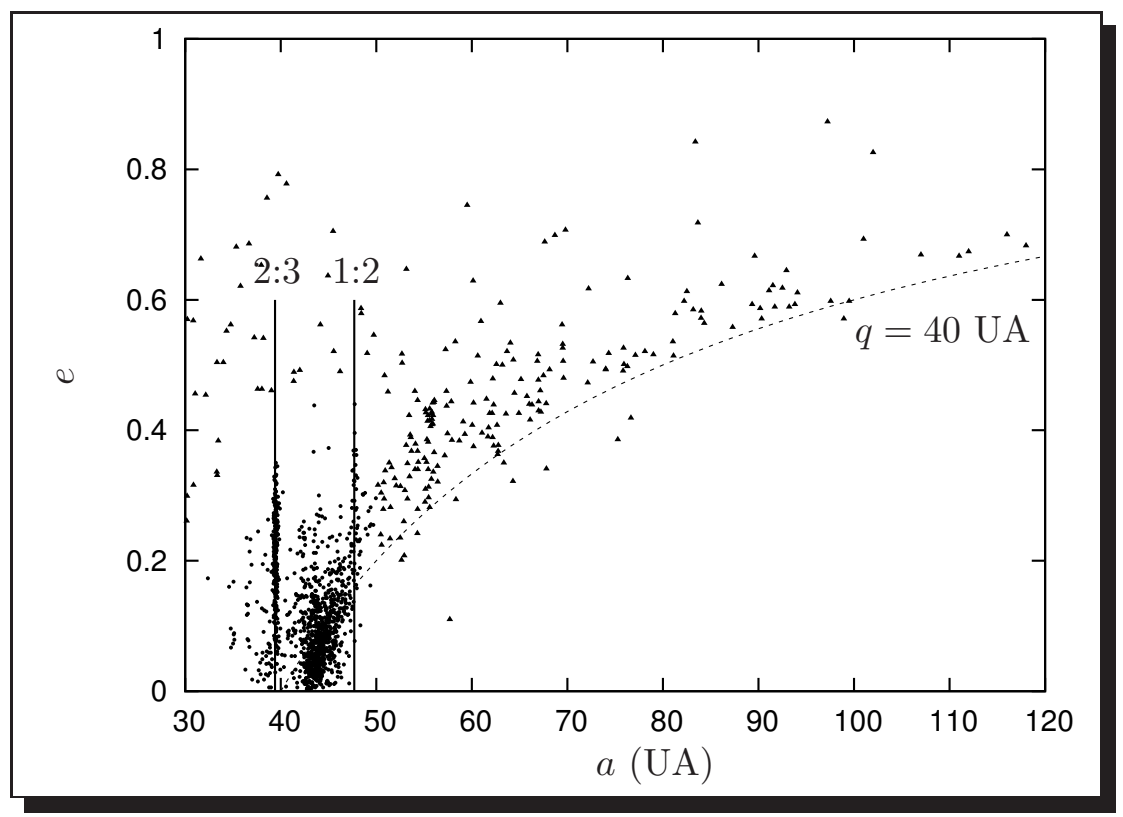

Figura 3.5. Objetos transneptunianos en el plano $(a, e)$ de semiejes $a$ y excentricidades $e$, de acuerdo a las listas del MPC a finales de enero de 2014. Los círculos representan la población resonante y los objetos del cinturón de Kuiper clásico. Las líneas verticales muestran las localizaciones nominales de las resonancias 2:3 y 1:2 con Neptuno. Los plutinos constituyen la población de la resonancia 2:3, mientras que la resonancia 1:2 establece un borde externo a la distribución de objetos del cinturón clásico. Los triángulos representan la población del disco dispersado y del disco dispersado extendido. La curva a trazos corresponde a valores de $a$ y $e$ con un perihelio $q$ de $40 \mathrm{UA}$.

hecho no puede ser explicado ni como consecuencia de un sesgo observacional ni por consideraciones de estabilidad dinámica? .

IV. El límite exterior del cinturón de Kuiper clásico coincidiendo con la localización de la resonancia externa de movimientos medios 1:2 de Neptuno. Sólo objetos con grandes excentricidades asociados a la población del disco dispersado u objetos distantes separados parecen existir más allá de dicho límite. La carencia de objetos con bajas excentricidades más allá de la resonancia 1:2 no puede ser explicada como consecuencia de un sesgo observacional y un análisis de estabilidad muestra que tal región resulta ser estable, incluso a moderadas excentricidades. Por lo tanto, cualquier población primordial distante debería haber permanecido allí hasta la actualidad.

v. La distribución de inclinaciones en el cinturón de Kuiper clásico y la correlación de propiedades físicas. Como ya hemos indicado, las observaciones sugieren la coexistencia de dos poblaciones en el cinturón de Kuiper clásico: una población dinámicamente fría (cold), constituida por objetos en órbitas con inclinaciones $i<40^{\circ}$, y una población

\footnotetext{
${ }^{7} \mathrm{Si}$ esta característica fuese simplemente la consecuencia de un sesgo observacional que favorece el descubrimiento de objetos con órbitas de distancias perihélicas $q$ más pequeñas, el límite inferior de la distribución de tales objetos en el plano $(a, e)$ seguiría una curva dada por un valor de $q$ constante. Sin embargo, las observaciones indican que la excentricidad en la región considerada crece más fuertemente con el semieje mayor que respecto al valor que tomaría si siguiese una curva de $q$ constante.
} 
dinámicamente caliente (cold) de mayores inclinaciones. Además la evidencia observacional muestra que estas dos poblaciones tienen diferente distribución de tamaños y diferentes colores. La población hot consiste en objetos más grandes que la población cold y, mientras que la población hot muestra un amplio rango de colores, de rojo a gris, los objetos de la población cold son principalmente rojos. Estas diferencias en las propiedades físicas sugiere que las dos poblaciones tienen orígenes distintos.

VI. El llamado problema del déficit de masa de la región transneptuniana. Las estimaciones de la masa total contenida actualmente en el cinturón de Kuiper indican valores en el rango de $0.01 M_{\oplus}$ a $0.1 M_{\oplus}$. Aun dentro de la incerteza de este valor, los modelos indican una diferencia de 2 a 3 órdenes de magnitud con respecto a la masa primigenia necesaria para la formación in situ del cinturón de Kuiper. En particular, para que los objetos hoy presentes se hayan formado en un tiempo de $10^{7}-10^{8}$ años, el cinturón de Kuiper tuvo que contener originalmente de 10 a $30 M_{\oplus}$ de material sólido en un disco dinámicamente frío. Puesto que la mayor parte del cinturón de Kuiper actual es dinámicamente estable, no es posible explicar la remoción de más del $99 \%$ de su masa primordial en este contexto.

Aun sin disponer de un modelo completo que de cuenta de todas estas características, es un consenso general que la migración planetaria de Neptuno desde su posición de nacimiento original hasta la actual fue la responsable de "esculpir" la región transneptuniana, generando las características observacionales actuales (Armitage, 2010).

En Malhotra $(1993,1995)$ se plantea por primera vez que las excentricidades de los objetos resonantes del cinturón de Edgeworth-Kuiper pueden ser explicadas como consecuencia de una migración de Neptuno hasta su posición actual de al menos 5 UA.

En efecto, bajo ciertas condiciones un cuerpo capturado en una resonancia externa de movimiento medio de un planeta, continúa atrapado en dicha resonancia conforme el planeta migra hacia afuera y, al mismo tiempo, la excentricidad de dicho cuerpo se incrementa (Murray y Dermott, 1999). Específicamente, para una partícula en la resonancia externa $j:(j+1)$, la excentricidad evoluciona según la ecuación (Malhotra, 1995)

$$
e^{2}=e_{0}^{2}+\frac{1}{j+1} \log \frac{a_{N}}{a_{N, 0}},
$$

donde $e_{0}$ es la excentricidad de la partícula al instante en que la misma es capturada en la resonancia, siendo $a_{N, 0}$ el semieje mayor de Neptuno cuando dicho evento ocurre, e y $a_{N}$ la excentricidad de la partícula y el semieje mayor de Neptuno al mismo instante, posterior a la captura. Aplicando esta ecuación a Plutón, asumiendo la captura en resonancia 2:3 $(j=2)$ en una órbita circular en cierta posición de Neptuno a determinar y la consiguiente evolución hasta alcanzar el valor actual de la excentricidad $e=0.25$ con Neptuno en la posición actual $a_{N}=30 \mathrm{UA}$, encontramos que Neptuno se encontraba a $a_{N, 0} \approx 25 \mathrm{UA}$ al momento de la captura. Por lo tanto, Neptuno ha migrado al menos 5 UA.

La ecuación (3-11), de la cual deriva el resultado anterior, requiere de ciertas aproximaciones en el marco del modelo conocido como aproximación adiabática de la captura en resonancia, la cual implica, en el contexto descrito, un único planeta sobre una órbita circular migrando lenta y monótonamente. Así, el modelo no puede ser aplicado si la excentricidad del planeta o la inclinación de la partícula es grande. Pero, además, si conforme el planeta migra, éste se encuentra con otros planetas u otros planetesimales 
de mayores dimensiones que el resto presente en el disco, entonces el semieje mayor del mismo sufre saltos al igual que las localizaciones de las posiciones resonantes; si la amplitud de tales saltos son mayores que el ancho de la resonancia, entonces las partículas atrapadas en las mismas serán liberadas (Murray-Clay y Chiang, 2005). Por otra parte, aunque el escenario de barrido de las resonancias puede explicar la existencia de la población resonante, no puede dar cuenta de la distribución orbital de los objetos no resonantes del cinturón de Kuiper clásico. Claramente, el estudio de un escenario más completo requiere invariablemente de simulaciones numéricas que permitan, contrastando con las características observables del cinturón de Kuiper, determinar que tipo de migración ocurrió en el sistema solar y, así, en última instancia derivar la masa y estructura del disco protoplanetario primigenio.

Consideremos, entonces, el trabajo de Gomes y otros (2004), en donde los autores analizan, a través de un conjunto de simulaciones, distintos regímenes de migración que Neptuno puede seguir, dependiendo de la masa presente en el disco protoplanetario remanente del proceso de formación planetaria. Sus resultados les permiten sugerir que la actual posición de Neptuno y el problema del déficit de masa de la región transneptuniana pueden ser explicados suponiendo que el disco protoplanetario tenía su borde exterior alrededor de las $30 \mathrm{UA}$. Nótese que este truncamiento en la distribución de partículas del disco no contradice la existencia del cinturón de Kuiper actual, a más de 40 UA, puesto que en el contexto descrito, todo el cinturón de Kuiper habría sido generado a partir de la dispersión de los cuerpos presentes en las cercanías de dicho borde durante el pasaje de Neptuno en su migración hacia el exterior. En particular, nos interesa destacar, entre el conjunto de simulaciones presentadas, aquellas que consideran una configuración inicial más compacta de los planetas gigantes en un disco de planetesimales que se extiende entre 20 y 50 UA, esto es, hasta el borde actual del cinturón de Kuiper clásico, con un perfil de densidad superficial que varía con la distancia radial $r$ sobre el plano del disco como $r^{-1}$. Para dicho sistema se efectúan distintas simulaciones donde se varía la masa total del disco, desde $40 M_{\oplus}$ hasta $200 M_{\oplus}$. Los resultados muestran que la evolución temporal del semieje mayor de Neptuno difiere según la masa total del disco considerado como sigue:

I. Para un rango entre $40 M_{\oplus}$ y $45 M_{\oplus}$, después de una rápida migración, Neptuno desacelera su tasa de migración hacia el exterior hasta alcanzar un valor casi nulo, alcanzando así un cuasi-asintótico semieje mayor que lo posiciona dentro del disco pero alejado del borde exterior del mismo. La región del disco por fuera de la órbita de Neptuno preserva su perfil de densidad original, mientras que en la región interior está casi completamente despoblada. Tal régimen de migración es denominado por los autores como migración amortiguada.

II. En los discos con masa mayor o del orden de $50 M_{\oplus}$, por el contrario, la migración continúa y Neptuno alcanza una posición final un poco más allá del borde exterior del disco. Este régimen es denominado por los autores como migración forzada.

III. Para discos con masas mayores que unas $100 M_{\oplus}$ el semieje mayor de Neptuno ya no se incrementa monótonamente con el tiempo, sino que Neptuno alcanza rápidamente el borde exterior del disco y revierte su dirección migrando hacia el interior, para luego volver a migrar hacia el exterior hasta alcanzar nuevamente el límite exterior del disco. Este régimen de migración es denominado, por los autores, migración en fuga. Aquí el 


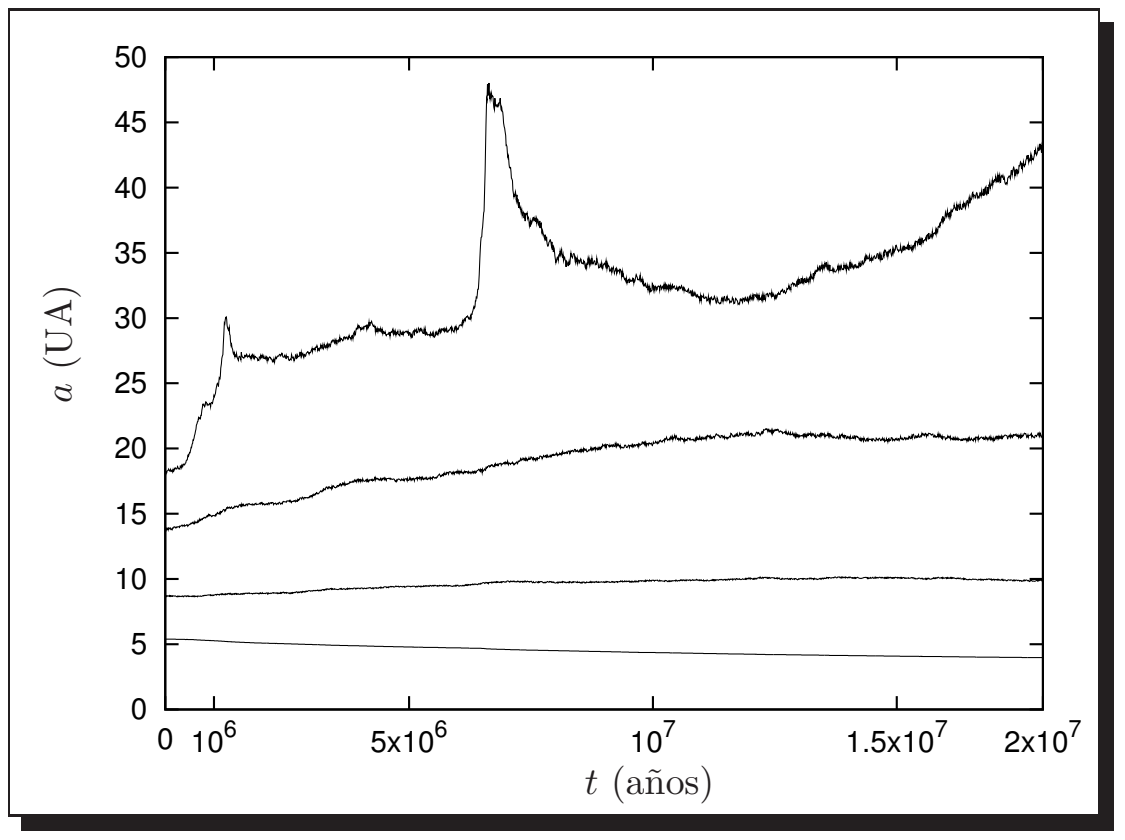

Figura 3.6. Evolución de los semiejes mayores de los cuatro planetas gigantes en un disco de planetesimales de $200 \mathrm{M}_{\oplus}$ extendido inicialmente entre 20 y 50 UA. Las curvas se corresponde, de abajo hacia arriba, a Júpiter, Saturno, Urano y Neptuno.

proceso de migración es autosustentado, es decir, una vez iniciada la migración, ésta procede sin la necesidad de que otros planetas estén presentes.

Con el fin de testear nuestro código en el escenario planteado consideramos, en particular, una simulación de la migración en fuga de Neptuno para un disco de $200 M_{\oplus}$ en la región de 20 a 50 UA, tal como es descrita en el trabajo referenciado. A saber, se consideran órbitas iniciales de excentricidad e inclinación nulas para Júpiter, Saturno, Urano y Neptuno con semiejes mayores de 5.4, 8.7, 13.8 y 18.1 UA, respectivamente. El disco de planetesimales se extiende desde 12.5 a 50 UA. Puesto que, debido a la acreción planetaria, el disco debería estar despoblado en la región que contiene a los planetas, se supone que la región interna de 12.5 a 20 UA contiene unas $5 M_{\oplus}$ con un perfil de densidad superficial que decae con la distancia radial sobre el plano del disco como $r^{-1.5}$. Por otra parte, en la región externa, de 20 a 50 UA, se supone que el disco es tan masivo como para contener $200 M_{\oplus}$ con un perfil de densidad superficial proporcional a $r^{-1}$. Entonces, la región exterior del disco es simulada utilizando $N=10000$ partículas de igual masa, la cual impone entonces que el disco interior sea simulado con $N=250$ partículas de idéntica masa. La excentricidades e inclinaciones orbitales iniciales de las mismas siguen un perfil gaussiano con dispersiones de valores 0.01 y 0.05 respectivamente, con los restantes elementos orbitales asignados según una distribución de probabilidad uniforme en el rango correspondiente. La simulación procede sobre $20 \times 10^{6}$ años con un paso temporal $\Delta t=0.5$ años. Como en las simulaciones precedentes, las partículas interactúan con los planetas pero no entre ellas. La Figura 3.6 ilustra la evolución temporal de los cuatro planetas gigantes. Como era de esperarse, Saturno, Urano y Neptuno tienden a migrar hacia el exterior, mientras que Júpiter lo hace hacia el interior del disco. Pero más importante aún, nuestra simulación reproduce el régimen de migración en fuga de Neptuno: aproximadamente a 


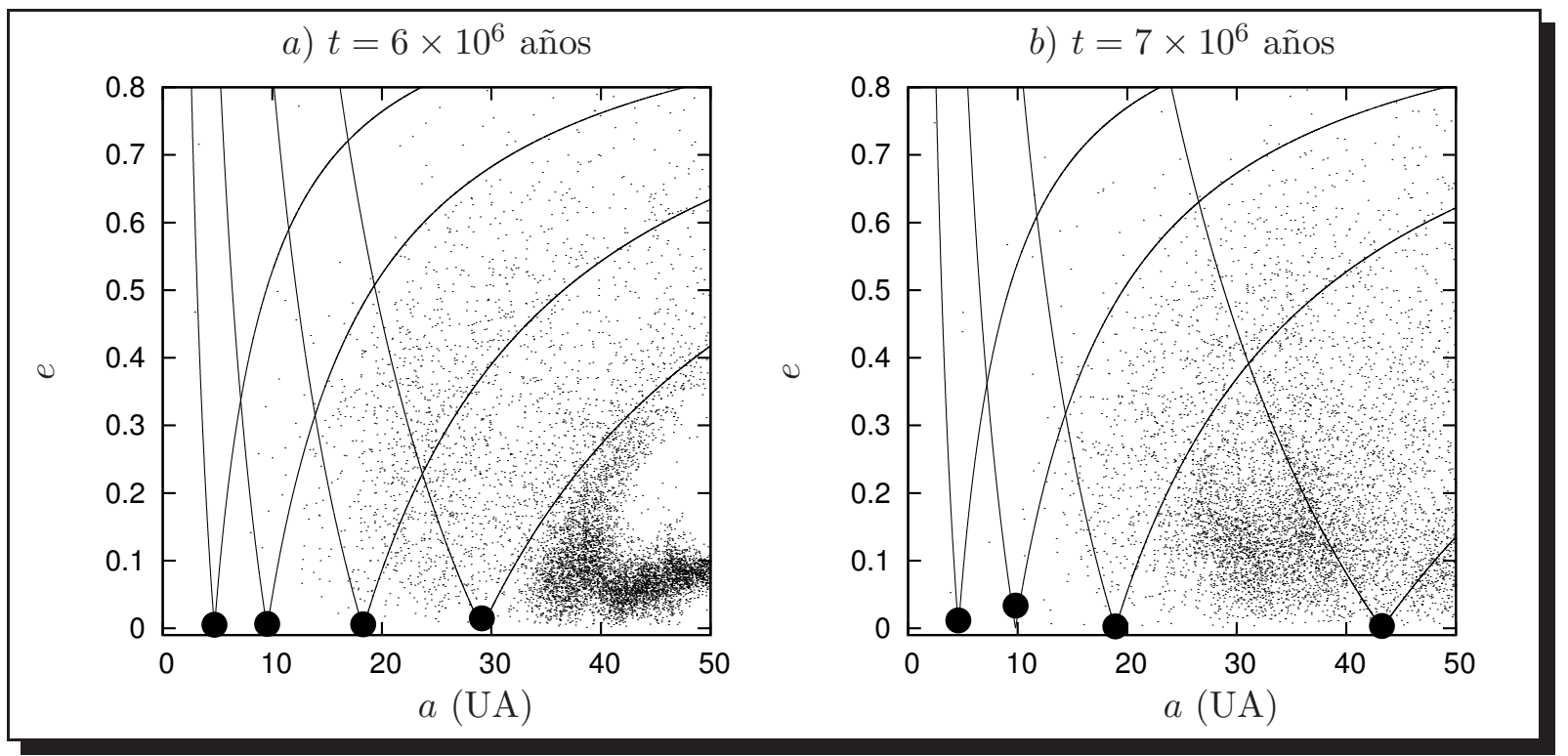

Figura 3.7. Estado dinámico del disco en el plano $(a, e)$, de semiejes $a$ y excentricidades $e$, antes y después de que Neptuno alcance el borde exterior del disco y revierta la dirección de su migración. Las líneas continuas indican los bordes de las respectivas zonas de cruce de cada planeta.

$6 \times 10^{6}$ años, Neptuno acelera rápidamente su migración y alcanza el borde exterior del disco donde revierte su migración, para luego, al tiempo $1.2 \times 10^{7}$ años, comenzar a migrar nuevamente hacia el exterior. Finalmente, la figura 3.7 muestra, en el plano de semiejes y excentricidades, el aumento del estado de excitación de la zona exterior del disco durante el primer pasaje de Neptuno hacia el borde exterior y su reversión de la dirección de migración.

\subsection{El modelo de Niza.}

Los modelos descritos en la sección anterior suponen que Neptuno migra hacia el borde exterior del disco sobre una órbita cuasi-circular. Existe, sin embargo, una propuesta alternativa donde la evolución orbital de los planetas gigantes es sustancialmente diferente. Este modelo, conocido como modelo de Niza (debido a que los investigadores involucrados trabajaban en el Observatorio de la Costa Azul en tal ciudad de Francia al momento de su concepción) exhibe una corta, pero remarcable, fase de inestabilidad dinámica global producto del cruce de Saturno y Júpiter en una resonancia de movimiento medio de bajo orden. Tal escenario permite, de acuerdo a una serie de simulaciones numéricas en los trabajos que presentan dicho modelo, $(i)$ reproducir los elementos orbitales actuales de los planetas gigantes (Tsiganis y otros, 2005), (ii) mostrar que es posible la captura de las poblaciones de troyanos de Júpiter y Neptuno (Morbidelli y otros, 2005; Tsiganis y otros, 2005), (iii) dar una explicación al denominado bombardeo intenso tardío sobre los planetas interiores (Gomes y otros, 2005) y, finalmente, $(i v)$ reproducir las principales características de la región transneptuniana (Levison y otros, 2008). 
El modelo de Niza postula que los planetas gigantes se encuentran inicialmente en órbitas cuasi circulares $\left(e \sim 10^{-3}\right)$ y coplanares $\left(i<0.1^{\circ}\right)$ con una configuración en las separaciones orbitales significativamente más compacta que la actual. Específicamente, los planetas gigantes gaseosos son ubicados de manera que Júpiter orbita a 5.45 UA (esto es, levemente más alejado del Sol que en la actualidad), mientras que Saturno es colocado entre 8 y 9 UA, siempre dentro de la resonancia exterior de movimientos medios 1:2 de Júpiter (a una localización de $8.65 \mathrm{UA}$ ), la cual es una hipótesis clave para el modelo ${ }^{8}$. Por su parte, los planetas gigantes helados, son dispuestos con Urano entre 11 y 13 UA y Neptuno entre 13.5 y 17 UA. La otra pieza clave del modelo es la presencia de un disco de planetesimales más allá de los planetas conteniendo, en total, unas $35 M_{\oplus}$ y extendiéndose hasta su borde externo a unas 30 UA (como lo sugiere el trabajo de Gomes y otros (2004), reseñado en la sección anterior) y con el borde interno aproximadamente 1.5 UA del planeta más alejado, valor que es impuesto por el requisito de que las partículas de este disco tienen que tener un tiempo de vida dinámico del orden o mayor al tiempo de vida supuesto para el disco gaseoso, unos $3 \times 10^{6}$ años, pues de lo contrario ellas habrían sido eliminadas durante la etapa en que el gas estaba presente.

Con tal configuración, las simulaciones muestran que los planetesimales en las cercanías del borde interno del disco son dispersados por Neptuno, muchos de ellos hacia la región interior, sobre una escala de tiempo del orden de los millones de años de manera que la interacción gravitacional de tales planetesimales con los planetas causa una lenta migración de los mismos, moviéndose Júpiter hacia afuera y los restantes planetas hacia el interior. La clave del modelo de Niza es que después de un largo tiempo, entre 305 y 1100 millones de años, Júpiter y Saturno, al migrar en direcciones opuestas, cruzan su mutua resonancia 1:2 causando un comportamiento caótico sobre las órbitas de los gigantes helados que desestabilizan todo el disco de planetesimales. En efecto, el cruce de tal resonancia excita las excentricidades de Júpiter y Saturno a valores levemente mayores que los actuales. Sin embargo, este evento conduce a un aumento significativo de las excentricidades de Urano y Neptuno favoreciéndose así, en un corto período de tiempo, los encuentros entre ambos y, en consecuencia, un comportamiento caótico de sus órbitas ${ }^{9}$. Esto lleva a ambos gigantes helados a migrar rápidamente hacia afuera en órbitas de gran excentricidad $(e \sim 0.3-0.4)$, las cuales penetran profundamente en el disco de planetesimales, desestabilizándolo y dispersando planetesimales del mismo sobre todo el sistema. A continuación, en unos millones de años, las excentricidades de Urano y Neptuno y, en menor medida, las de Júpiter y Saturno, son reducidas producto de la fricción dinámica ejercida por el disco de planetesimales, y los planetas alcanzan sus órbitas finales cuando Neptuno, en su rápida migración que produce el vaciamiento del disco, llega a las cercanías del borde externo del mismo. Los valores finales de semiejes mayores, excentricidades e inclinaciones de las simulaciones reproducen cuantitativamente las órbitas actuales de los planetas gigantes.

Por otra parte, los planetesimales dispersados hacia el interior del sistema durante la desestabilización del disco, junto con material proveniente del cinturón de asteroides

\footnotetext{
${ }^{8}$ En la actualidad Saturno se encuentra en el exterior de la resonancia externa 1:2 de Júpiter y en el interior de la resonancia externa 2:5.

${ }^{9}$ De hecho, en la mitad de las simulaciones llevadas a cabo por Tsiganis y otros (2005), se observa que los gigantes helados intercambian sus órbitas.
} 
también desestabilizado por la migración de Júpiter y Saturno que los aleja de la resonancia 1:2, son considerados, por el modelo, los causantes del denominado bombardeo intenso tardío: un corto período de tiempo, aproximadamente unos 700 millones de años después de la formación del sistema solar hace unos $4.6 \times 10^{9}$ años atrás, donde la intensidad de impactores sobre los cuerpos del sistema solar interior fue de gran magnitud ${ }^{10}$.

Además, las simulaciones muestran que una fracción de las partículas dispersas son atrapadas en la resonancia de movimientos medios 1:1 de Júpiter, dando origen, cuando Júpiter y Saturno están lo suficiente alejados de la resonancia mutua 1:2, a una población estable cuya distribución orbital resulta estar de acuerdo con la de los troyanos de Júpiter. Las simulaciones muestran también una población de troyanos de Neptuno capturados cuando la excentricidad del planeta está siendo amortiguada por el disco de planetesimales.

Finalmente, la región transneptuniana, de acuerdo al modelo, es formada a partir de las partículas del disco original dispersadas hacia afuera durante la evolución orbital de Neptuno desde una órbita de gran excentricidad hasta la amortiguación de la misma. Las simulaciones permiten reproducir las dos clases dinámicas, "fría" y "caliente", de la población del cinturón de Kuiper clásico, su límite exterior en la resonancia de movimientos medios 1:2 con Neptuno, las poblaciones resonantes con Neptuno y el déficit de objetos sobre órbitas con baja excentricidad entre 45 y 48 UA. Más aún, el modelo da cuenta de una población de objetos más allá de la resonancia 1:2 con Neptuno con distancias perihélicas de unas 40 UA, las cuales se corresponden a la actual población de objetos del disco dispersado.

El modelo de Niza, sin embargo, produce una población fría del cinturón de Kuiper clásico con una excentricidad media más alta que la observada y no puede reproducir las más altas inclinaciones presentes en el cinturón clásico. Pero la principal debilidad del modelo de Niza es que la configuración inicial de los planetas es escogida sin tener en cuenta la historia previa de la evolución planetaria cuando el disco de gas estaba aún presente. Este problema es abordado por Morbidelli y otros (2007) y conduce a una variante en la cual los planetas se encuentran en una configuración multiresonante luego de la desaparición del disco gaseoso, con Júpiter y Saturno en su mutua resonancia de movimientos medios 2:3 y Urano y Neptuno en la respectiva resonancia 3:4. La presencia de un disco de planetesimales por fuera de la región planetaria rompe eventualmente la configuración resonante originándose una fase de inestabilidad similar al modelo original pero ahora cuando Júpiter y Saturno cruzan su mutua resonancia 5:3. Este escenario refinado del modelo anterior es conocido como modelo de Niza 2.

Con fines de testear nuestro código, consideraremos una simulación compatible con el escenario del modelo de Niza planteado en el trabajo de Gomes y otros (2005) para dar una explicación del bombardeo intenso tardío. Específicamente, los cuatro planetas gigantes son colocados sobre el plano del disco, siendo los semiejes iniciales de Júpiter, Saturno, Urano y Neptuno, 5.45, 8.18, 11.5 y 14.2 UA, respectivamente, con excentri-

\footnotetext{
${ }^{10}$ La hipótesis del bombardeo tardío resulta de la interpretación de los registros geológicos de las rocas lunares traídas por las misiones Apolo y meteoritos lunares encontrados en la Tierra (Tera y colaboradores, 1974). Sin embargo, no hay un acuerdo unánime sobre el mismo e incluso su existencia es puesta en duda por otros investigadores (Armitage, 2010).
} 


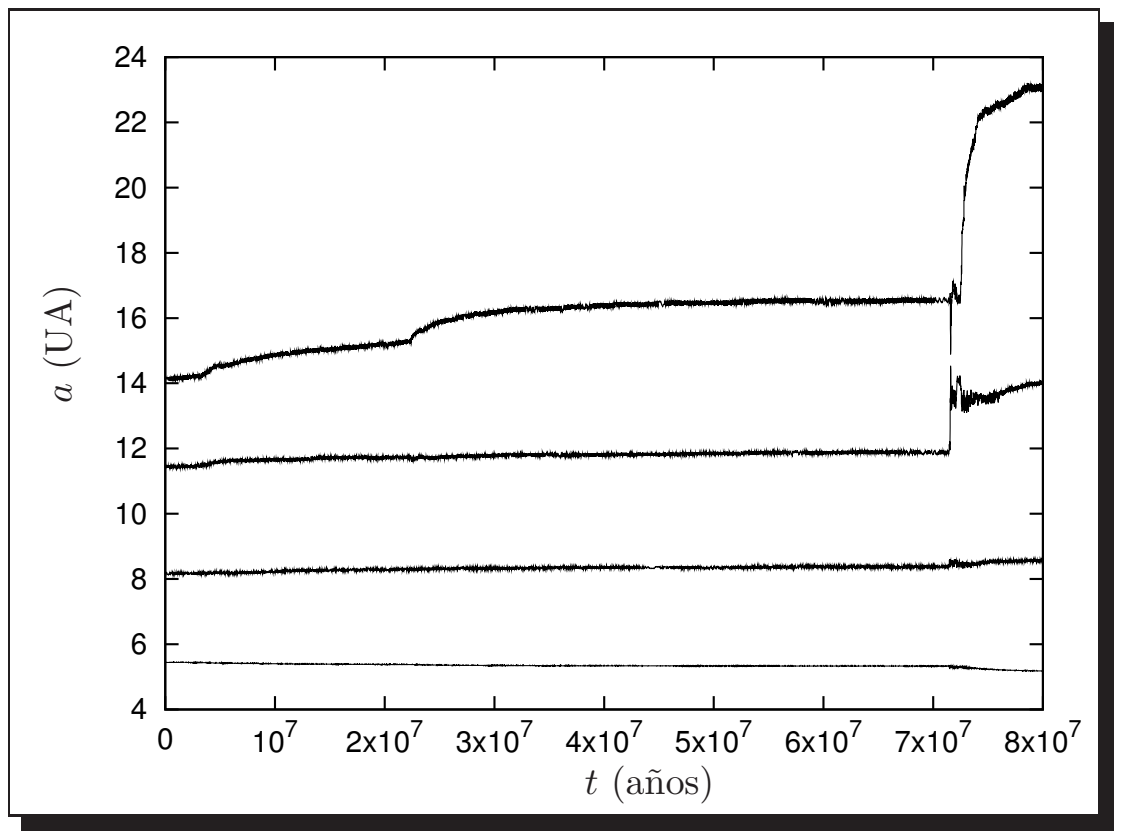

Figura 3.8. Evolución temporal de los semiejes mayores de los planetas gigantes en una simulación del modelo de Niza. Las curvas se corresponde, de abajo hacia arriba, a Júpiter, Saturno, Urano y Neptuno.

cidades e inclinaciones nulas. El disco de planetesimales se extiende originalmente más allá de Neptuno, desde 15.5 UA hasta 34 UA, con un perfil de densidad que decae con la distancia radial sobre el plano del disco como $r^{-1}$ y tiene una masa total de $35 M_{\oplus}$. El disco se supone inicialmente "frío", con las partículas en órbitas cuasi-circulares, con una dispersión de las excentricidades de $10^{-3}$ y una dispersión de las inclinaciones de $0.5 \times 10^{-3}$. El disco es simulado utilizando $N=5000$ partículas de igual masa. La simulación procede sobre un intervalo temporal de $80 \times 10^{6}$ años con un paso de integración $\Delta t=0.25$ años. Planetesimales que adquieren órbitas hiperbólicas o cuyas distancias heliocéntricas sean mayores que 1000 UA son eliminados del sistema. Como en todas las simulaciones de este capítulo, las partículas del disco se suponen no interactuantes, pero éstas tienen masa y por lo tanto influyen sobre el movimiento de los planetas gigantes (y viceversa). La Fig. 3.8 muestra la evolución de los semiejes de los planetas gigantes. En nuestra simulación el cruce de la resonancia 1:2 mutua de Júpiter y Saturno se produce entre $t=72 \times 10^{6}$ y $t=73 \times 10^{6}$ años. Nótese que, en tal evento, Neptuno y Urano sufren un encuentro cercano. La diferencia de un orden de magnitud en el tiempo del cruce de la resonancia en nuestra simulación respecto al trabajo mencionado se debe, como también indican los autores, a la sensibilidad de dicho instante con la localización inicial del borde interno del disco de planetesimales respecto al planeta más alejado: cuanto menor sea esta distancia más rápidamente se produce el evento. La Fig. 3.9 muestra el estado de excitación del sistema, planetas y disco. Como puede verse, por un lado, el cruce de la resonancia origina una fase transitoria de altas excentricidades en los planetas gigantes helados, y por otro lado, la migración de Neptuno hacia el interior del disco produce una alta dispersión de sus planetesimales. 


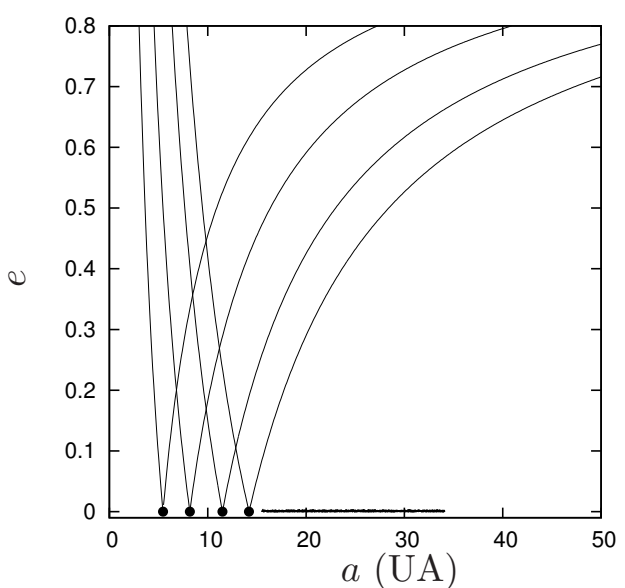

(a) $t=0$ años (comienzo de la simulación).

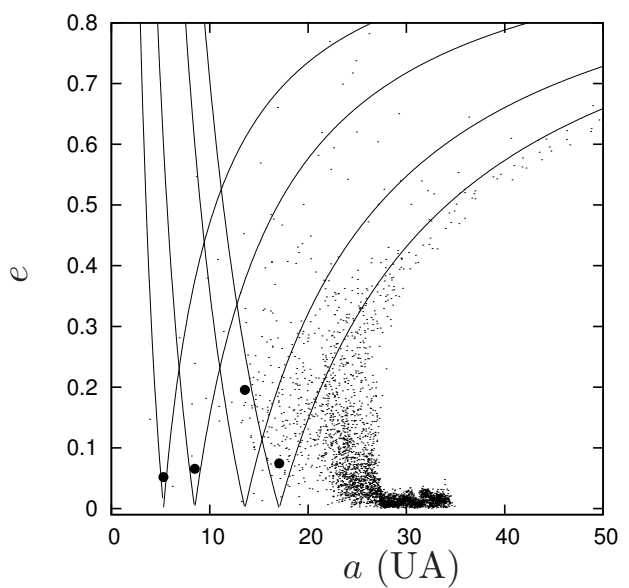

(c) $t=7.2 \times 10^{7}$ (al comienzo del cruce de la resonancia 1:2).

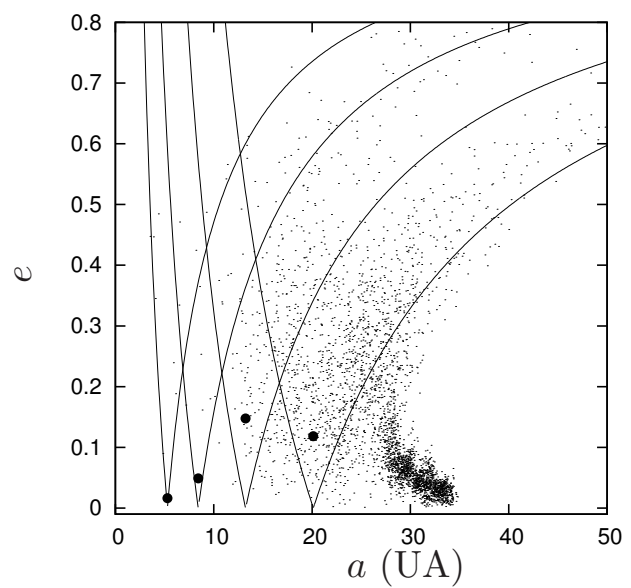

(e) $t=7.3 \times 10^{7}$ (al final del cruce de la resonancia $1: 2$ ).

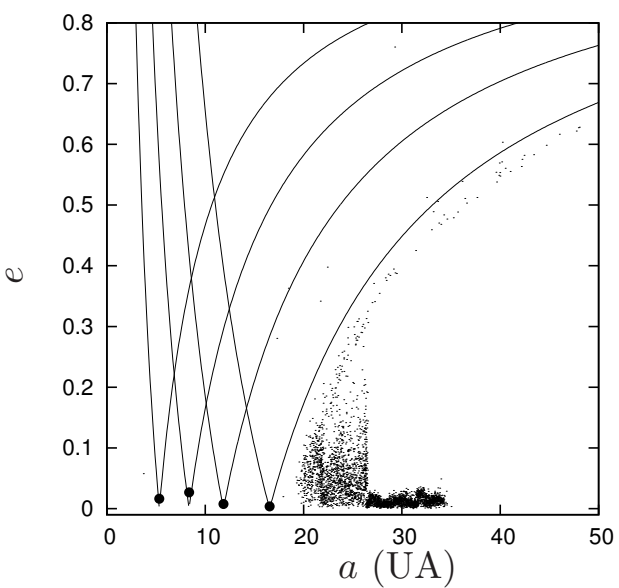

(b) $t=7 \times 10^{7}$ (previo al cruce de la resonancia $1: 2$ ).

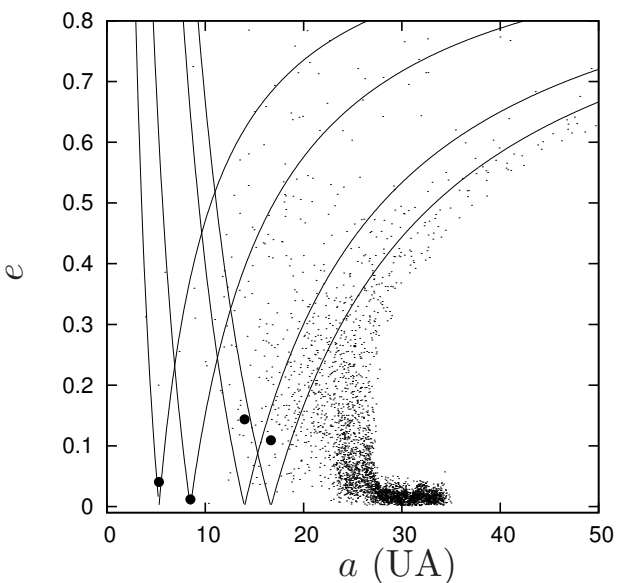

(d) $t=7.25 \times 10^{7}$ (durante el cruce de la resonancia 1:2).

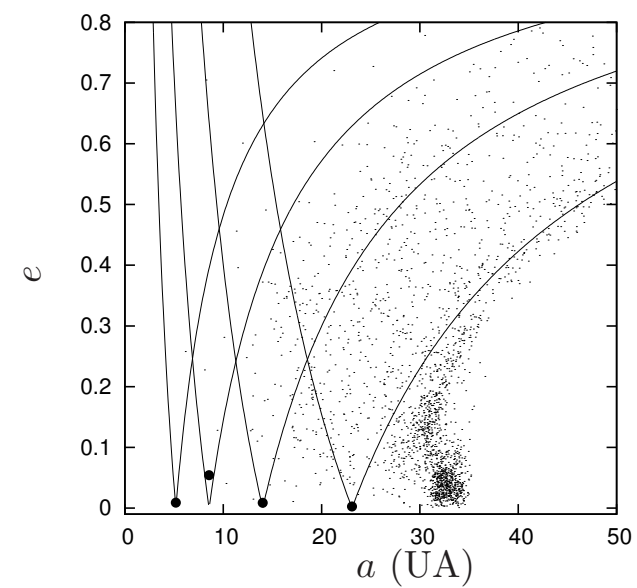

(f) $t=8 \times 10^{7}$ (final de la simulación).

Figura 3.9. Estado dinámico del sistema en el plano $(a, e)$, de semiejes $a$ y excentricidades $e$, en diversos instantes de una simulación del modelo de Niza. Las líneas continuas indican los bordes de las respectivas zonas de cruce de cada planeta gigante. 


\section{Capítulo 4}

\section{La importancia del número de partículas en las simulaciones de la interacción planeta-disco}

\subsection{Introducción.}

Un punto clave de las simulaciones de la interacción planeta-disco es el número de partículas utilizadas para describir el disco de planetesimales. Conforme se considera en las simulaciones una masa cada vez más pequeña del planeta, más partículas son requeridas para obtener resultados confiables en un disco de una masa dada. Así, si el disco no es simulado con suficiente partículas, la interacción planeta-disco será dominada por la fuerte interacción resultante de encuentros entre el planeta y "partículas" cuyas masas son demasiado grandes. El propósito de este capítulo es examinar la sensibilidad de las simulaciones con el número de partículas que modelan al disco.

\subsection{Planteo del problema.}

Para determinar la sensibilidad de las simulaciones con el número de partículas utilizado recurrimos a una serie de simulaciones numéricas del escenario interacción planeta-disco descrito por Cioncio y Brunini (2002), escenario que hemos presentado en el capítulo 3.

Esencialmente, nuestro modelo consiste en un planeta de masa $M$, ubicado inicialmente a 5 UA del cuerpo central, el Sol, sobre una órbita circular e inmerso en un disco de masa $M_{\text {disco }}$ constituido por $N$ planetesimales de igual masa $m$. Específicamente, se consideran cinco valores para la masa del cuerpo planetario, a saber: $M=10,8,6,4$ y $2 M_{\oplus}$. Por otra parte el disco de planetesimales se toma con una masa $M_{\text {disco }} \simeq 160 M_{\oplus}$ con una densidad superficial constante de $12 \mathrm{~g} \mathrm{~cm}^{-2}$, valor consistente con un modelo de nebulosa solar de masa mínima $\left(0.01 M_{\odot}\right)$. El límite interno del disco es tomado a 2 UA, mientras que el límite externo es colocado a 12.5 UA, cubriendo así todas las resonancias relevantes. Las excentricidades e inclinaciones de las órbitas iniciales de las 
partículas se consideran con una distribución normal de media cero (restringida al rango no negativo en el caso de la excentricidad) y dispersiones $\left\langle e^{2}\right\rangle^{1 / 2}=2\left\langle i^{2}\right\rangle^{1 / 2}=10^{-2}$. Mientras que la interacción planeta-planetesimal es calculada plenamente, la autointeracción del disco es ignorada. Por otra parte, las colisiones con el planeta son tratadas como procesos de acreción perfecta y planetesimales que alcanzan órbitas hiperbólicas son eliminados del sistema. Destaquemos también que, en nuestras simulaciones, ninguna partícula colisiona con el Sol, de hecho sólo un número insignificante $(\leq 2)$ alcanza una distancia heliocéntrica menor que $1 \mathrm{UA}$. El paso de integración escogido es $\Delta t=0.1$ años $\left(\leq 5 \times 10^{-2}\right.$ del menor período orbital en cada simulación). Este paso, resultante de una serie de experimentos previos, permite un tiempo de cómputo razonable y, a la vez, una adecuada detección de los encuentros.

\subsection{Sensibilidad al número de partículas.}

Los resultados más relevantes de las simulaciones son la tasa de variación del semieje mayor del planeta, $\dot{a}$, la cual determina un tiempo característico de deriva $\tau$ dado por la ecuación (3-5) esto es, el tiempo necesario para que el cambio en el semieje sea del mismo orden que su valor; y el torque $\Gamma$ ejercido por el disco sobre el planeta, dado por la ecuación (3-6).

Como muestran Cionco y Brunini (2002), para el disco simulado la interacción planeta-disco debería resultar en una migración hacia el interior del disco, con una tasa de migración a ser comparada con las predicciones de la teoría lineal de las ondas de densidad, la cual, como hemos indicado en el capítulo 3, constituye una teoría representativa de la fricción dinámica en discos. Para determinar la sensibilidad de la tasa de migración con respecto al número $N$ de planetesimales efectuamos diversas simulaciones con el disco de masa total dada, pero modelado con diferente número de partículas, a saber, en el rango de 1000 - 250000 , para cada masa planetaria $M$. De esta forma, en total, tenemos 16 simulaciones para las cinco masas planetarias consideradas. Cuando la "granularidad" de la simulación, definida por la relación $\mathrm{M} / \mathrm{m}$, es menor que un cierto valor crítico, esperamos que el efecto de la fricción dinámica resonante entre las partículas del disco y el planeta sea completamente superada por los efectos estocásticos de los encuentros cercanos, conduciendo así a un modelo incorrecto del problema. En tanto que, cuando el número de partículas es incrementado, el "ruido" introducido por la granularidad del modelo es reducido y esperamos que la acción de la fricción dinámica resulte relevante, obteniéndose una tasa de migración apropiada. Tal situación es ejemplificada dramáticamente en la Figura 4.1. La misma ilustra la evolución temporal del semieje mayor para el caso de un planeta de $M=2 M_{\oplus}$ inicialmente ubicado a $5 \mathrm{UA}$ en el disco simulado con tres diferentes valores para el número $N$ de planetesimales. En la primera simulación $N=50000$, con lo cual la masa de cada planetesimal es $m=0.00327 M_{\oplus}(\mathrm{y} M / m \simeq 610)$. En la segunda simulación, $N=150000$ y $m=0.00109 M_{\oplus}(M / m \simeq 1830)$. Mientras que en la última, $N=250000, m=0.00066 M_{\oplus}(M / m \simeq 3050)$. Estos resultados muestran claramente que los modelos del disco de la primera y la segunda simulación no son confiables, mientras que recién la tercera simulación muestra una migración hacia el interior del disco como lo requiere nuestro escenario. Nótese también que la figura ilus- 


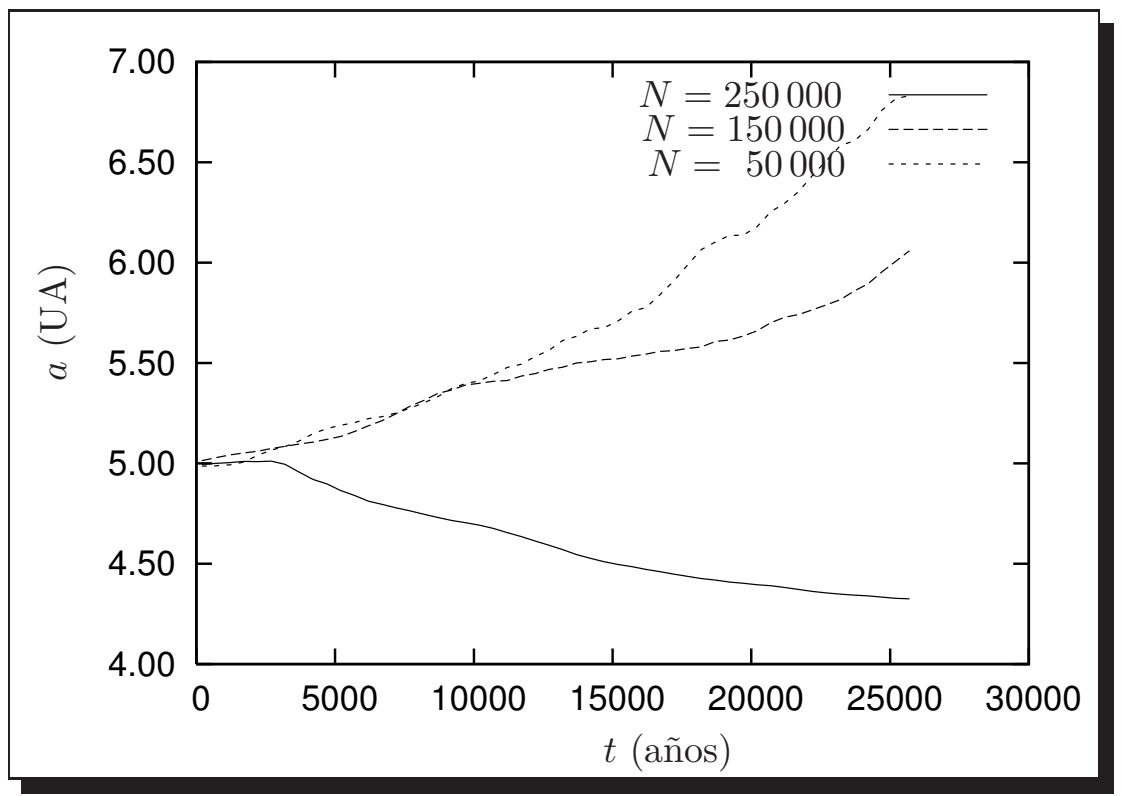

Figura 4.1. Evolución temporal del semieje mayor en tres simulaciones para un planeta de masa $M=2 M_{\oplus}$.

tra un resultado potencialmente peligroso: aun cuando la interacción dinámica con el disco sea pobremente modelada el semieje mayor del planeta puede experimentar una suave migración, aunque en la dirección incorrecta.

\subsection{El término de fricción dinámica y su correcto modelado.}

Para verificar que las simulaciones incorrectas son efectivamente consecuencia de un incorrecto modelado de la fricción dinámica, recurrimos a la descripción estadística de las órbitas de los planetesimales conocida como aproximación de "partícula en una caja" (Stewart y Wetherill, 1988). Este modelo concibe a los planetesimales en órbitas aproximadamente circulares coplanares y concéntricas. La mutua interacción gravitacional perturba sus órbitas conduciendo a valores pequeños (pero no despreciables) de sus excentricidades e inclinaciones. La aproximación estadística ignora las órbitas individuales y utiliza, en su lugar, una distribución de densidad de probabilidad para describir la distribución de los elementos orbitales de la población de planetesimales. Suponiendo que los perihelios y las longitudes de los nodos están orientados al azar, la distribución de excentricidades e inclinaciones de la población puede ser descrita por una única variable, la velocidad media cuadrática, $v$, con respecto a una órbita de referencia local circular y de inclinación nula:

$$
v=\left(\frac{5}{8} e^{2}+\frac{1}{2} i^{2}\right)^{\frac{1}{2}} v_{K},
$$

siendo $v_{K}$ la velocidad circular kepleriana local, e la excentricidad e $i$ la inclinación (medida en radianes). En un disco kepleriano los cuerpos con distintos semiejes orbitan 
el cuerpo central con diferentes velocidades medias, siendo éstas menores cuanto mayor sea la distancia al cuerpo central. Existe, así, un movimiento ordenado en el disco. Ahora bien, la velocidad $v$ de un planetesimal relativa a la de una órbita circular local de inclinación nula, puede considerarse como una magnitud esencialmente aleatoria. Tales velocidades aleatorias juegan un papel análogo a las velocidades aleatorias de las moléculas de un gas confinado en una cavidad a una temperatura dada, y de allí el nombre de "partícula en una caja" (particle-in-a-box) con que se designa al modelo estadístico.

Distintos mecanismos pueden afectar la evolución de la velocidad aleatoria. Una ecuación que describe la evolución de un cuerpo testigo de masa $M$ interactuando con la población de cuerpos de masa $m$ es dada por Stewart y Wetherrill (1988). En nuestro disco de partículas, libre de gas, esta ecuación consta de cuatro términos. Específicamente, la tasa de cambio de $V$, la velocidad relativa media cuadrática del cuerpo de masa $M$, está dada por

$$
\frac{d V}{d t}=A+B+C+D
$$

donde los cuatro términos se identifican como sigue:

A. Excitación de la velocidad causada por encuentros gravitacionales,

$$
A \propto(M+m) V^{2}+\left(m v^{2}-M V^{2}\right) .
$$

$B$. Excitación de la velocidad originada por colisiones inelásticas,

$$
B \propto m\left(V^{2}-v^{2}\right)+2 M V^{2} .
$$

C. Amortiguamiento de la velocidad debido a la disipación de energía por colisiones inelásticas,

$$
C \propto-\left[m\left(V^{2}-v^{2}\right)+2 M V^{2}\right]
$$

D. Transferencia de energía de los cuerpos mayores a los más pequeños por fricción dinámica,

$$
D \propto m v^{2}-M V^{2}
$$

Los factores de proporcionalidad en estas ecuaciones son dados en el apéndice F. Aquí, los términos $A$ y $B$ se comportan como términos viscosos o resistivos, donde las interacciones mutuas entre planetesimales transforman la energía del flujo medio en movimiento aleatorio, tratando de llevar al sistema a un estado en el cual las velocidades son independientes de la masa. En un disco rotando uniformemente ambos términos serían nulos. Por su parte, el término $C$, con origen en las colisiones inelásticas, desexcita al sistema. Finalmente, el término $D$, que es el término de fricción dinámica que nos interesa, trata de llevar al sistema a un estado de equipartición de energía donde las velocidades de los cuerpos más pequeños se incrementan mientras que las de los cuerpos más grandes disminuyen. En efecto, si $V \approx v$ y $M>m$ se sigue de la ecuación (4-6) para $D$ que $\mathrm{d} V / \mathrm{d} t<0$ por acción de este término e, invirtiendo el papel de 
las masas, es claro que $\mathrm{d} v / \mathrm{d} t>0$. Si la fricción dinámica opera eficientemente llevando al sistema un estado de equipartición de energía entre los cuerpos mayores y menores, el tiempo necesario para que esto ocurra se denomina tiempo de relajación del sistema. Nótese, sin embargo, que para un disco de planetesimales con una amplia distribución de tamaños, el estado de equipartición nunca es alcanzado debido a la presencia de los términos viscosos que operan en sentido contrario.

Es importante dejar en claro que el modelo anterior constituye una descripción correcta del problema sólo localmente. En consecuencia, para computar los cuatro términos involucrados en la tasa de cambio de la velocidad relativa $V$ del planeta $M$ embebido en el disco, debemos tomar en cuenta sólo aquellos planetesimales que se encuentren dentro de una región anular centrada a la distancia del planeta, $a-\Delta r<r<a+\Delta r$, de cierto ancho $\Delta r=\alpha R_{\mathrm{H}}$, siendo

$$
R_{\mathrm{H}}=a\left(\frac{M}{3 M_{\odot}}\right)^{1 / 3},
$$

el radio de Hill del planeta y, típicamente, tomamos $\alpha=3$.

La evaluación de los términos para las tres simulaciones correspondientes a $M=$ $2 M_{\oplus}$, descritas en la sección anterior, muestran, en primer lugar, que el término $C$ no afecta fuertemente la evolución de la velocidad. Así, los términos $A+B$ y $D$ controlan el comportamiento de la tasa de cambio de $V$. Si la fricción dinámica es relevante, entonces el término $D$ debe tomar el control sobre el término $A+B$ y por lo tanto esperamos que la razón $|A+B| /|D|$ permanezca menor que la unidad a lo largo del tiempo. La Figura 4.2 muestra las contribuciones relativas de los términos $A+B$ y $D$ en las tres simulaciones y permiten confirmar que sólo en la tercera simulación $(N=250000)$ la acción de la fricción dinámica es relevante. De hecho, para la primera $(N=50000)$ y la segunda simulación $(N=150000)$, la condición $|A+B|>|D|$ es satisfecha un $12 \%$ sobre la duración de la misma, mientras que en la tercer simulación tal relación sólo se satisface un $4 \%$.

\subsection{Simulaciones confiables y el número de partí- culas.}

La Tabla 4.1 y la Figura 4.3 presentan el número de planetesimales necesarios que hemos encontrado para obtener resultados confiables en nuestro conjunto de simulaciones. Como indican Cionco y Brunini (2002), la tasa de migración $\dot{a}$ es determinada por un ajuste de mínimos cuadrados sobre la evolución temporal del semieje mayor $a$ en un intervalo donde la variación del mismo sea monótona (donde efectos como relajación o saturación de resonancias están ausentes). A partir de tal valor, el tiempo de deriva $\tau$ es calculado de acuerdo a la ecuación (3-5) y, en virtud de la circularización de la órbita del cuerpo planetario por acción de la fricción dinámica, es lícito aplicar la ecuación (3-6) para estimar el torque $\Gamma$ que ejerce el disco sobre el mismo.

Como ya hemos mencionado, para nuestras condiciones particulares del disco, se espera una migración planetaria hacia el interior del disco. En consecuencia, el término 


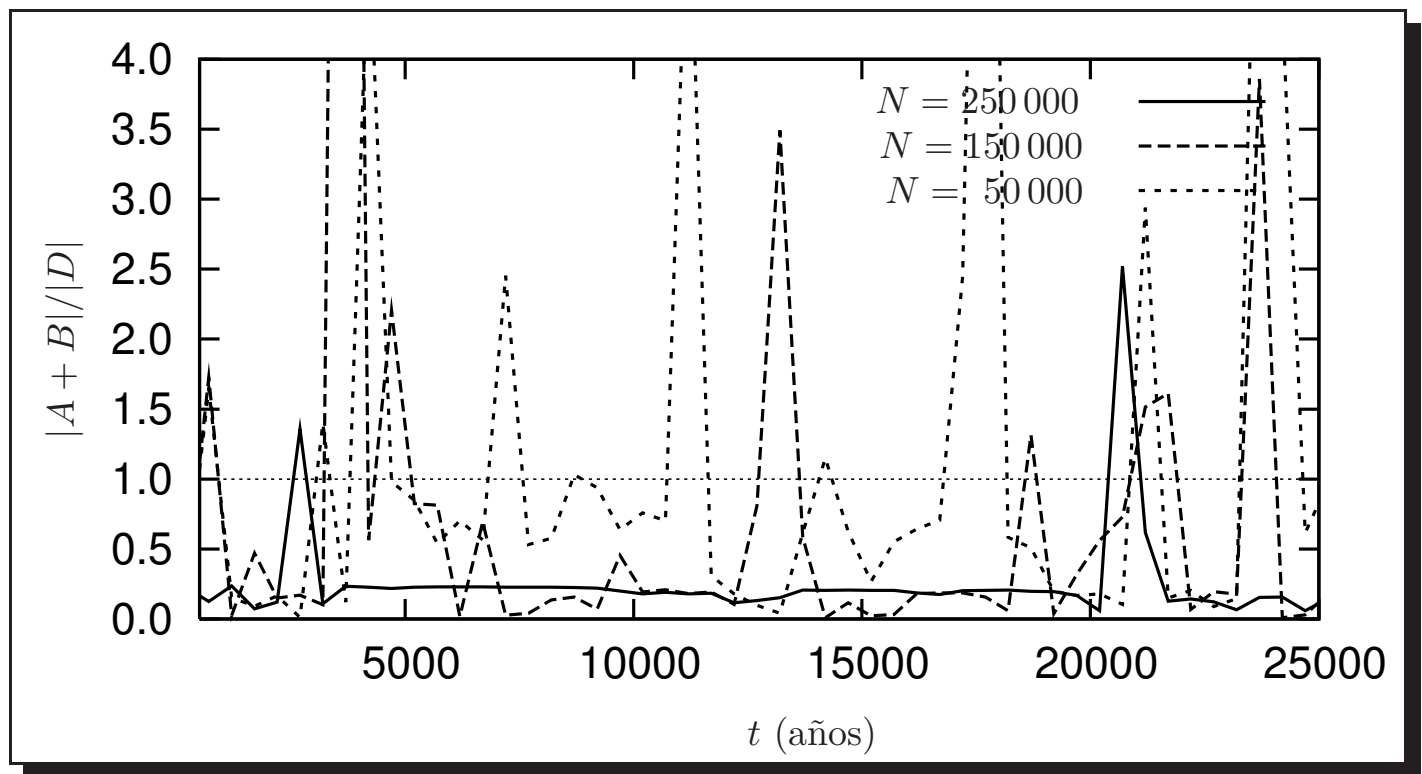

Figura 4.2. Contribución de los términos $A+B$ y $D$ en las simulaciones con $N=50000, N=150000$ y $N=250000$ partículas para el disco de planetesimales en el caso de un cuerpo planetario de $M=2 M_{\oplus}$.

de fricción dinámica debería ser

$$
m v^{2}-M V^{2}<0
$$

En un disco de planetesimales, podemos suponer que las velocidades aleatorias dependen de la masa en la forma (Safronov, 1969)

$$
v=\left(\frac{m}{M}\right)^{q} V,
$$

donde, si la fricción dinámica opera el exponente $q$ es negativo, e igual a $-\frac{1}{2}$ si la equipartición de la energía es completamente alcanzada (Wetherill y Stewart, 1989). Combinando estas dos ecuaciones, y usando el hecho de que todos los discos de nuestras simulaciones tienen la misma masa total, encontramos que el número de planetesimales debería ser

$$
N>M_{\text {disco }} / M
$$

Un ajuste de mínimos cuadrados a nuestras simulaciones confiables, muestra, tal como se observa en la Figura 4.3, que el número adecuado para obtener resultados confiables varía como $M^{-1.3}$, un resultado compatible con la predicción anterior.

\subsection{Relevancia de los resultados.}

La eficiencia de nuestro código nos permitió realizar simulaciones con un gran número de planetesimales y explorar así la importancia de usar muchas partículas en las simulaciones de la interacción planeta-disco. Nuestros resultados muestran que utilizar un número de planetesimales menor que cierto valor puede conducir a resultados 


\begin{tabular}{cccccc}
\hline$M$ & $N$ & $m$ & $\dot{a}$ & $\tau$ & $\Gamma$ \\
\hline 10 & 20000 & 0.00830 & $-3.22 \times 10^{-6}$ & $1.55 \times 10^{6}$ & $-1.31 \times 10^{-9}$ \\
8 & 50000 & 0.00327 & $-5.42 \times 10^{-6}$ & $9.22 \times 10^{5}$ & $-1.85 \times 10^{-10}$ \\
6 & 50000 & 0.00327 & $-7.72 \times 10^{-6}$ & $6.48 \times 10^{5}$ & $-1.97 \times 10^{-10}$ \\
4 & 100000 & 0.00082 & $-5.35 \times 10^{-6}$ & $9.36 \times 10^{5}$ & $-9.12 \times 10^{-11}$ \\
2 & 250000 & 0.00065 & $-1.04 \times 10^{-5}$ & $4.82 \times 10^{5}$ & $-9.84 \times 10^{-11}$ \\
\hline
\end{tabular}

Tabla 4.1. Simulaciones confiables en nuestro experimentos. La masa $M$ del planeta y la masa individual $m$ de las partículas del disco son expresadas en masas terrestres. La tasa de migración $\dot{a}$ es expresada en unidades de $\mathrm{UA}$ año ${ }^{-1}$, el tiempo de deriva $\tau$ en años y el torque ejercido sobre el planeta en $M_{\odot} \mathrm{AU}^{2}$ año ${ }^{-2}$.

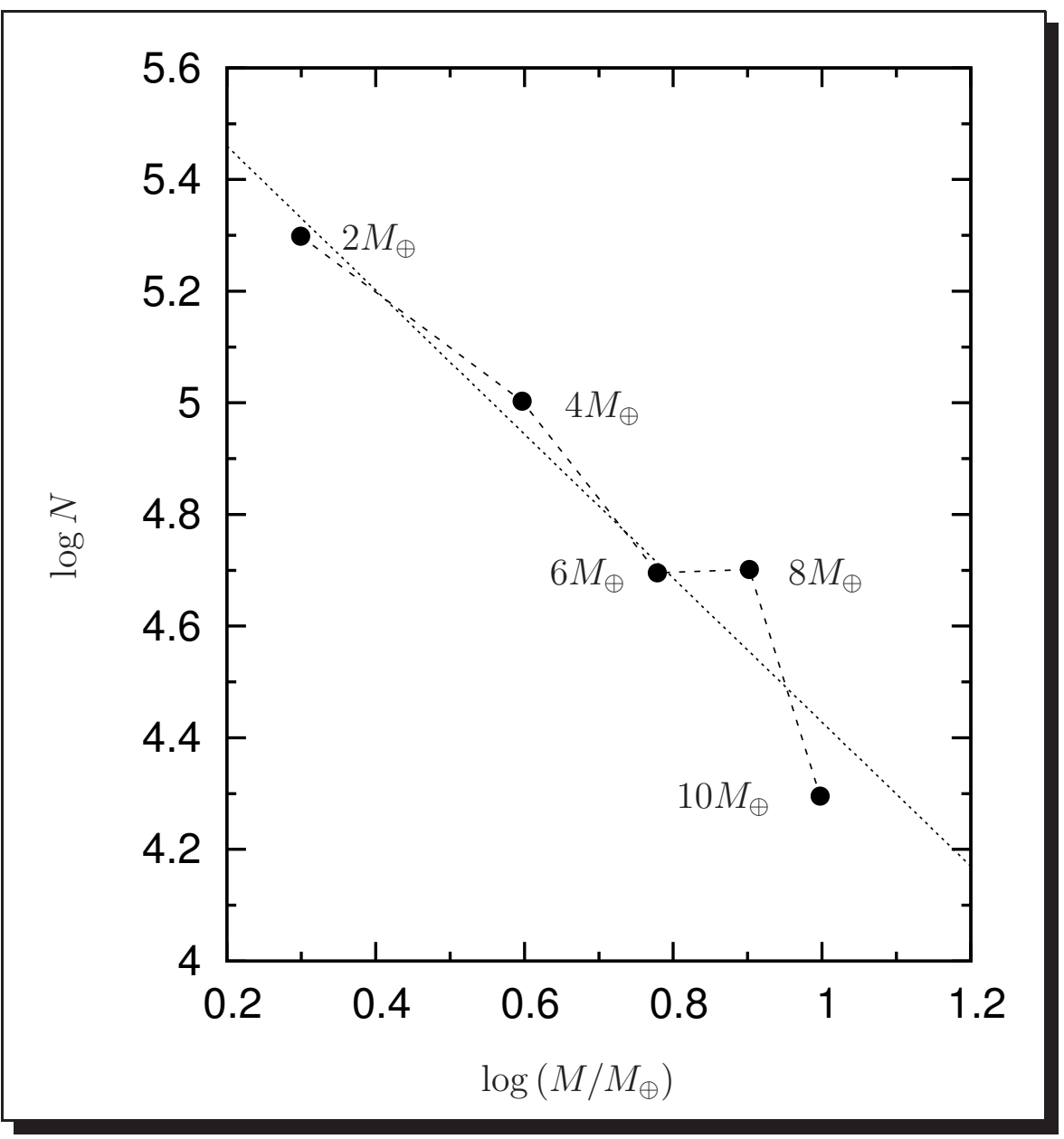

Figura 4.3. Número de partículas que dan resultados confiables para diferentes masas planetarias (expresadas en masas terrestres). 
incorrectos. Como indicamos, esta situación es potencialmente peligrosa, debido a que, como lo muestra la Fig. 4.1, aun si la interacción dinámica con el disco es pobremente modelada, el semieje mayor del planeta puede experimentar una suave migración en la dirección incorrecta.

Aunque los resultados han sido obtenidos con un disco de estructura particular (densidad superficial constante), una posible estrategia a considerar en una simulación con un disco de una dada estructura, sería efectuar una serie de simulaciones de prueba con un planeta masivo de masa $M_{0}$ tal que requiera de un número relativamente pequeño $N_{0}$ de planetesimales (lo cual implica un tiempo de cómputo razonablemente rápido). Una vez que es determinado el valor mínimo $N_{0}$ que da una dirección y tasa de migración confiables, el número de partículas $N$ que debe ser usado en la simulación de interés, con la masa planetaria $M$, puede ser determinado de la relación

$$
N \geq N_{0}\left(\frac{M_{0}}{M}\right)^{1.3}
$$




\section{Capítulo 5}

\section{El efecto de la autointeracción del disco de planetesimales}

\subsection{Introducción.}

Como hemos mencionado en varias oportunidades a lo largo de la tesis, en un escenario con la presencia de un planeta embebido en el disco, el grado de exitación del disco de planetesimales es gobernado fundamentalmente por la interacción gravitatoria de tal planeta con las partículas del disco, siendo la autointeracción del disco un efecto secundario que típicamente es despreciado en las simulaciones numéricas debido al alto costo de tiempo de cómputo involucrado. Esta simplificación implica la pérdida de ciertos procesos que ocurren al tomar en cuenta la presencia de la autointeracción. En particular, si el disco está dinámicamente excitado en una localización resonante, tal excitación, bajo ciertas condiciones, se propaga como una onda a través del disco, amortiguando así la propia excitación (Ward y Hahn, 1998, 2003). Sin embargo, tal propagación de onda es posible sólo si la excitación del disco es muy pequeña $(e<0.01$, $i<0.3^{\circ}$, Hahn, 2003) y por lo tanto, en un escenario con un planeta embebido en el disco interactuando fuertemente con las partículas que se encuentran dentro de su zona de cruce, el efecto descrito no influye significativamente en la migración del planeta. Sin embargo, la autointeracción modifica colectivamente el estado dinámico de tales partículas y por lo tanto es de esperar efectos secundarios. Por otra parte, al despreciar la autointeracción no se está tomando en cuenta la amortiguación de la excitación dinámica del disco producto de las posibles colisiones entre partículas. Goldreich y otros (2004) muestra un sistema en el cual la amortiguación colisional es tan eficiente que toda excitación en el disco debido a la presencia de un planeta es casi completamente disipada instantáneamente. Sin embargo, para que esto suceda, se requiere de una distribución de tamaños de las partículas del disco mas bien extrema, escencialmente todos los planetesimales deben tener un diámetro del orden de un centímetro. A continuación, exploraremos nuestros propios resultados sobre los efectos de la autointeracción del disco en las localizaciones resonantes de un planeta.

Como consecuencia de la migración orbital de un planeta los planetesimales del disco pueden ser capturados en las localizaciones resonantes en movimiento medio del planeta (las cuales se mueven con él). En el marco del modelo de aproximación 


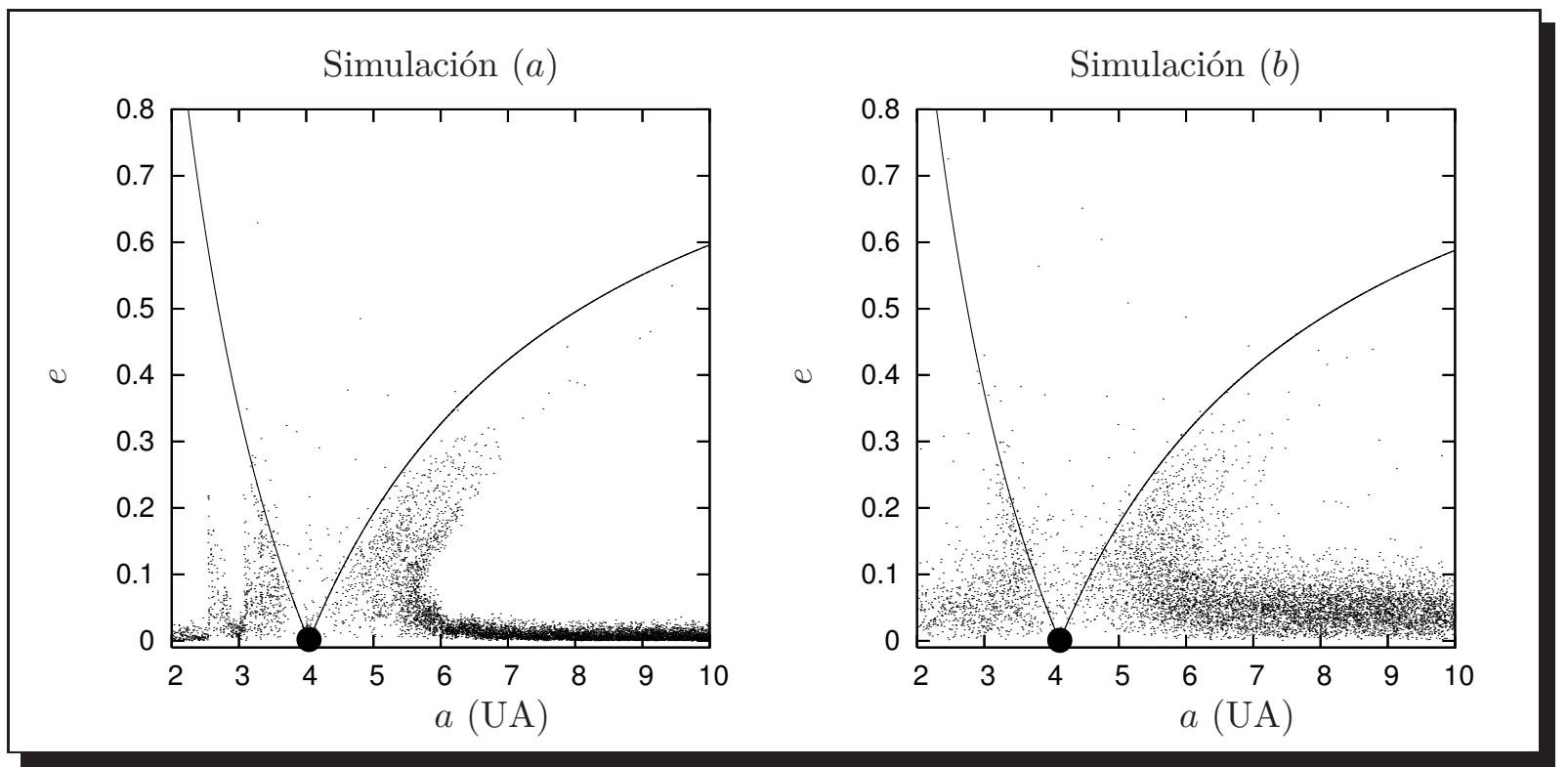

Figura 5.1. Estado dinámico del sistema en el plano $(a, e)$, de semiejes mayores $a$ y excentricidades $e$, a $t=10^{5}$ años para un cuerpo de masa $M=10 M_{\oplus}$ migrando en un disco de planetesimales de $M_{\text {disco }}=200 M_{\oplus}$ en una simulación ( $a$ ) donde la autointeracción del disco es ignorada y $(b)$ donde es considerada. La simulación $(a)$ da cuenta de dos poblaciones capturadas en las resonancias de movimientos medios internas 2:1 y 3:2 del planeta, en las localizaciones nominales, al tiempo considerado, 2.52 UA y $3.05 \mathrm{UA}$, respectivamente.

adiabática del proceso, si el planeta se mueve hacia el interior (exterior) del disco, la captura en resonancia puede ocurrir sólo en las resonancias interiores (exteriores) y en tanto la excentricidad inicial $e$ del planetesimal sea menor que cierto valor crítico $e_{\text {crít }}$. Para excentricidades mayores la captura es un evento probabilístico cuya probabilidad decrece conforme $e$ es mayor. Así, el proceso de captura en resonancia es dependiente del estado de excitación dinámica de la región del disco que encuentra el planeta, la cual será diferente si se tiene en cuenta, o no, la autointeracción del disco.

Para cuantificar la situación consideremos nuevamente un escenario de migración del trabajo de Cionco y Brunini (2002) descrito en el la sección 3.2 del capítulo 3 y base de los resultados del capítulo anterior. Específicamente, un planeta de masa $M=10 M_{\oplus}$ es ubicado inicialmente a 5 UA del cuerpo central, el Sol, sobre una órbita circular e inmerso en un disco de planetesimales de masa $M_{\text {disco }}=200 M_{\oplus}$ con densidad superficial constante, que se extiende inicialmente entre 2 y $12.5 \mathrm{UA}$, siendo modelado con $N=10000$ partículas de igual masa $m$. La Figura 5.1 muestra el estado dinámico del sistema en el plano de semiejes mayores $a$ y excentricidades $e$ transcurridos $t=10^{5}$ años, ilustrando la figura de la izquierda, $(a)$, una simulación donde la autointeracción del disco no es considerada, en tanto que la figura de la derecha (b) corresponde a una simulación donde sí es contemplada. Como podemos apreciar, la localización final del planeta es similar y, de hecho, las tasas de migración calculadas son del mismo orden para ambas simulaciones ${ }^{1}$. Sin embargo, en la simulación $(a)$ es clara la presencia de dos

\footnotetext{
${ }^{1}(a) \dot{a}=-6.15 \times 10^{-6} \mathrm{UA} \mathrm{año}^{-1},(b) \dot{a}=-5.97 \times 10^{-6} \mathrm{UA} \mathrm{año}^{-1}$.
} 
poblaciones capturadas en las resonancias de movimientos medios internas $2: 1$ y $3: 2$, no distinguibles en la simulación (b). Además la región más externa del disco, no sensible a la perturbación del planeta migrando hacia el interior, se encuentra claramente en un estado de excitación mayor en la simulación (b) debido a la autointeracción no contemplada en $(a)$. El problema se debe a que $N=10000$ partículas para simular el disco resulta suficiente para que la "granularidad" $M / m$ sea adecuada para modelar la migración del planeta, pero, para dicho valor, la masa individual de cada partícula es $m=0.02 M_{\oplus}$, esto es dos veces la masa de la Luna $\left(0.01 M_{\oplus}\right)$. Si bien no podemos excluir la presencia de cuerpos con masas de este orden en los discos protoplanetarios cuando los planetas ya están formados, ellos sólo contribuyen con una pequeña fracción de la masa total del disco, el cual, por lo tanto, está compuesto de un mayor número de cuerpos en el rango de masas menores. Así, la masa de cada partícula individual del disco utilizada en la simulación no es realista, y aunque modela correctamente la migración, no da cuenta de las poblaciones resonantes al considerar autointeracción.

Nos interesa, pues, en este capítulo, determinar cuál debe ser el número de partículas $N$ para modelar un disco de planetesimales donde la autointeracción es considerada y que, a la vez, sea consistente con la posibilidad de captura en resonancia durante la migración planetaria. Para ello deduciremos un conjunto de ecuaciones generales que permitan describir el estado de autoexcitación del disco, en el marco de un modelo local muy simplificado, las cuales aplicaremos a nuestro problema.

\subsection{Resonancias de movimiento medio y captura en resonancia.}

En esta sección introducimos brevemente la terminología general utilizada para describir el fenómeno de resonancias de movimiento medio así como las ecuaciones básicas del proceso de captura en resonancia. Nuestra discusión está basada en el Capítulo 8, Resonant Perturbations, del libro Solar System Dynamics de Murray y Dermott (1999).

En esencia, una resonancia de movimientos medios ocurre cuando la razón de los períodos orbitales de dos cuerpos son conmensurables, es decir, es aproximadamente igual a la razón de dos enteros pequeños. Consideremos en primer lugar las resonancias que introduce un cuerpo de masa planetaria $M$ al perturbar el movimiento de un planetesimal de masa $m$ en una órbita interior a $M$. Si despreciamos las (lentas) frecuencias de precesión de los nodos y pericentros de las órbitas, una resonancia de movimientos medios ocurre cuando para dos enteros particulares $j_{1}$ y $j_{2}$ se satisface la relación:

$$
j_{1} n_{p}+j_{2} n=0
$$

donde $n$ y $n_{p}$ son las frecuencias o movimientos medios de las órbitas keplerianas del planetesimal y el planeta, respectivamente. Tomando $j_{1}=p+q, j_{2}=-p$ donde $p$ y $q$ son enteros positivos, podemos escribir la conmensurabilidad de movimientos medios involucrada como

$$
\frac{n}{n_{p}}=\frac{p+q}{p}
$$


y decimos que el planetesimal $m$ se encuentra en una resonancia interna $(p+q): p$ de $M$, siendo $q$ el orden de la resonancia. Esta conmensurabilidad de movimientos medios implica, a su vez, una relación entre los semiejes mayores $a$ y $a_{p}$ de las órbitas de $m$ y $M$. Suponiendo que tanto la masa del cuerpo planetario como la del planetesimal son despreciables frente a la del cuerpo central $\left(m, M \ll m_{0}\right)$, se sigue de la tercera ley de Kepler:

$$
n^{2} a^{3}=G m_{0}, \quad n_{p}^{2} a^{3}=G m_{0},
$$

y por lo tanto

$$
\frac{a}{a_{p}}=\left(\frac{n_{p}}{n}\right)^{2 / 3}=\left(\frac{p}{p+q}\right)^{2 / 3} .
$$

Debido a la dependencia con el semieje mayor $a_{p}$, las resonancias de movimientos medios están espacialmente localizadas. Definimos la localización nominal $a_{n}$ de la resonancia $(p+q): p$ como el valor del semieje mayor del cuerpo $m$ que satisface la relación:

$$
a_{n}=\left(\frac{p}{p+q}\right)^{2 / 3} a_{p} .
$$

Consideremos ahora la situación en que el cuerpo $M$ perturba a un planetesimal de masa $m$ en una órbita exterior al mismo. De manera similar a lo anterior, una resonancia ocurre si existen valores enteros $j_{1}$ y $j_{2}$ tales que

$$
j_{1} n+j_{2} n_{p}=0
$$

Tomando nuevamente $j_{1}=p+q, j_{2}=-p$, la relación de conmensurabilidad entre los movimientos medios puede escribirse como

$$
\frac{n}{n_{p}}=\frac{p}{p+q}
$$

y decimos que el planetesimal $m$ se encuentra en una resonancia externa $p:(p+q)$ de $M$, siendo $q$ el orden de la resonancia. Asimismo, la relación entre semiejes que esta relación implica permite definir la localización nominal $a_{n}$ de la resonancia externa $p:(p+q)$ como el valor del semieje mayor del cuerpo $m$ que satisface la relación:

$$
a_{n}=\left(\frac{p+q}{p}\right)^{2 / 3} a_{p} .
$$

En nuestro problema trataremos solamente con resonancias de primer orden. En tal caso $q=1$, de manera que $j_{1}=p+1, j_{2}=-p$. Siguiendo a Murray y Dermott (1999), escribimos:

$$
j_{1}=p+1 \equiv j, \quad j_{2}=-p \equiv 1-j
$$

De esta forma, para una resonancia interna de primer orden $j:(j-1)$ la relación de conmensurabilidad y localización nominal de la misma son escritas como

$$
\frac{n}{n_{p}}=\frac{j}{j-1}, \quad a_{n}=\left(\frac{j-1}{j}\right)^{2 / 3} a_{p} .
$$


En tanto que, para una resonancia externa de primer orden $(j-1): j$,

$$
\frac{n}{n_{p}}=\frac{j-1}{j}, \quad a_{n}=\left(\frac{j}{j-1}\right)^{2 / 3} a_{p} .
$$

Consideremos ahora las ecuaciones básicas del proceso de captura en resonancias de primer orden. En primer lugar, supongamos que el planetesimal de masa $m$ se encuentra en una resonancia de primer orden interna $j:(j-1)$ del cuerpo de masa planetaria $M$ moviéndose, pues, en una órbita interior a $M$. Además se considera que ambos cuerpos se mueven sobre un mismo plano y que la órbita del planeta es circular y permanece sin perturbar (con lo cual $m \ll M$ ). Así pues, la órbita de $m$ será capturada en resonancia si su excentricidad $e \leq e_{\text {crít }}$, siendo (Murray y Dermott, 1999)

$$
e_{\text {crít }}=\sqrt{6}\left[\frac{3}{\left|f_{d}\right|}(j-1)^{4 / 3} j^{2 / 3} \frac{m_{0}}{M}\right]^{-1 / 3} \text {. }
$$

El factor $f_{d} \equiv f_{d}(\alpha)$ está asociado con la parte directa de la función perturbadora del problema perturbativo de dos cuerpos y depende, para una dada resonancia, solamente de la razón de semiejes $\alpha=a / a_{p}$. Su expresión, obtenida de la primera entrada de la Tabla 8.1 de Murray y Dermott (1999), es

$$
f_{d}(\alpha)=\frac{1}{2}[-2 j-\alpha D] b_{1 / 2}^{(j)}(\alpha)
$$

En esta ecuación $D$ es el operador diferencial $D \equiv \mathrm{d} / \mathrm{d} \alpha$ y $b_{1 / 2}^{(j)}(\alpha)$ es un coeficiente de Laplace. En forma general, el coeficiente de Laplace $b_{s}^{(j)}(\alpha)$, donde $s=i+1 / 2$ es un semientero $(s=1 / 2,3 / 2,5 / 2, \ldots)$ es definido como

$$
\frac{1}{2} b_{s}^{(j)}(\alpha)=\frac{1}{2 \pi} \int_{0}^{2 \pi} \frac{\cos (j \psi)}{\left(1-2 \alpha \cos \psi+\alpha^{2}\right)^{s}} \mathrm{~d} \psi
$$

En forma alternativa, estos coeficientes puede ser expresados como una serie convergente para todo $\alpha<1^{2}$ :

$$
\begin{aligned}
\frac{1}{2} b_{s}^{(j)}(\alpha)= & \frac{s(s+1) \ldots(s+j-1)}{1 \cdot 2 \cdot 3 \cdots j} \alpha^{j} \times \\
& {\left[1+\frac{s(s+j)}{1(j+1)} \alpha^{2}+\frac{s(s+1)(s+j)(s+j+1)}{1 \cdot 2(j+1)(j+2)} \alpha^{4}+\ldots\right] . }
\end{aligned}
$$

En el caso particular $j=0$ el factor fuera de los corchetes es igual a la unidad. Tales coeficientes satisfacen ciertas relaciones entre sí, en particular:

$$
\begin{gathered}
b_{s}^{(-j)}=b_{s}^{(j)}, \\
D b_{s}^{(j)}=s\left(b_{s+1}^{(j-1)}-2 \alpha b_{s+1}^{(j)}+b_{s+1}^{(j)}\right) .
\end{gathered}
$$

\footnotetext{
${ }^{2}$ Recuérdese que como estamos tratando una resonancia interna $a<a_{p}$, la condición $\alpha<1$ se satisface.
} 
Utilizando la relación (5-16b) es inmediato mostrar que la expresión de $f_{d}$ dada por la ecuación (5-13) puede escribirse como:

$$
f_{d}(\alpha)=-j b_{1 / 2}^{(j)}(\alpha)-\frac{1}{4} \alpha\left[b_{3 / 2}^{(j-1)}(\alpha)-2 \alpha b_{3 / 2}^{(j)}(\alpha)+b_{3 / 2}^{(j+1)}(\alpha)\right] .
$$

En efecto,

$$
\begin{aligned}
f_{d}(\alpha) & =\frac{1}{2}[-2 j-\alpha D] b_{1 / 2}^{(j)} \\
& =\frac{1}{2}\left[-2 j b_{1 / 2}^{(j)}-\alpha D b_{1 / 2}^{(j)}\right] \\
& =\frac{1}{2}\left[-2 j b_{1 / 2}^{(j)}-\alpha \frac{1}{2}\left(b_{3 / 2}^{(j-1)}-2 \alpha b_{3 / 2}^{(j)}+b_{3 / 2}^{(j+1)}\right)\right] \\
& =-j b_{1 / 2}^{(j)}-\frac{1}{4} \alpha\left[b_{3 / 2}^{(j-1)}-2 \alpha b_{3 / 2}^{(j)}+b_{3 / 2}^{(j+1)}\right] .
\end{aligned}
$$

la cual es, precisamente, la ecuación requerida.

En la evaluación de $f_{d}$ para una resonancia particular el valor de $\alpha$ queda determinado por la localización nominal de la resonancia. Así, en nuestro caso, según la ecuación (5-10):

$$
\alpha=\frac{a_{n}}{a_{p}}=\left(\frac{j-1}{j}\right)^{2 / 3} .
$$

Las ecuaciones presentadas permiten, en particular, determinar el valor crítico de la excentricidad para la captura en las resonancias 2:1 y 3:2 como función de la razón de la masa del cuerpo planetario a la del cuerpo central.

\section{Resonancia interna 2:1.}

$$
e_{\text {crit }}^{(2: 1)}=1.543056\left(\frac{M}{m_{0}}\right)^{1 / 3} \text {. }
$$

En efecto, en este caso $j=2$, con lo cual, según la ecuación (5-17),

$$
f_{d}(\alpha)=-2 b_{1 / 2}^{(2)}(\alpha)-\frac{1}{4} \alpha\left[b_{3 / 2}^{(1)}(\alpha)-2 \alpha b_{3 / 2}^{(2)}(\alpha)+b_{3 / 2}^{(3)}(\alpha)\right] .
$$

Tomando

$$
\alpha=\left(\frac{1}{2}\right)^{2 / 3}=0.629961
$$

la evaluación numérica de los coeficientes de Laplace conduce a

$$
\begin{aligned}
& b_{1 / 2}^{(2)}=0.365314, \\
& b_{3 / 2}^{(1)}=4.92256, \\
& b_{3 / 2}^{(2)}=3.65544, \\
& b_{3 / 2}^{(3)}=2.60296,
\end{aligned}
$$

con lo cual,

$$
f_{d}=-1.190492 .
$$

La ecuación (5-12) conduce, entonces, al resultado planteado. 


\section{Resonancia interna 3:2.}

$$
e_{\text {crít }}^{(3: 2)}=1.237014\left(\frac{M}{m_{0}}\right)^{1 / 3}
$$

En efecto, en este caso $j=3$, con lo cual, según la ecuación (5-17),

$$
f_{d}(\alpha)=-3 b_{1 / 2}^{(3)}(\alpha)-\frac{1}{4} \alpha\left[b_{3 / 2}^{(2)}(\alpha)-2 \alpha b_{3 / 2}^{(3)}(\alpha)+b_{3 / 2}^{(4)}(\alpha)\right] .
$$

Tomando

$$
\alpha=\left(\frac{2}{3}\right)^{2 / 3}=0.76343
$$

la evaluación numérica de los coeficientes de Laplace conduce a

$$
\begin{aligned}
& b_{1 / 2}^{(3)}=0.400516, \\
& b_{3 / 2}^{(2)}=10.4857, \\
& b_{3 / 2}^{(3)}=8.8484, \\
& b_{3 / 2}^{(4)}=7.33676,
\end{aligned}
$$

con lo cual,

$$
f_{d}=-2.025222 \text {. }
$$

La ecuación (5-12) conduce, nuevamente, al resultado planteado.

Consideremos ahora la situación en que un planetesimal de masa $m$ se encuentra en una resonancia de primer orden externa $(j-1): j$ del cuerpo de masa planetaria $M$ al moverse en una órbita exterior a $M$. En este caso, la órbita de $m$ será capturada en resonancia si su excentricidad $e \leq e_{\text {crít }}$, siendo

$$
e_{\text {crít }}=\sqrt{6}\left[\frac{3}{\left|f_{d}\right|} j^{2} \frac{m_{0}}{M}\right]^{-1 / 3} .
$$

Como antes, el factor $f_{d} \equiv f_{d}(\alpha)$ depende, para una dada resonancia, solamente de la razón de semiejes $\alpha=a_{p} / a<1$. Su expresión, obtenida de la segunda entrada de la Tabla 8.1 de Murray y Dermott (1999), es

$$
f_{d}(\alpha)=\frac{1}{2}[-1+2 j+\alpha D] b_{1 / 2}^{(j-1)}(\alpha) .
$$

Utilizando la relación (5-16b) que satisfacen los coeficientes de Laplace, obtenemos su expresión explícita:

$$
f_{d}(\alpha)=\frac{1}{2}(2 j-1) b_{1 / 2}^{(j-1)}(\alpha)-\frac{1}{4} \alpha\left[b_{3 / 2}^{(j-2)}(\alpha)-2 \alpha b_{3 / 2}^{(j-1)}(\alpha)+b_{3 / 2}^{(j)}(\alpha)\right] .
$$

En efecto,

$$
\begin{aligned}
f_{d}(\alpha) & =\frac{1}{2}[-1+2 j+\alpha D] b_{1 / 2}^{(j-1)}(\alpha) \\
& =\frac{1}{2}\left[-b_{1 / 2}^{(j-1)}+2 j b_{1 / 2}^{(j-1)}+\alpha D b_{1 / 2}^{(j-1)}(\alpha)\right] \\
& =\frac{1}{2}\left[-b_{1 / 2}^{(j-1)}+2 j b_{1 / 2}^{(j-1)}+\alpha \frac{1}{2}\left(b_{3 / 2}^{(j-2)}-2 \alpha b_{3 / 2}^{(j-1)}+b_{3 / 2}^{(j)}\right)\right] \\
& =\frac{1}{2}(2 j-1) b_{1 / 2}^{(j-1)}-\frac{1}{4} \alpha\left[b_{3 / 2}^{(j-2)}(\alpha)-2 \alpha b_{3 / 2}^{(j-1)}(\alpha)+b_{3 / 2}^{(j)}(\alpha)\right] .
\end{aligned}
$$


la cual es la ecuación planteada.

El valor de $\alpha$ requerido para evaluar $f_{d}$ en una resonancia particular resulta de considerar la localización nominal de la resonancia. Así, según la ecuación (5-11):

$$
\alpha=\frac{a_{p}}{a_{n}}=\left(\frac{j-1}{j}\right)^{2 / 3} .
$$

En particular, expresemos el valor crítico de la excentricidad para la captura en las resonancias 1:2 y 2:3 como función de la razón de la masa del cuerpo planetario a la del cuerpo central.

\section{Resonancia externa 1:2.}

$$
e_{\text {crít }}^{(1: 2)}=1.273990\left(\frac{M}{m_{0}}\right)^{1 / 3} .
$$

En efecto, en este caso $j=2$, con lo cual, según la ecuación (5-23),

$$
f_{d}(\alpha)=\frac{3}{2} b_{1 / 2}^{(2)}(\alpha)-\frac{1}{4} \alpha\left[b_{3 / 2}^{(0)}(\alpha)-2 \alpha b_{3 / 2}^{(1)}(\alpha)+b_{3 / 2}^{(2)}(\alpha)\right] .
$$

Tomando

$$
\alpha=\left(\frac{1}{2}\right)^{2 / 3}=0.629961,
$$

la evaluación numérica de los coeficientes de Laplace conduce a

$$
\begin{aligned}
& b_{1 / 2}^{(1)}=0.75684, \\
& b_{3 / 2}^{(0)}=6.05826, \\
& b_{3 / 2}^{(1)}=4.92256, \\
& b_{3 / 2}^{(2)}=3.65544,
\end{aligned}
$$

con lo cual,

$$
f_{d}=1.688312 .
$$

La ecuación (5-21) conduce, entonces, al resultado planteado.

\section{Resonancia externa 2:3.}

$$
e_{\text {crit }}^{(2: 3)}=1.105788\left(\frac{M}{m_{0}}\right)^{1 / 3} .
$$

En efecto, en este caso $j=3$, con lo cual, según la ecuación (5-23),

$$
f_{d}(\alpha)=\frac{5}{2} b_{1 / 2}^{(2)}(\alpha)-\frac{1}{4} \alpha\left[b_{3 / 2}^{(1)}(\alpha)-2 \alpha b_{3 / 2}^{(2)}(\alpha)+b_{3 / 2}^{(3)}(\alpha)\right] .
$$


Tomando

$$
\alpha=\left(\frac{2}{3}\right)^{2 / 3}=0.76343,
$$

la evaluación numérica de los coeficientes de Laplace conduce a

$$
\begin{aligned}
b_{1 / 2}^{(2)} & =0.61745, \\
b_{3 / 2}^{(1)} & =12.08476, \\
b_{3 / 2}^{(2)} & =10.4857, \\
b_{3 / 2}^{(3)} & =8.8484,
\end{aligned}
$$

con lo cual,

$$
f_{d}=2.484005 \text {. }
$$

La ecuación (5-21) conduce, así, al resultado planteado.

\subsection{Autoexcitación del disco de planetesimales.}

En esta sección nos interesa describir la evolución de la excentricidad de los planetesimales de un disco producto de la interacción con otros planetesimales del mismo.

Consideremos, a una dada distancia $r=a$ sobre el plano del disco, un planetesimal de masa $m$ cuya órbita inicial es próxima a una órbita circular. Cada interacción con otro planetesimal introduce un pequeño cambio en la velocidad. Suponiendo que estas perturbaciones están orientadas al azar, la magnitud del cambio puede ser obtenida considerando su valor cuadrático medio $\Delta v^{2} \equiv\left\langle(\Delta \mathbf{v})^{2}\right\rangle$. Utilizando la ecuaciones de Gauss para la variación de los elementos orbitales (ver, por ejemplo, Danby, 1992) resulta, al promediar sobre la órbita, que el valor cuadrático medio del cambio de velocidad está relacionado con el cambio cuadrático medio $\Delta e^{2} \equiv\left\langle(\Delta e)^{2}\right\rangle$ de la excentricidad del planetesimal por la ecuación

$$
\Delta e^{2}=\frac{5}{6} \frac{\Delta v^{2}}{v_{\mathrm{K}}^{2}}
$$

donde $v_{\mathrm{K}}$ es la velocidad kepleriana circular local.

En efecto, en el marco del tratamiento perturbativo del problema de los dos cuerpos, la acción de los otros planetesimales puede ser considerada como una perturbación sobre la órbita kepleriana inicialmente circular y describirse, entonces, por una fuerza perturbadora $\mathbf{F}$ por unidad de masa (dimensionalmente, una aceleración). Descomponiendo $\mathbf{F}$ según las direcciones dirigidas a lo largo del radio vector $\left(\hat{u}_{r}\right)$, transversal a éste $\left(\hat{u}_{\theta}\right)$ y perpendicular al plano de la órbita no perturbada $\left(\hat{u}_{A}\right)$

$$
\mathbf{F}=R \hat{u}_{r}+B \hat{u}_{\theta}+N \hat{u}_{A},
$$

la ecuación de Gauss del problema perturbativo correspondiente a la variación de la excentricidad de la órbita (Danby, 1992) es

$$
\frac{\mathrm{d} e}{\mathrm{~d} t}=\frac{n a^{2}}{\mu} \sqrt{1-e^{2}}[R \operatorname{sen} \nu+B(\cos \nu+\cos E)],
$$


siendo $\mu=G m_{0}$ (donde $G$ es la constante de gravitación universal y $m_{0}$ la masa del cuerpo central y suponemos que $\left.m \ll m_{0}\right), n=\sqrt{\mu / a^{3}}$ el movimiento medio de la órbita kepleriana de semieje mayor $a$, y $\nu$ y $E$ la anomalía media y excéntrica, respectivamente, del planetesimal a un dado instante de tiempo en su órbita. En el caso de una órbita aproximadamente circular, $r \simeq a \mathrm{y} e \simeq 0$, la ecuación anterior se reduce a

$$
\frac{\mathrm{d} e}{\mathrm{~d} t}=\frac{1}{v_{\mathrm{K}}}(R \operatorname{sen} \nu+2 B \cos \nu),
$$

siendo $v_{\mathrm{K}}=n a=\sqrt{\mu / a}$ la velocidad kepleriana circular local en $r=a$. Cada perturbación $\mathbf{F}$ introduce un pequeño cambio en la velocidad $\Delta \mathbf{v}=\mathbf{F} \Delta t$. Suponiendo que tales perturbaciones están orientadas aleatoriamente, el cambio vectorial total en la velocidad será nulo, pero su magnitud cambiará en una cantidad que puede ser obtenida considerando la suma de sus cambios cuadráticos. El efecto sobre la excentricidad de este proceso de camino aleatorio resultará, pues, de promediar sobre la órbita el cuadrado de la ecuación anterior. Asumiendo que las perturbaciones se producen con igual probabilidad en todo punto de la órbita obtenemos

$$
\left\langle\left(\frac{\mathrm{d} e}{\mathrm{~d} t}\right)^{2}\right\rangle=\frac{1}{v_{\mathrm{K}}^{2}}\left(\left\langle R^{2}\right\rangle \frac{1}{2}+4\left\langle B^{2}\right\rangle \frac{1}{2}\right) .
$$

Puesto que las perturbaciones pueden tener cualquier dirección $\left\langle R^{2}\right\rangle=\left\langle B^{2}\right\rangle=\left\langle N^{2}\right\rangle$, lo cual implica que

$$
\left\langle R^{2}\right\rangle=\left\langle B^{2}\right\rangle=\frac{1}{3}\left\langle\mathbf{F}^{2}\right\rangle=\frac{1}{3} \frac{\left\langle(\Delta \mathbf{v})^{2}\right\rangle}{(\Delta t)^{2}} \equiv \frac{1}{3} \frac{\Delta v^{2}}{(\Delta t)^{2}}
$$

Reemplazando estas expresiones en la ecuación anterior y aproximando los diferenciales por incrementos finitos obtenemos finalmente la ecuación (5-27).

Nos interesa ahora determinar el cambio de velocidad (y el correspondiente cambio de la excentricidad) debido a los encuentros y colisiones de nuestro planetesimal de masa $m$ con otros planetesimales cercanos al mismo. Comencemos considerando el pasaje de un objeto de masa $m^{\prime}$ en las cercanías del planetesimal $m$ bajo las hipótesis de la aproximación impulsiva. Esto es, la trayectoria de la masa $m^{\prime}$ se supone rectilínea y uniforme no siendo afectada por la presencia del cuerpo que perturba ${ }^{3}$. En tal caso, el cambio de velocidad que experimenta $m$ por el encuentro con $m^{\prime}$ es en la dirección perpendicular a la velocidad relativa $v_{\mathrm{R}}$ entre ambos y su magnitud está dada por

$$
\Delta v=\frac{2 G m^{\prime}}{b v_{\mathrm{R}}}
$$

siendo $b$ el parámetro de impacto.

En efecto, considerando la geometría del problema como se ilustra en la Figura 5.2, las componentes de la fuerza por unidad de masa $\mathbf{F}$ que experimenta el cuerpo de masa $m$ en la dirección de movimiento $F_{/ /}$y transversal a la misma $F_{\perp}$ están dadas por

$$
F_{/ /}=\frac{G m^{\prime}}{r^{2}} \frac{x}{r}=\frac{G m^{\prime} x}{\left(b^{2}+x^{2}\right)^{3 / 2}}=\frac{G m^{\prime} v_{\mathrm{R}} t}{b^{3}}\left[1+\left(\frac{v_{\mathrm{R}} t}{b}\right)^{2}\right]^{-3 / 2},
$$

\footnotetext{
${ }^{3}$ Claramente la aproximación impulsiva sólo es válida en el caso de un encuentro con grandes velocidades relativas, puesto que, en este caso, la órbita de encuentro es una hipérbola de excentricidad muy alta, es decir, prácticamente una recta. Sin embargo, la aproximación impulsiva permite mantener el modelo simple y correcto en primera aproximación.
} 


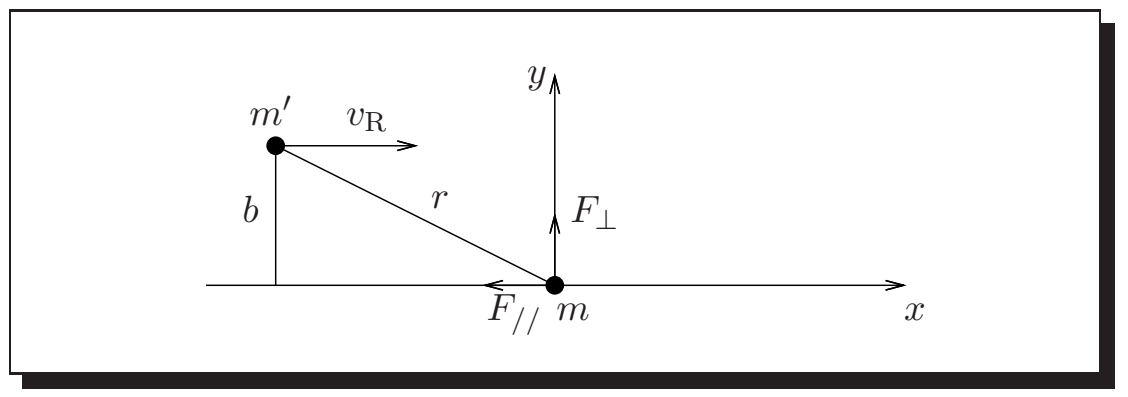

Figura 5.2. Geometría en la aproximación impulsiva de un encuentro. Un planetesimal de masa $m^{\prime}$ se aproxima al planetesimal $m$ sobre una trayectoria rectilínea con velocidad relativa $v_{\mathrm{R}}$ y parámetro de impacto $b$.

$$
F_{\perp}=\frac{G m^{\prime}}{r^{2}} \frac{b}{r}=\frac{G m^{\prime} b}{\left(b^{2}+x^{2}\right)^{3 / 2}}=\frac{G m^{\prime}}{b^{2}}\left[1+\left(\frac{v_{\mathrm{R}} t}{b}\right)^{2}\right]^{-3 / 2},
$$

donde hemos tomado como tiempo inicial al instante en que $m^{\prime}$ pasa por la máxima aproximación. El cambio en la velocidad se obtiene integrando estas ecuaciones sobre el rango temporal $-\infty<t<\infty$. Como $F_{/ /}$es una función impar de $t$ el efecto neto en la dirección del movimiento es nulo, con lo cual el cambio neto en la velocidad está dado solamente por la contribución de $F_{\perp}$ :

$$
\Delta v=\frac{G m^{\prime}}{b v_{\mathrm{R}}} \int_{-\infty}^{\infty}\left(1+\tau^{2}\right)^{-3 / 2} \mathrm{~d} \tau=\frac{2 G m^{\prime}}{b v_{\mathrm{R}}}
$$

como queríamos mostrar.

Consideremos ahora el efecto de sucesivos encuentros con una población de planetesimales descrita, en un dado instante de tiempo, por el número de planetesimales por unidad de volumen, con masas entre $m^{\prime}$ y $m^{\prime}+\mathrm{d} m^{\prime}$ a la distancia $r=a$ sobre el plano del disco:

$$
n\left(m^{\prime}\right) \mathrm{d} m^{\prime}
$$

El número de objetos de masa $m^{\prime}$ que el planetesimal $m$ encuentra con velocidades relativas $v_{\mathrm{R}}$ en un rango del parámetro de impacto $(b, b+\mathrm{d} b)$, y en un intervalo de tiempo $\mathrm{d} t$, es

$$
\mathrm{d} N=n\left(m^{\prime}\right) 2 \pi b \mathrm{~d} b v_{\mathrm{R}} \mathrm{d} t \mathrm{~d} m^{\prime},
$$

suponiendo que la distribución de los objetos es homogénea. Cada encuentro produce una perturbación en la velocidad de $m$ dada por la ecuación (5-28). Nuevamente suponiendo que las direcciones de las velocidades relativas pueden considerarse aleatorias, la magnitud del cambio de velocidad en $m$ está determinada en promedio por su cambio cuadrático medio. Se sigue entonces de las ecuaciones (5-28) y (5-30) e integrando sobre todo el rango del parámetro de impacto y sobre la distribución de masa de la población, que la tasa de cambio de la velocidad por efecto de los encuentros está dada por

$$
\frac{\mathrm{d} v^{2}}{\mathrm{~d} t}=8 \pi G^{2} \ln \Lambda \int_{0}^{m_{\text {máx }}} \frac{m^{\prime 2} n\left(m^{\prime}\right)}{v_{\mathrm{R}}} \mathrm{d} m^{\prime}
$$

donde $\ln \Lambda=\ln \left(b_{\text {máx }} / b_{\text {mín }}\right)$ es el denominado logaritmo coulombiano y $m_{\text {max }}$ es el límite superior en la distribución de masa. 
En efecto, en virtud de la aleatoriedad de los encuentros, la tasa de cambio de la velocidad de $m$ debida a los encuentros con planetesimales de masa $m^{\prime}$ en el rango $(b, b+\mathrm{d} b)$ del parámetro de impacto está dada por

$$
\mathrm{d} v^{2}\left(m^{\prime}, b\right)=\left(\frac{2 G m^{\prime}}{b v_{\mathrm{R}}}\right)^{2} \mathrm{~d} N=8 \pi G^{2} m^{\prime 2} \frac{n\left(m^{\prime}\right)}{v_{\mathrm{R}}} \frac{\mathrm{d} b}{b} \mathrm{~d} m^{\prime} \mathrm{d} t .
$$

Integrando sobre el rango de variación del parámetro de impacto, suponiendo que es independiente de la masa, obtenemos

$$
\mathrm{d} v^{2}\left(m^{\prime}\right)=8 \pi G^{2} m^{\prime 2} \ln \Lambda \frac{n\left(m^{\prime}\right)}{v_{\mathrm{R}}} \mathrm{d} m^{\prime} \mathrm{d} t,
$$

La integración sobre la distribución de masas conduce entonces a la ecuación (5-31).

En virtud de (5-27), la ecuación (5-31) implica que la tasa de variación de la excentricidad de un planetesimal de masa $m$ debido a los encuentros cercanos con el resto de la población está dada por

$$
\frac{\mathrm{d} e^{2}}{\mathrm{~d} t}=\frac{20}{3} \pi G^{2} \ln \Lambda \frac{1}{v_{\mathrm{K}}^{2}} \int_{0}^{m_{\text {máx }}} \frac{m^{\prime 2} n\left(m^{\prime}\right)}{v_{\mathrm{R}}} \mathrm{d} m^{\prime} .
$$

Consideremos ahora los eventos colisionales. Las colisiones macroscópicas son, en general, colisiones inelásticas, esto es, la energía cinética no se conserva sino que es convertida a otras formas, tal como energía interna. En el caso extremo de colisiones perfectamente inelásticas, donde los cuerpos permanecen unidos después del evento, la magnitud del cambio de velocidad que experimenta un cuerpo de masa $m$ producto de la colisión con otro cuerpo de masa $m^{\prime}$ está dada por

$$
\Delta v=\frac{m^{\prime}}{m+m^{\prime}} v_{\mathrm{col}}
$$

donde $v_{\text {col }}$ es la velocidad relativa de colisión.

En efecto, debido a la conservación del impulso lineal, inmediatamente antes y después del choque:

$$
m \mathbf{v}_{i}+m^{\prime} \mathbf{v}_{i}^{\prime}=\left(m+m^{\prime}\right) \mathbf{v}_{f},
$$

de donde se sigue que el cambio en la velocidad de $m$ es

$$
\Delta \mathbf{v}=\mathbf{v}_{f}-\mathbf{v}_{i}=-\frac{m^{\prime}}{m+m^{\prime}}\left(\mathbf{v}_{i}-\mathbf{v}_{i}^{\prime}\right)=-\frac{m^{\prime}}{m+m^{\prime}} \mathbf{v}_{\mathrm{col}} .
$$

y por lo tanto, en magnitud, obtenemos la ecuación planteada.

Si $v_{\mathrm{R}}$ es la velocidad relativa de los cuerpos lejos del encuentro, la velocidad de colisión puede escribirse como

$$
v_{\mathrm{col}}^{2}=v_{\mathrm{R}}^{2}+v_{\mathrm{e}}^{2},
$$

donde $v_{e}$ es la velocidad de escape del punto de contacto

$$
v_{\mathrm{e}}^{2}=2 G \frac{m+m^{\prime}}{R+R^{\prime}}
$$

siendo $R$ y $R^{\prime}$ los radios de los cuerpos de masa $m$ y $m^{\prime}$, respectivamente. 
En efecto, considerando la órbita kepleriana del cuerpo $m^{\prime}$ respecto a $m$, evaluando la integral de la energía $C=\frac{1}{2} v^{2}-\mu / r$, donde $\mu=G\left(m+m^{\prime}\right)$, en el instante del contacto $\left(r=R+R^{\prime}\right)$ y en $r \rightarrow \infty$, resulta

$$
\frac{1}{2} v_{\mathrm{col}}^{2}-G \frac{m+m^{\prime}}{R+R^{\prime}}=\frac{1}{2} v_{\mathrm{R}}^{2}
$$

de donde obtenemos la ecuación (5-34).

Analicemos ahora el efecto de sucesivas colisiones del cuerpo $m$ con la población de objetos descrita según (5-29). Las colisiones ocurrirán cuando la separación entre los centros de los dos objetos en cuestión es menor o igual que la suma de sus radios. Así, el número de objetos de masa $m^{\prime}$ que $m$ encuentra con velocidades relativas $v_{\mathrm{R}}$ para colisión en un intervalo de tiempo dt es

$$
\mathrm{d} N=n\left(m^{\prime}\right) \pi\left(R+R^{\prime}\right)^{2} v_{\mathrm{R}} \mathrm{d} t \mathrm{~d} m^{\prime}
$$

Cada colisión produce una perturbación en la velocidad de $m$ cuya magnitud es dada por la ecuación (5-33). En forma similar a lo anterior, suponiendo que las colisiones ocurren en direcciones aleatorias, la magnitud del cambio de velocidad de $m$ está determinada por la suma cuadrática de las contribuciones de cada colisión. Se sigue entonces de las ecuaciones (5-33), (5-34) y (5-36), integrando sobre toda la distribución de masa, que la tasa de cambio de la velocidad por efecto de las colisiones está dada por

$$
\frac{\mathrm{d} v^{2}}{\mathrm{~d} t}=\pi \int_{0}^{m_{\text {máx }}}\left(R+R^{\prime}\right)^{2}\left[1+\left(\frac{v_{e}}{v_{\mathrm{R}}}\right)^{2}\right] v_{\mathrm{R}}^{3}\left(\frac{m^{\prime}}{m+m^{\prime}}\right)^{2} n\left(m^{\prime}\right) \mathrm{d} m^{\prime} .
$$

En efecto, por la aleatoriedad de las direcciones de las colisiones, la tasa de cambio de la velocidad de $m$ debido a las colisiones con planetesimales de masa $m^{\prime}$ con velocidades relativas $v_{\mathrm{R}}$ está dada por

$$
\begin{aligned}
\mathrm{d} v^{2}\left(m^{\prime}\right) & =\left[\frac{m^{\prime}}{m+m^{\prime}}\left(v_{\mathrm{R}}^{2}+v_{e}^{2}\right)^{1 / 2}\right]^{2} \mathrm{~d} N \\
& =\left(\frac{m^{\prime}}{m+m^{\prime}}\right)^{2} v_{\mathrm{R}}^{3}\left[1+\left(\frac{v_{e}}{v_{\mathrm{R}}}\right)^{2}\right] n\left(m^{\prime}\right) \pi\left(R+R^{\prime}\right)^{2} \mathrm{~d} m^{\prime} \mathrm{d} t .
\end{aligned}
$$

La integración sobre la distribución de masa conduce entonces a la ecuación (5-37).

Utilizando nuevamente la ecuación (5-27), se sigue de (5-37), que la tasa de variación de la excentricidad de un planetesimal de masa $m$ debido a las colisiones con el resto de la población está dada por

$$
\frac{\mathrm{d} e^{2}}{\mathrm{~d} t}=\frac{5}{6} \frac{\pi}{v_{\mathrm{K}}^{2}} \int_{0}^{m_{\text {máx }}}\left(R+R^{\prime}\right)^{2}\left[1+\left(\frac{v_{e}}{v_{\mathrm{R}}}\right)^{2}\right] v_{\mathrm{R}}^{3}\left(\frac{m^{\prime}}{m+m^{\prime}}\right)^{2} n\left(m^{\prime}\right) \mathrm{d} m^{\prime} .
$$

Suponiendo una densidad de masa volumétrica $\rho$ constante para toda la población, y aproximando los planetesimales como esferas,

$$
m=\frac{4}{3} \pi R^{3} \rho,
$$


con lo cual los radios $R, R^{\prime}$ y la velocidad de escape $v_{e}$ en (5-38) pueden expresarse explícitamente en función de la masa:

$$
R=\left(\frac{3}{4 \pi \rho}\right)^{1 / 3} m^{1 / 3}, \quad R^{\prime}=\left(\frac{3}{4 \pi \rho}\right)^{1 / 3} m^{1 / 3},
$$

y

$$
v_{e}^{2}=2 G \frac{m+m^{\prime}}{R+R^{\prime}}=2\left(\frac{4 \pi \rho}{3}\right)^{1 / 3} G \frac{m+m^{\prime}}{m^{1 / 3}+m^{1 / 3}} .
$$

Debido a que, en promedio, los encuentros excitan las excentricidades, en tanto las colisiones enfrían al sistema (Safronov, 1969), la tasa de cambio de la excentricidad de un planetesimal de masa $m$ será

$$
\frac{\mathrm{d} e^{2}}{\mathrm{~d} t}=\left.\frac{\mathrm{d} e^{2}}{\mathrm{~d} t}\right|_{\mathrm{enc}}-\left.\frac{\mathrm{d} e^{2}}{\mathrm{~d} t}\right|_{\mathrm{col}}
$$

donde la tasa debido a los encuentros está dada por (5-32) y la correspondiente a las colisiones por (5-38). Estas ecuaciones constituyen la base de nuestro modelo para describir el efecto de la autointeracción del disco y serán aplicadas, en la siguiente sección, para el caso particular de un disco de partículas de masas iguales.

\subsection{Disco de partículas de masas iguales.}

Supongamos que la población del disco está constituida por planetesimales de masas iguales $m$. En tal caso la función de distribución del número de partículas por unidad de volumen, puede ser descrita como

$$
\boldsymbol{n}\left(m^{\prime}\right) \mathrm{d} m^{\prime}=\mathcal{N} \delta\left(m^{\prime}-m\right) \mathrm{d} m^{\prime}
$$

donde $\mathcal{N}$ es el número de partículas de masa $m$ por unidad de volumen a la distancia $r=a, \mathrm{y} \delta$ es la delta de Dirac.

Reemplazando (5-43) en la ecuación (5-32), la tasa de excitación de la excentricidad por encuentros resulta dada por

$$
\frac{\mathrm{d} e^{2}}{\mathrm{~d} t}=\frac{20}{3} \pi G^{2} \ln \Lambda \frac{\mathcal{N} m^{2}}{v_{\mathrm{K}}^{2} v_{\mathrm{R}}} .
$$

Ahora bien, tanto $\mathcal{N}$ como $v_{\mathrm{R}}$ contienen información sobre el estado dinámico del disco, la cual explicitamos como sigue. En primer lugar, notemos que la densidad superficial $\Sigma$ del disco está relacionada con la distribución de densidad $n$ de la población en $r=a$ y su excentricidad media cuadrática según la ecuación

$$
\mathcal{N}=\frac{\Sigma}{\text { aem }}
$$

En efecto, en un anillo diferencial de ancho da, la masa contenida es

$$
\sum 2 \pi a \mathrm{~d} a .
$$


Siendo $h$ la escala de altura del disco, en el cilindro diferencial de altura $2 h$ la masa contenida es

$$
m \mathcal{N} 2 \pi a \mathrm{~d} a 2 h .
$$

De estas dos ecuaciones se sigue que

$$
\Sigma=2 \mathcal{N} m h .
$$

Por otra parte la escala de altura $h$ del disco puede escribirse (Lissauer y Stewart, 1993), en $r=a$, como

$$
h=a\left\langle i^{2}\right\rangle^{1 / 2}=\frac{a}{2}\left\langle e^{2}\right\rangle^{1 / 2},
$$

donde la inclinación $i$ es expresada en radianes y hemos supuesto la condición de equilibrio dinámico (Stewart y Wetherill, 1988)

$$
\left\langle e^{2}\right\rangle^{1 / 2} /\left\langle i^{2}\right\rangle^{1 / 2}=2 .
$$

De (5-46) y (5-47) se sigue la ecuación (5-45).

Por otra parte, la velocidad relativa promedio $v_{\mathrm{R}}$ entre dos cuerpos está relacionada con la velocidad kepleriana circular local $v_{\mathrm{K}}$ y la excentricidad media cuadrática de la población según

$$
v_{\mathrm{R}}=\sqrt{\frac{5}{2}} v_{\mathrm{K}} e .
$$

En efecto, en una descripción estadística del disco de planetesimales, la distribución de velocidades de los mismos es asumida aleatoria respecto de la velocidad circular kepleriana local $v_{\mathrm{K}}$, estando relacionada con los valores medios cuadráticos de la excentricidad e inclinación (en radianes) de la población de objetos de masa $m$ por (ver Lissauer \& Stewart, 1993)

$$
v=v_{\mathrm{K}}\left(e^{2}+i^{2}\right)^{1 / 2}=\frac{\sqrt{5}}{2} v_{\mathrm{K}} e,
$$

donde hemos asumido nuevamente la condición de equilibrio (5-48). Ahora bien, la velocidad relativa entre dos cuerpos $m$ y $m^{\prime}$ de la población de planetesimales tiene una magnitud dada por $v_{\mathrm{R}}^{2}=\left[\left(\mathbf{v}_{\mathrm{K}}+\mathbf{v}^{\prime}\right)-\left(\mathbf{v}_{\mathrm{K}}+\mathbf{v}\right)\right]^{2}=\left(\mathbf{v}^{\prime}-\mathbf{v}\right)^{2}=v^{2}-2 \mathbf{v} \cdot \mathbf{v}^{\prime}+v^{\prime 2}$. Debido a la aleatoriedad asumida de las velocidades relativas a la velocidad kepleriana, $\left\langle\mathbf{v} \cdot \mathbf{v}^{\prime}\right\rangle=0$, con lo cual, siendo $|\mathbf{v}|=\left|\mathbf{v}^{\prime}\right|=v$,

$$
v_{\mathrm{R}}^{2}=2 v^{2}
$$

De las dos ecuaciones anteriores resulta (5-49).

La sustitución de las ecuaciones (5-45) y (5-49) en (5-44) permiten escribir la evolución de la excentricidad por encuentros como:

$$
e^{2} \frac{\mathrm{d} e^{2}}{\mathrm{~d} t}=\frac{4 \sqrt{10}}{3} \pi \ln \Lambda\left(\frac{m}{m_{0}}\right)^{2} \frac{\sum a^{2}}{m} n .
$$

siendo $n$ el movimiento medio de los planetesimales de masa $m$ a la distancia $r=a$ sobre el plano del disco.

En efecto, sólo es cuestión de álgebra y tener presente que aproximando las órbitas keplerianas por una órbita circular se cumplen las relaciones

$$
v_{K}=n a=\sqrt{\frac{G m_{0}}{a}},
$$


que permiten, entonces, deducir la ecuación planteada.

La ecuación (5-50) puede ser integrada en primera aproximación suponiendo que los parámetros del segundo miembro son constantes sobre un intervalo de tiempo $\tau$. Para una excentricidad inicial nula, la evolución de la excentricidad de la población de objetos al tiempo $\tau$ a la distancia $r=a$ sobre el plano del disco, debida a los encuentros, resulta, entonces, dada aproximadamente por la ecuación

$$
e^{4}=\frac{8 \sqrt{10}}{3} \pi \ln \Lambda\left(\frac{m}{m_{0}}\right)^{2} \frac{\Sigma a^{2}}{m} n \tau .
$$

Consideremos ahora los procesos colisionales. Sustituyendo la función de distribución de la población (5-43) en la ecuación (5-38) y teniendo presente que todos los planetesimales tienen la misma masa y radio, la tasa de cambio de la excentricidad por colisiones resulta dada por:

$$
\frac{\mathrm{d} e^{2}}{\mathrm{~d} t}=\frac{5}{6} \frac{\pi}{v_{\mathrm{K}}^{2}}\left[1+\left(\frac{v_{e}}{v_{\mathrm{R}}}\right)^{2}\right] v_{\mathrm{R}}^{3} \mathcal{N} R^{2} .
$$

En forma similar a lo anterior, utilizamos las ecuaciones (5-45) y (5-49), junto con (5-41) para expresar la evolución de la excentricidad por colisiones como:

$$
\frac{\mathrm{d} e^{2}}{\mathrm{~d} t}=\frac{25}{24} \sqrt{10} \pi\left[e^{2}+\frac{4}{5} \frac{a}{R} \frac{m}{m_{0}}\right] \frac{\sum R^{2}}{m} n .
$$

En efecto, la sustitución directa de tales relaciones, teniendo presente que, para masas iguales, la ecuación (5-41) toma la forma

$$
v_{e}^{2}=\frac{2 G m}{R},
$$

junto con las relaciones, al aproximar las órbitas keplerianas por una órbita circular,

$$
v_{K}=n a=\sqrt{\frac{G m_{0}}{a}}
$$

permiten deducir la ecuación planteada.

La integración de la ecuación (5-53) sobre un intervalo temporal $\tau$, comenzando con excentricidad nula y asumiendo constantes los parámetros involucrados sobre dicho intervalo de tiempo, conduce a que la evolución de la excentricidad de la población de objetos al tiempo $\tau$ a la distancia $r=a$ sobre el plano del disco, debida a las colisiones, resulte dada aproximadamente por la ecuación

$$
e^{2}=\frac{5}{6} \sqrt{10} \pi \frac{a}{R} \frac{m}{m_{0}} \frac{\Sigma R^{2}}{m} n \tau
$$

asumiendo que

$$
\frac{25}{24} \sqrt{10} \pi \frac{\Sigma R^{2}}{m} n \tau \ll 1
$$


En efecto, la separación de variables de la ecuación (5-53) para su integración, suponiendo constantes sobre el intervalo de tiempo $\tau$ los parámetros involucrados y considerando excentricidad inicial nula, conduce a

$$
e^{2}=\frac{4}{5} \frac{a}{R} \frac{m}{m_{0}}\left[\exp \left(\frac{25}{24} \sqrt{10} \pi \frac{\Sigma R^{2}}{m} n \tau\right)-1\right] .
$$

El desarrollo a primer orden en $\tau$ de la función exponencial involucrada en esta ecuación permite obtener la ecuación planteada.

\subsection{Disco de partículas de masas iguales y densi- dad superficial constante.}

Para continuar supondremos que la densidad superficial $\Sigma$ es constante sobre todo el disco, tal como es el caso de las simulaciones presentadas en la introducción. Si tal disco está constituido por $N$ objetos de masa $m$ y se extiende entre $r=a_{\text {mín }}$ y $r=a_{\text {máx }}$, siendo $a_{\text {máx }} \gg a_{\text {mín }}$, con una masa total $M_{\text {disco }}=N m$, podemos escribir:

$$
\Sigma=\frac{N m}{\pi\left(a_{\text {máx }}^{2}-a_{\text {mín }}^{2}\right)} \simeq \frac{N m}{\pi a_{\text {máx }}^{2}}
$$

y se sigue de la ecuación (5-51) que la excitación de la excentricidad por encuentros al tiempo $\tau$ resulta dada por:

$$
e^{2}=\left(\frac{8 \sqrt{10}}{3} \ln \Lambda\right)^{1 / 2} \frac{M_{\text {disco }}}{m_{0}} \frac{a}{a_{\text {máx }}} N^{-1 / 2} n^{1 / 2} \tau^{1 / 2} .
$$

En efecto, sustituyendo esta expresión en (5-51), ésta toma la forma

$$
e^{4}=\frac{8 \sqrt{10}}{3} \ln \Lambda\left(\frac{m}{m_{0}}\right)^{2}\left(\frac{a}{a_{\text {máx }}}\right)^{2} N n \tau,
$$

y puesto que $m=M_{\text {disco }} / N$, obtenemos la ecuación planteada.

De la misma forma, se sigue de la ecuación (5-54), que la evolución temporal de la excentricidad por colisiones al tiempo $\tau$ resulta dada por:

$$
e^{2}=\frac{5}{6} \sqrt{10}\left(\frac{3 M_{\text {disco }}}{4 \pi \rho a_{\text {máx }}^{3}}\right)^{1 / 3} \frac{M_{\text {disco }}}{m_{0}} \frac{a}{a_{\text {máx }}} N^{-1 / 3} n \tau .
$$

En efecto, teniendo en cuenta que la densidad superficial, en este caso, está dada por la ecuación (5-56) se sigue que (5-54) toma la forma

$$
e^{2}=\frac{5}{6} \sqrt{10} \frac{a}{a_{\text {máx }}} \frac{R}{a_{\text {máx }}} \frac{m}{m_{0}} N n \tau .
$$

Haciendo uso de (5-40) junto con la relación $m=M_{\text {disco }} / N$ se sigue la ecuación planteada. 
Las ecuaciones (5-57) y (5-58) modelan la evolución de la excentricidad de la población del disco de densidad constante debido a los encuentros y colisiones, respectivamente, con otros planetesimales, estando la evolución total dictada, según la ecuación (5-42), por

$$
e=\sqrt{e_{\mathrm{enc}}^{2}-e_{\mathrm{col}}^{2}} \text {. }
$$

En particular, la evolución de la excentricidad de los planetesimales, por estos efectos, en la localización resonante correspondiente a una resonancia interna de primer orden $j:(j-1)$ de un cuerpo de masa planetaria $M$ embebido en la población del disco a una distancia $a_{p}$ sobre el plano del disco (con movimiento medio $n_{p}$ ) resulta, a partir incorporar las ecuaciones (5-10) en (5-57) y (5-58), dada por:

$$
\begin{gathered}
e_{\text {enc }}^{2}=\left(\frac{j-1}{j}\right)^{1 / 6}\left(\frac{8 \sqrt{10}}{3} \ln \Lambda\right)^{1 / 2} \frac{M_{\text {disco }}}{m_{0}} \frac{a_{p}}{a_{\text {máx }}} N^{-1 / 2} n_{p}^{1 / 2} \tau^{1 / 2}, \\
e_{\text {col }}^{2}=\frac{5}{6} \sqrt{10}\left(\frac{j-1}{j}\right)^{-1 / 3}\left(\frac{3 M_{\text {disco }}}{4 \pi \rho a_{\text {máx }}^{3}}\right)^{1 / 3} \frac{M_{\text {disco }}}{m_{0}} \frac{a_{p}}{a_{\text {máx }}} N^{-1 / 3} n_{p} \tau .
\end{gathered}
$$

Estas ecuaciones nos permiten analizar los resultados de las simulaciones presentadas en la introducción considerando la evolución de la excentricidad por los efectos de autointeracción en función del número de partículas $N$ utilizado para modelar el disco de masa total $M_{\text {disco }}$ fija. Específicamente, los parámetros del disco de nuestra simulación son:

$$
M_{\text {disco }}=200 M_{\oplus}, \quad a_{\text {máx }}=12.5 \mathrm{UA}, \quad \rho=2 \mathrm{~g} \mathrm{~cm}^{-3}, \quad m_{0}=1 M_{\odot},
$$

y adoptamos un valor típico (Armitage, 2010),

$$
\ln \Lambda \simeq 10 \text {. }
$$

Para el planeta:

$$
M=10 M_{\oplus}, \quad a_{p}=5 \mathrm{UA} .
$$

Además, dado que $n_{p}=\left(G m_{0} / a_{p}^{3}\right)^{1 / 2}$, y utilizando, como en la simulación, la unidad de distancia en UA, el tiempo en años y las masas en masas solares, resulta $G=4 \pi^{2}$, y por lo tanto

$$
n_{p}=\frac{2 \pi}{a_{p}^{3 / 2}}=0.562
$$

Las localizaciones resonantes de interés para las simulaciones presentadas en la introducción corresponden a la resonancias $2: 1(j=2)$ y $3: 2(j=3)$. Es fácil ver que para tales resonancias la contribución de la razón de enteros en las ecuaciones (5-60) y (5-61) es próxima a la unidad y al tomarla como tal eliminamos la dependencia de las mismas con la resonancia particular considerada. Los valores críticos de captura correspondientes, calculados a partir de las ecuaciones (5-19) y (5-20) son:

$$
e_{\text {crit }}^{(2: 1)}=0.0479, \quad e_{\text {crit }}^{(3: 2)}=0.0384 .
$$

La Figura 5.3 muestra el cambio cuadrático de la excentricidad en las localizaciones resonantes por encuentros y por colisiones en función del número de partículas utilizado para modelar el disco, tal como resulta de las ecuaciones (5-60) y (5-61). Es claro 

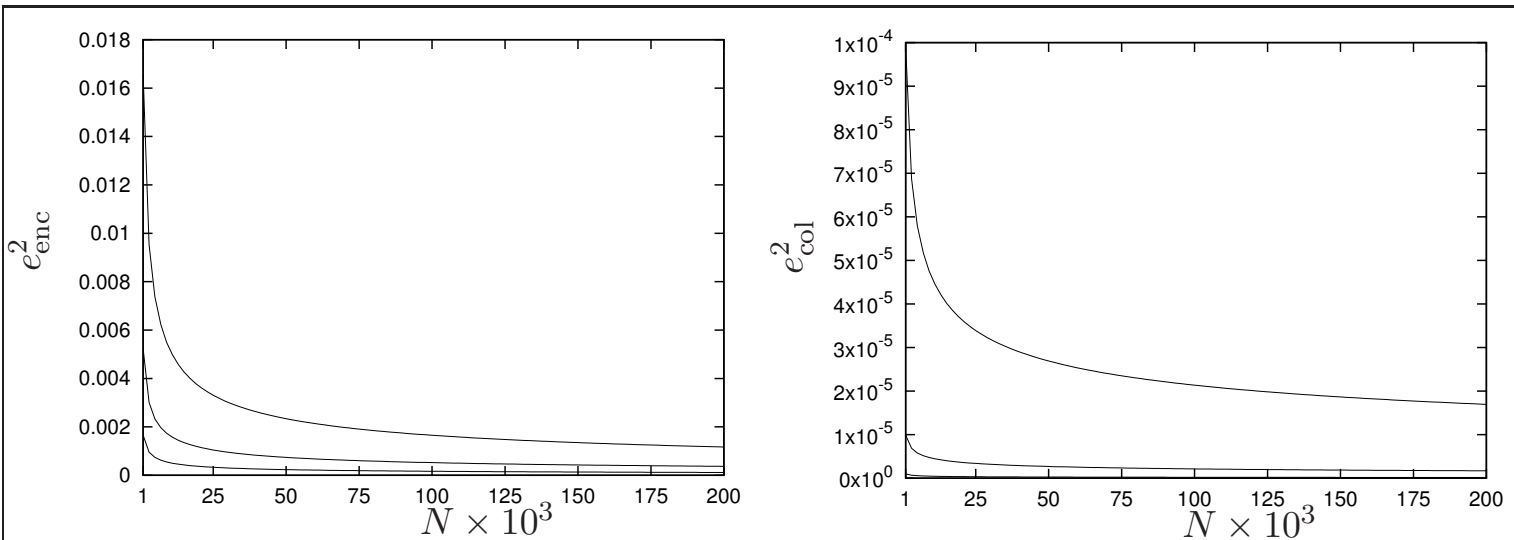

Figura 5.3. Evolución de $e^{2}$ por encuentros y colisiones, respectivamente, en las localizaciones resonantes 2:1 y 3:2 para los intervalos temporales (de abajo hacia arriba) $\tau=10^{3}, 10^{4}$ y $10^{5}$ años en función del número de partículas $N$ para un disco de $M_{\text {disco }}=200 M_{\oplus}$.

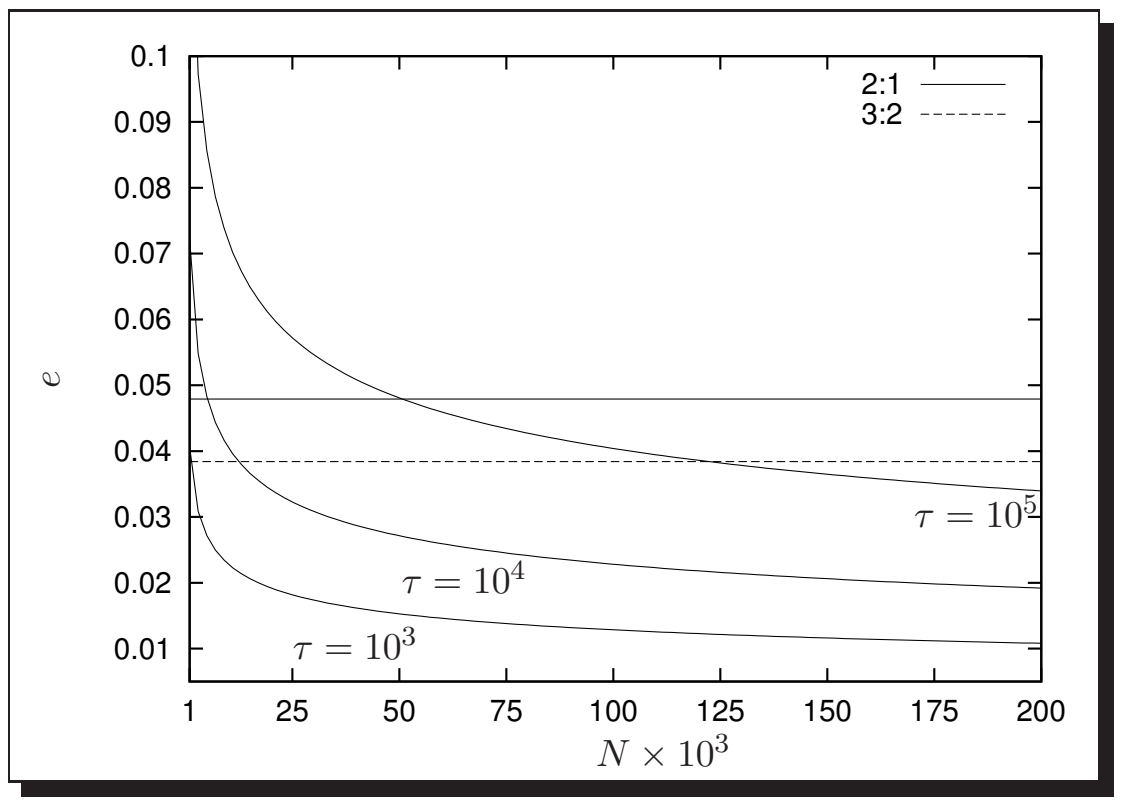

Figura 5.4. Evolución de la excentricidad por autointeracción (encuentros y colisiones) en las localizaciones resonantes 2:1 y 3:2 para diversos intervalos temporales $\tau$ (en años) en función del número de partículas $N$ para un disco de $M_{\text {disco }}=200 M_{\oplus}$. La línea recta sólida corresponde al valor crítico de la excentricidad para la captura en la resonancia interna 2:1, en tanto que la línea recta punteada al correspondiente valor para la resonancia interna $3: 2$. 
de los gráficos que la contribución de las colisiones al proceso es mínima frente a los encuentros gravitacionales. Finalmente, la Figura 5.4 da cuenta de la evolución total de la excentricidad en función del número de partículas $N$ y es claro que, al tiempo $\tau=10^{5}$ años, se requiere un disco con $N>50000$ partículas para reproducir el escenario de captura en resonancia 2:1 en una simulación que contemple la autointeracción y un disco con $N>125000$ para el caso de la resonancia 3:2. Las ecuaciones planteadas explican, así, porque en una simulación con un disco autointeractuante de $N=10000$ partículas tales resonancias resultan difusas. Por otra parte es claro que simulaciones con discos autointeractuantes compuestos de un número tan alto de partículas como los requeridos resultan inviables, al momento, debido al gran costo de tiempo computacional involucrado. 


\section{Capítulo 6}

\section{Conclusiones y perspectivas}

En esta tesis presentamos algunos aspectos relevantes a las simulaciones numéricas de la interacción planeta-disco para uno o más planetas embebidos en un disco de planetesimales remanente del proceso de formación planetaria, libre de gas.

Para explorar tales aspectos ampliamos la capacidad de un código numérico, desarollado originalmente en la tesis de licenciatura del autor, de manera que resulte adecuado al escenario bajo estudio. Dicho código tiene las siguientes características: $(i)$ es un integrador simpléctico y por lo tanto preserva la evolución dinámica del sistema con variaciones en el cómputo de la energía acotadas, sin introducir componentes seculares, aun durante encuentros entre los cuerpos; (ii) aprovecha el hecho de que, en ausencia de encuentros, los cuerpos se mueven principalmente bajo un órbita kepleriana en torno al cuerpo central, la cual puede entonces computarse analíticamente vía el problema de dos cuerpos; (iii) los encuentros próximos entre cuerpos son detectados y resueltos, sin destruir la naturaleza simpléctica del integrador, como un problema modificado de tres cuerpos mediante un integrador numérico clásico; (iv) el cálculo de las interacciones gravitatorias mutuas procede por el esquema de árbol octal, con la consiguiente disminución del tiempo de cómputo respecto a una evaluación directa de tales interacciones o, en forma equivalente, la posibilidad de considerar un mayor número de cuerpos; $(v)$ permite diferenciar una población de cuerpos planetarios de las partículas que modelan el disco de planetesimales de manera tal que tales partículas interactúan gravitacionalmente con los planetas pero no entre sí.

El código ampliado ha reproducido satisfactoriamente varios escenarios de interacción planeta-disco presentes en la literatura, a saber, $(i)$ la migración hacia el interior de un cuerpo planetario sumergido en el disco de planetesimales vía fricción dinámica (Cionco y Brunini, 2002); (ii) la migración en fuga de Neptuno en un sistema solar exterior inicialmente más compacto (Gomes y otros, 2004); (iii) el escenario para el bombardeo intenso tardío dentro del marco del modelo de Niza (Gomes y otros, 2005).

Gracias a la incorporación del esquema de árbol octal en la evaluación de las interacciones gravitatorias mutuas, fue posible abordar, en forma sistemática uno de los puntos relevantes de las simulaciones, a saber, el problema de la sensibilidad de las mismas respecto al número de partículas utilizado para modelar el disco de planetesimales. Utilizando el modelo estadístico de "partícula en una caja" (Stewart y Wetherill, 1988) pudimos fundamentar que cuando la "granularidad" de la simulación, definida por la 
razón de la masa $M$ del planeta a la masa individual $m$ de cada planetesimal, es menor que cierto valor crítico el efecto de la fricción dinámica operando en la interacción planeta-disco es pobremente modelada, siendo superada por los efectos estocásticos de los encuentros cercanos con partículas de masas no realistas.

Finalmente abordamos un segundo punto de interés: la influencia de la autointeracción del disco, típicamente despreciada en las simulaciones numéricas, en las poblaciones originadas por la captura en resonancias de movimientos medios conforme el planeta migra. A través de un modelo simplificado que da cuenta de la excitación de las partículas del disco por sus interacciones mutuas exploramos cuál debería ser el número de partículas a considerar en una simulación que podría reproducir adecuadamente tales poblaciones si la autointeracción es incluida. Tal análisis nos permitió concluir que dicho número es prohibitivamente alto en tiempo computacional, pero permite explicar por qué aun una simulación con granularidad correcta para modelar la migración planetaria no necesariamente es suficiente para describir las poblaciones resonantes si la autointeracción es considerada.

Como todo programa informático, el código desarrollado es susceptible de mejoras. Uno de los puntos de interés a considerar a futuro es la incorporación de la capacidad de concurrencia y paralelismo. El autor de esta tesis se encuentra actualmente estudiando estos recursos computacionales. En una primera etapa una mejora significativa consiste en la posibilidad de utilizar simultáneamente los cores disponibles en los multiprocesadores actuales a través de la incorporación de directivas OpenMP sobre las estructuras de control iterativas (sentencias DO ... END DO de Fortran) de manera que las mismas sea ejecutada concurrentemente a través de todos los cores. En una segunda etapa, se puede considerar la paralelización del código para ser ejecutado ya sea en un cluster de cómputo o bien en hardware específico como GPUs o coprocesadores Intel Xeon-Phi. Sin embargo, ésto requiere una adaptación mayor del código ya que se requiere de un adecuado estudio de la forma óptima de particionar el computo de manera de minimizar todo tiempo ocioso del hardware. A este respecto, en las simulaciones de nuestro escenario, los encuentros entre cuerpos imponen una barrera natural en el desarrollo del cómputo, por cuanto la resolución de un encuentro requiere, usualmente, en el código híbrido, de muchos pasos de integración para alcanzar el tiempo al cual se quiere evaluar el sistema. Así todo otro cálculo, que dependa de la posición e impulsos de los cuerpos en encuentro, estará detenido hasta la espera de que el mismo sea resuelto. 


\section{Apéndice A}

\section{Elementos de la formulación hamiltoniana de la mecánica clásica}

Ecuaciones de movimiento de Hamilton. Las ecuaciones de movimiento de un sistema físico de, digamos, $n$ grados de libertad, pueden describirse por un conjunto de $2 n$ ecuaciones diferenciales de primer orden para un conjunto de $2 n$ variables independientes: a saber $n$ coordenadas generalizadas $q_{i}$ y sus respectivos $n$ impulsos canónicos conjugados $p_{i}$, a través de una función de las coordenadas e impulsos llamada el hamiltoniano del sistema,

$$
H=H(\mathbf{q}, \mathbf{p}) \text {. }
$$

Tal conjunto de ecuaciones diferenciales son las ecuaciones canónicas de Hamilton:

$$
\begin{aligned}
& \dot{q}_{i}=\frac{\partial H}{\partial p_{i}} \\
& \dot{p}_{i}=-\frac{\partial H}{\partial q_{i}} .
\end{aligned}
$$

El conjunto de $2 n$ variables independientes que constituyen los $q_{i}$ y $p_{i}$ nos permite definir un espacio cartesiano $2 n$-dimensional, denominado espacio de las fases, asociando a cada coordenada $q_{i}$ e impulso $p_{i}$ un eje cartesiano. Cuando, en un instante dado, se conocen las posiciones e impulsos del sistema, éste queda representado por un punto en el espacio de las fases, y su evolución futura, dada por las ecuaciones de movimiento, traza una curva en el espacio de las fases, la cual representa la evolución del sistema.

Las ecuaciones de movimiento de Hamilton pueden ser escritas en un esquema de nomenclatura compacto que nos será de utilidad en lo que sigue. Para un sistema de $n$ grados de libertad, construyamos una matriz columna $\boldsymbol{\eta}$ de $2 n$ elementos:

$$
\eta_{i}=q_{i}, \quad \eta_{i+n}=p_{i} ; \quad i=1, \ldots, n
$$

y una matriz columna $\frac{\partial H}{\partial \eta}$ de $2 n$ elementos:

$$
\left(\frac{\partial H}{\partial \boldsymbol{\eta}}\right)_{i}=\frac{\partial H}{\partial q_{i}}, \quad\left(\frac{\partial H}{\partial \boldsymbol{\eta}}\right)_{i+n}=\frac{\partial H}{\partial p_{i}} ; \quad i=1, \ldots, n
$$


Además sea J la matriz cuadrada antisimétrica de $2 n \times 2 n$ construida a partir de matrices nulas y unidad $n \times n$ como:

$$
\mathbf{J}=\left(\begin{array}{cc}
0 & 1 \\
-1 & 0
\end{array}\right)
$$

Entonces las ecuaciones de movimiento de Hamilton pueden escribirse como

$$
\dot{\boldsymbol{\eta}}=\mathbf{J} \frac{\partial H}{\partial \boldsymbol{\eta}}
$$

Esta representación de las ecuaciones de Hamilton es conocida como forma matricial o simpléctica. Es fácil verificar que la matriz $\mathbf{J}$ satisface las siguientes propiedades

$$
\begin{aligned}
\mathbf{J}^{2} & =-\mathbf{I}, \\
\mathbf{J}^{t} \mathbf{J} & =\mathbf{I} \quad(\text { ortogonalidad), } \\
\mathbf{J}^{t} & =-\mathbf{J}=\mathbf{J}^{-1}, \\
\operatorname{det}(\mathbf{J}) & =+1
\end{aligned}
$$

Transformaciones canónicas. Para nuestros propósitos resulta suficiente tratar con sistemas conservativos en donde el hamiltoniano no depende explícitamente del tiempo y puede identificarse con la energía total del sistema (y por lo tanto se conserva). En tal sistema consideremos una transformación simultánea de las coordenadas e impulsos, $q_{i}$ y $p_{i}$, a un nuevo sistema $Q_{i}$ y $P_{i}$ a partir de ciertas ecuaciones (invertibles)

$$
\begin{aligned}
Q_{i} & =Q_{i}(\mathbf{q}, \mathbf{p}), \\
P_{i} & =P_{i}(\mathbf{q}, \mathbf{p}),
\end{aligned}
$$

o, en forma matricial, definiendo las matrices columna $\boldsymbol{\eta}$ y $\boldsymbol{\zeta}$ de $2 n$ elementos $q_{i}, p_{i}$ y $Q_{i}, P_{i}$, respectivamente

$$
\zeta=\zeta(\eta)
$$

Estas ecuaciones definen una transformación del espacio de las fases del sistema y se dice que es una transformación canónica si para todo hamiltoniano $H(q, p)$ se preserva la estructura de las ecuaciones de movimiento. Esto es si existe otra función $K(Q, P)$ tal que

$$
\dot{Q}_{i}=\frac{\partial K}{\partial P_{i}}, \quad \dot{P}_{i}=-\frac{\partial K}{\partial Q_{i}},
$$

o, en notación simpléctica

$$
\dot{\zeta}=\mathbf{J} \frac{\partial K}{\partial \zeta}
$$

Debido a que, bajo nuestras suposiciones, en las ecuaciones de transformación (A-8) el tiempo no aparece explícitamente, las transformaciones canónicas que consideraremos son realmente transformaciones canónicas restringidas. La caracterización de las transformaciones canónicas puede ser dada bajo tres aspectos (a saber, a través de funciones generatrices, condición simpléctica, o corchetes de Poisson) como discutiremos en las siguientes secciones. Tales métodos, desde luego, están relacionados, y cualquiera de ellos 
es válido para el tratamiento de las transformaciones canónicas. En particular, puede utilizarse indistintamente el formalismo generador o el simpléctico, para mostrar que las transformaciones canónicas tienen las cuatro propiedades siguientes, características de la estructura de grupo:

I. La transformación identidad es canónica.

II. La inversa de una transformación canónica es también canónica.

III. La aplicación sucesiva de dos transformaciones canónicas (operación "producto") es una transformación canónica.

IV. La operación producto es asociativa.

Formalismo generador de las transformaciones canónicas. Utilizando el principio variacional que ha de cumplir todo sistema de coordenadas canónicas, puede mostrarse $^{1}$ que la transformación entre los dos sistemas de coordenadas canónicas (q, p) y $(\mathbf{Q}, \mathbf{P})$ satisface la relación

$$
\sum_{i=1}^{n} p_{i} \dot{q}_{i}-H(\mathbf{q}, \mathbf{p})=\sum_{i=1}^{n} P_{i} \dot{Q}_{i}-K(\mathbf{Q}, \mathbf{P})+\frac{\mathrm{d}}{\mathrm{d} t} F(\mathbf{q}, \mathbf{p}, \mathbf{Q}, \mathbf{P})
$$

salvo un factor de escala que es tomado igual a la unidad. Aquí, $F$ es una función cualquiera de las coordenadas del espacio de las fases con derivadas segundas continuas. Mediante las ecuaciones de transformación (A-8) y sus inversas, $F$ puede expresarse en parte en función de variables originales y en parte en términos de las variables nuevas. De hecho, $F$ resulta útil para especificar la forma exacta de la transformación canónica sólo cuando la mitad de las variables pertenece al sistema original y el resto al nuevo. De esta forma, $F$ constituye un puente entre los dos sistemas canónicos y se denomina así función generatriz de la transformación canónica. Para ilustrar cómo una función generatriz determina las ecuaciones de transformación, supongamos que $F$ es especificada como

$$
F=\sum_{i}^{n} q_{i} p_{i}+F_{3}(\mathbf{Q}, \mathbf{p}),
$$

donde $F_{3}$ es una cierta función de los impulsos originales $p_{i}$ y las nuevas coordenadas $Q_{i}$. La ecuación (A-12) toma la forma

$$
\begin{aligned}
-\sum_{i=1}^{n} q_{i} \dot{p}_{i}-H(\mathbf{q}, \mathbf{p}) & =\sum_{i=1}^{n} P_{i} \dot{Q}_{i}-K(\mathbf{Q}, \mathbf{P})+\frac{\mathrm{d}}{\mathrm{d} t} F_{3}(\mathbf{Q}, \mathbf{p}) \\
& =\sum_{i=1}^{n} P_{i} \dot{Q}_{i}-K(\mathbf{Q}, \mathbf{P})+\sum_{i=1}^{n} \frac{\partial F_{3}}{\partial Q_{i}} \dot{Q}_{i}+\sum_{i=1}^{n} \frac{\partial F_{3}}{\partial p_{i}} \dot{p}_{i}
\end{aligned}
$$

Como las coordenadas nuevas $Q_{i}$ y los impulsos originales $p_{i}$ son independientes por separado, esta ecuación se cumplirá idénticamente tan sólo si se anulan los coeficientes de los $\dot{p}_{i}$ y los $\dot{Q}_{i}$, con lo cual

$$
\begin{aligned}
q_{i} & =-\frac{\partial F_{3}}{\partial p_{i}} \\
P_{i} & =-\frac{\partial F_{3}}{\partial Q_{i}}
\end{aligned}
$$

\footnotetext{
${ }^{1}$ Ver, por ejemplo, Goldstein (1990), capítulo 9, sección 1.
} 
en tanto que

$$
K(Q, P)=H(q, p) .
$$

Las ecuaciones (A-14a) constituyen $n$ relaciones que definen los $q_{i}$ en términos de $p_{j}$ y $Q_{j}$, cuya inversión permite obtener las $n Q_{i}$ en función de $q_{j}$ y $p_{j}$, esto es, la primera mitad de las ecuaciones de transformación (A-8). Ahora, sustituyendo estas relaciones en las ecuaciones (A-14b), obtenemos los $n P_{i}$ en función de $q_{j}$ y $p_{j}$, es decir, la segunda mitad de las ecuaciones de transformación (A-8). Finalmente, la ecuación (A-15) proporciona la relación existente entre el nuevo hamiltoniano $K$ y el original $H$, al expresar los $q$ y $p$ de $H$ mediante las inversas de las ecuaciones de transformación (A-8). Incidentalmente (A-15) muestra que para una transformación canónica restringida el hamiltoniano original expresado en las nuevas variables sirve de hamiltoniano nuevo.

Formalismo simpléctico de las transformaciones canónicas. El segundo formalismo para el tratamiento de las transformaciones canónicas resulta de la formulación simpléctica de las ecuaciones de Hamilton. Si $\boldsymbol{\eta}$ es la matriz columna con los $2 n$ elementos $q_{i}, p_{i}$, recordemos que, según la ecuación (A-6), las ecuaciones de Hamilton pueden escribirse como

$$
\dot{\boldsymbol{\eta}}=\mathbf{J} \frac{\partial H}{\partial \boldsymbol{\eta}}
$$

donde $\mathbf{J}$ es la matriz definida en la ecuación (A-5). Por supuesto, en el nuevo sistema de coordenadas, $Q_{i}$ y $P_{i}$ definen una matriz columna $\boldsymbol{\zeta}$ de $2 n$ elementos, y la transformación puede ser escrita según la ecuación (A-9)

$$
\zeta=\zeta(\boldsymbol{\eta})
$$

Entonces la transformación es canónica si y solo si cumple la llamada condición simpléctica

$$
\mathbf{M} \mathbf{J M} \mathbf{M}^{t}=\mathbf{J}
$$

donde $\mathbf{M}=\frac{\partial \zeta}{\partial \eta}$ es la matriz jacobiana $2 n \times 2 n$ de la transformación (A-9), de elementos

$$
M_{i j}=\frac{\partial \zeta_{i}}{\partial \eta_{j}}
$$

Veamos que, efectivamente, la condición simpléctica es una condición suficiente para que la transformación sea canónica. La derivada respecto al tiempo de un elemento cualquiera de $\boldsymbol{\zeta}$ es

$$
\dot{\zeta}_{i}=\sum_{j=1}^{2 n} \frac{\partial \zeta_{i}}{\partial \eta_{j}} \dot{\eta}_{j} ; \quad i=1, \ldots, 2 n
$$

o, en notación matricial

$$
\dot{\zeta}=\mathrm{M} \dot{\eta}
$$

Con las ecuaciones de movimiento para $\boldsymbol{\eta}$, la ecuación (A-18) puede ser escrita como

$$
\dot{\boldsymbol{\zeta}}=\mathbf{M J} \frac{\partial H}{\partial \boldsymbol{\eta}}
$$


A partir de la transformación inversa podemos considerar $H$ como una función de $\boldsymbol{\zeta}$, de manera que su derivada respecto a $\eta_{i}$ es, de acuerdo a la regla de la cadena

$$
\frac{\partial H}{\partial \eta_{i}}=\sum_{j=1}^{2 n} \frac{\partial H}{\partial \zeta_{j}} \frac{\partial \zeta_{j}}{\partial \eta_{i}},
$$

o, en notación matricial

$$
\frac{\partial H}{\partial \boldsymbol{\eta}}=\mathbf{M}^{t} \frac{\partial H}{\partial \boldsymbol{\zeta}} .
$$

Reemplazando la ecuación (A-20) en la ecuación (A-19), obtenemos las ecuaciones de movimiento correspondientes a las variables $\boldsymbol{\zeta}$ obtenidas por la transformación independiente del tiempo (A-9) de las variables canónicas $\boldsymbol{\eta}$

$$
\dot{\boldsymbol{\zeta}}=\mathbf{M} \mathbf{J M}^{t} \frac{\partial H}{\partial \zeta}
$$

Sabemos ya, de la sección anterior, que en el caso de una transformación canónica restringida, el hamiltoniano original expresado en función de las nuevas variables sirve de hamiltoniano nuevo. De esta forma

$$
\dot{\zeta}=\mathbf{J} \frac{\partial H}{\partial \zeta}
$$

Por lo tanto, la transformación (A-9) será una transformación canónica, si su matriz jacobiana $\mathbf{M}$ cumple con la condición

$$
\mathbf{M J M}^{t}=\mathbf{J}
$$

tal como queríamos probar. Recíprocamente, invirtiendo los pasos de la demostración, es claro que toda transformación canónica restringida satisface la ecuación (A-16). Indiquemos, sin demostración, que aun en el caso de una transformación que contenga al tiempo en forma explícita, la condición simpléctica es una condición necesaria y suficiente para que la transformación sea canónica² ${ }^{2}$

La condición simpléctica puede ser escrita en una forma alternativa como sigue. Multiplicando a la derecha la ecuación $(\mathrm{A}-16)$ por la matriz inversa de $\mathbf{M}^{t}$ tenemos que

$$
\mathbf{M J}=\mathbf{J}\left(\mathbf{M}^{t}\right)^{-1} .
$$

Multiplicando ahora esta ecuación por la izquierda por $\mathbf{J}$ y por la derecha por $-\mathbf{J}$, obtenemos, en virtud de $(\mathrm{A}-7 \mathrm{a})$

$$
\mathbf{J M}=\left(\mathbf{M}^{t}\right)^{-1} \mathbf{J}
$$

o sea

$$
\mathbf{M}^{t} \mathbf{J} \mathbf{M}=\mathbf{J} .
$$

\footnotetext{
${ }^{2}$ Ver, por ejemplo, Goldstein (1990), Capítulo 9, Sección 3.
} 
Corchetes de Poisson. Si $u$ y $v$ son dos funciones escalares de las variables canónicas $(q, p)$, se define el corchete de Poisson de $u$ y $v$ como

$$
[u, v]_{\mathbf{q}, \mathbf{p}}=\sum_{i=1}^{n}\left(\frac{\partial u}{\partial q_{i}} \frac{\partial v}{\partial p_{i}}-\frac{\partial u}{\partial p_{i}} \frac{\partial v}{\partial q_{i}}\right)=\left(\frac{\partial u}{\partial \boldsymbol{\eta}}\right)^{t} \mathbf{J} \frac{\partial v}{\partial \boldsymbol{\eta}} .
$$

Escogiendo en particular como funciones $u$ y $v$ a las propias variables canónicas (q, p) es fácil verificar que los corchetes de Poisson respectivos, denominados corchetes de Poisson fundamentales, tienen los valores

$$
\begin{aligned}
& {\left[q_{j}, q_{k}\right]_{\mathbf{q}, \mathbf{p}}=\left[p_{j}, p_{k}\right]_{\mathbf{q}, \mathbf{p}}=0,} \\
& {\left[q_{j}, p_{k}\right]_{\mathbf{q}, \mathbf{p}}=-\left[p_{j}, q_{k}\right]_{\mathbf{q}, \mathbf{p}}=\delta_{j k}}
\end{aligned}
$$

Definiendo un corchete de Poisson matriz cuadrada, $[\boldsymbol{\eta}, \boldsymbol{\eta}]$ de manera tal que

$$
([\boldsymbol{\eta}, \boldsymbol{\eta}])_{l m}=\left[\eta_{l}, \eta_{m}\right]
$$

las ecuaciones $(\mathrm{A}-24)$ pueden resumirse como

$$
[\boldsymbol{\eta}, \boldsymbol{\eta}]_{\eta}=\mathbf{J}
$$

Tomando ahora como funciones $u$ y $v$ a las variables transformadas $(\mathbf{Q}, \mathbf{P})$ por las ecuaciones de transformación (A-9) obtenemos el corchete de Poisson matricial:

$$
[\boldsymbol{\zeta}, \boldsymbol{\zeta}]_{\eta}=\left(\frac{\partial \boldsymbol{\zeta}}{\partial \boldsymbol{\eta}}\right)^{t} \mathbf{J} \frac{\partial \boldsymbol{\zeta}}{\partial \boldsymbol{\eta}}=\mathbf{M}^{t} \mathbf{J} \mathbf{M}
$$

donde $\mathbf{M}=\frac{\partial \zeta}{\partial \eta}$ es la matriz jacobiana de la transformación. Entonces si la transformación (A-9) es canónica se cumple la condición simpléctica (A-22) y por lo tanto

$$
[\zeta, \zeta]_{\eta}=\mathbf{J}
$$

Recíprocamente, si es válida la ecuación (A-27), la transformación es canónica. De este modo, la ecuación (A-27) es una condición necesaria y suficiente para que una transformación del espacio de las fases sea canónica.

Si $\boldsymbol{\zeta}$ constituye realmente un nuevo conjunto de variables canónicas, tenemos según la ecuación (A-25) que

$$
[\zeta, \zeta]_{\zeta}=\mathbf{J}
$$

Las ecuaciones $(\mathrm{A}-27)$ y $(\mathrm{A}-28)$ muestran que los corchetes de Poisson fundamentales de las variables $\boldsymbol{\zeta}$ tendrán el mismo valor al calcularlos respecto de cualquier sistema de coordenadas canónicas. Esto es, los corchetes de Poisson fundamentales son invariantes ante las transformaciones canónicas. No es difícil mostrar ${ }^{3}$ que, de hecho, todos los corchetes de Poisson son invariantes ante las transformaciones canónicas. En virtud de este importante resultado, es posible suprimir en la notación anterior el subíndice que indica respecto a qué sistema de coordenadas canónicas se calcula tal corchete.

\footnotetext{
${ }^{3}$ Ver, por ejemplo, Goldstein (1990), Capítulo 9, Sección 4.
} 
Invarianza del elemento de volumen en el espacio de las fases. Además de los corchetes de Poisson, otro invariante canónico lo constituye el elemento de volumen del espacio de las fases. En una transformación $\boldsymbol{\zeta}=\boldsymbol{\zeta}(\boldsymbol{\eta})$ del espacio de las fases el elemento de volumen

$$
\mathrm{d} \boldsymbol{\eta}=\mathrm{d} q_{1} \ldots \mathrm{d} q_{n} \mathrm{~d} p_{1} \ldots \mathrm{d} p_{n},
$$

se transforma en un nuevo elemento de volumen

$$
\mathrm{d} \boldsymbol{\zeta}=\mathrm{d} Q_{1} \ldots \mathrm{d} Q_{n} \mathrm{~d} P_{1} \ldots \mathrm{d} P_{n}
$$

Ahora bien, en toda transformación, los tamaños de los dos elementos de volumen están relacionados con el valor absoluto del determinante de la matriz jacobiana de la transformación según

$$
\mathrm{d} \boldsymbol{\zeta}=|\operatorname{det}(\mathbf{M})| \mathrm{d} \boldsymbol{\eta}
$$

Si la transformación es canónica, de la condición simpléctica (A-16)

$$
\operatorname{det}(\mathbf{M})^{2} \operatorname{det}(\mathbf{J})=\operatorname{det}(\mathbf{J})
$$

con lo cual, puesto que la ecuación $(\mathrm{A}-7 \mathrm{~d}) \operatorname{nos}$ asegurá que $\operatorname{det}(\mathbf{J}) \neq 0$,

$$
|\operatorname{det}(\mathbf{M})|=1
$$

La ecuación (A-29) muestra entonces, según (A-30), que el elemento de volumen del espacio de las fases es, efectivamente, invariante ante transformaciones canónicas. Como corolario, tenemos que el volumen de una región cualquiera del espacio de las fases

$$
\int \cdots \int \mathrm{d} \boldsymbol{\eta}
$$

es también invariante frente a las transformaciones canónicas.

Ecuaciones de movimiento y evolución temporal. Retomemos las ecuaciones canónicas de Hamilton (A-2),

$$
\dot{q}_{i}=\frac{\partial H}{\partial p_{i}}, \quad \dot{p}_{i}=-\frac{\partial H}{\partial q_{i}} .
$$

La resolución de estas ecuaciones diferenciales determinan los valores de $\mathbf{q}$ y $\mathbf{p}$ en un instante de tiempo cualquiera a partir de sus valores en un instante inicial prefijado $t_{0}=0$. Ahora bien, es claro que debe existir una transformación canónica que lleve los valores iniciales constantes de las coordenadas e impulsos a sus respectivos valores en un instante de tiempo posterior $t$ dado:

$$
(\mathbf{q}(0), \mathbf{p}(0)) \rightarrow(\mathbf{q}(t), \mathbf{p}(t)) .
$$

Obtener dicha transformación es equivalente a resolver las ecuaciones de movimiento del problema. Nótese que en esta interpretación del problema, la transformación canónica involucrada juega un rol activo, en el sentido de que ella relaciona las coordenadas de un punto del espacio de las fases con las de otro punto del mismo espacio de las 


\section{A. Elementos de la formulación hamiltoniana de la mecánica clásica}

fases. Esta interpretación es ciertamente diferente (pero fructífera para nuestros propósitos) a la manera en que definimos a las transformaciones canónicas como cambios de coordenadas de espacios de fase. Ahora, en la interpretación activa, la transformación canónica "mueve" el punto figurativo del sistema de una posición de coordenadas $(\mathbf{q}(0), \mathbf{p}(0))$ a otro punto $(\mathbf{Q}, \mathbf{P})=(\mathbf{q}(t), \mathbf{p}(t))$ del espacio de las fases.

Consideremos la evolución en el espacio de las fases, de una cierta función de las variables canónicas $u=u(q, p)$. Usando las ecuaciones (A-2), su derivada total respecto al tiempo puede ser expresada como

$$
\begin{aligned}
\frac{\mathrm{d} u}{\mathrm{~d} t} & =\sum_{i=1}^{n}\left(\frac{\partial u}{\partial q_{i}} \dot{q}_{i}+\frac{\partial u}{\partial p_{i}} \dot{p}_{i}\right) \\
& =\sum_{i=1}^{n}\left(\frac{\partial u}{\partial q_{i}} \frac{\partial H}{\partial p_{i}}-\frac{\partial u}{\partial p_{i}} \frac{\partial H}{\partial q_{i}}\right)
\end{aligned}
$$

o sea

$$
\frac{\mathrm{d} u}{\mathrm{~d} t}=[u, H]
$$

donde el corchete es el corchete de Poisson entre $u$ y el hamiltoniano $H$. La ecuación (A-31) puede considerarse como la ecuación de movimiento generalizada para una función arbitraria $u$ de la configuración del sistema y, en particular, contiene las propias ecuaciones de Hamilton cuando tomamos por $u$ una de las variables canónicas. Si esta ecuación puede ser integrada para obtener $u(t)$ obtendremos la acción de la transformación canónica buscada. Una solución formal se puede obtener desarrollando $u(t)$ en serie de Taylor en torno al valor inicial $u\left(t_{0}=0\right)$ :

$$
u(t)=u(0)+\left.t \frac{\mathrm{d} u}{\mathrm{~d} t}\right|_{0}+\left.\frac{t^{2}}{2 !} \frac{\mathrm{d}^{2} u}{\mathrm{~d} t^{2}}\right|_{0}+\left.\frac{t^{3}}{3 !} \frac{\mathrm{d}^{3} u}{\mathrm{~d} t^{3}}\right|_{0}+\cdots .
$$

Por la ecuación (A-31) tenemos

$$
\left.\frac{\mathrm{d} u}{\mathrm{~d} t}\right|_{0}=[u, H]_{0},
$$

donde el subíndice cero significa que hay que tomar el valor del corchete de Poisson en el instante inicial. La aplicación reiterada de la ecuación (A-31), tomando al propio $[u, H]$ como función de la configuración del sistema, da

$$
\frac{\mathrm{d}^{2} u}{\mathrm{~d} t^{2}}=[[u, H], H]
$$

y se puede iterar el proceso para obtener la tercera derivada de $u$ y así sucesivamente. La serie de Taylor para $u(t)$ conduce, pues, a la solución formal por serie

$$
u(t)=u(0)+t[u, H]_{0}+\frac{t^{2}}{2 !}[[u, H], H]_{0}+\frac{t^{3}}{3 !}[[[u, H], H], H]_{0}+\cdots .
$$

Una manera fructífera de escribir este desarrollo resulta de considerar el juego de paréntesis de Poisson del término $n$-ésimo como la $n$-ésima aplicación repetida (por la derecha) del operador diferencial lineal

$$
\hat{\mathbf{H}} \equiv[\quad, H] \equiv \sum_{i=1}^{n}\left(\frac{\partial}{\partial q_{i}} \frac{\partial H}{\partial p_{i}}-\frac{\partial}{\partial p_{i}} \frac{\partial H}{\partial q_{i}}\right) .
$$


De esta forma, la ecuación (A-32) adopta la forma

$$
u(t)=e^{t \hat{\mathrm{H}}} u(0)
$$

donde la exponencial define, a partir de su desarrollo en serie, el operador evolución temporal $e^{t \hat{H}}$. Esta ecuación nos dice, pues, que la dependencia temporal de $u$ viene dada por la aplicación del operador evolución temporal sobre su valor inicial.

En principio, todo lo que hemos hecho es reescribir las ecuaciones de movimiento, y podemos percibir que el procedimiento es meramente artificial por cuanto las ecuaciones permanecen sin solución analítica, excepto en casos especiales. Sin embargo esta formulación en términos de los corchetes de Poisson no sólo proporciona una visión profunda de la estructura de la mecánica clásica, sino que, como hemos visto, permite construir integradores numéricos simplécticos. 



\section{Apéndice B}

\section{Variables democráticas heliocéntricas en el problema de $N$-cuerpos}

El hamiltoniano de un sistema de $n=N+1$ partículas de masas $m_{i}(i=0, \ldots, N)$ sujetos únicamente a la acción de sus mutuas atracciones gravitatorias está dado por

$$
H(\mathbf{q}, \mathbf{p})=\sum_{i=0}^{N} \frac{\left|\mathbf{p}_{i}\right|^{2}}{2 m_{i}}-G \sum_{i=0}^{N-1} \sum_{j=i+1}^{N} \frac{m_{i} m_{j}}{\left|\mathbf{q}_{i}-\mathbf{q}_{j}\right|}
$$

donde $\mathbf{q}_{i}=\left(x_{i}, y_{i}, z_{i}\right)$ y $\mathbf{p}_{i}=\left(p_{x_{i}}, p_{y_{i}}, p_{z_{i}}\right)$ son los vectores posición y momento del cuerpo $i$-ésimo (de masa $m_{i}$ ) respecto a un sistema de referencia inercial de coordenadas cartesianas y $G$ es la constante de gravitación universal. Queremos definir un nuevo conjunto de variables canónicas, $\mathbf{Q}_{i}$ y $\mathbf{P}_{i}$, de manera tal que las nuevas posiciones, para $i \neq 0$, sean las coordenadas respecto al cuerpo $i=0$ y la posición del baricentro en el caso $i=0$ :

$$
\mathbf{Q}_{i}= \begin{cases}\mathbf{q}_{i}-\mathbf{q}_{0} & \text { si } i \neq 0 \\ \frac{1}{M} \sum_{j=0}^{N} m_{j} \mathbf{q}_{j} & \text { si } i=0,\end{cases}
$$

donde $M=\sum_{j=0}^{N} m_{j}$ es la masa total del sistema. La transformación canónica que relaciona las variables canónicas nuevas con las originales puede ser construida utilizando la siguiente función generatriz de las nuevas posiciones y los momentos originales (Szebehely, 1967):

$$
F_{3}\left(\mathbf{Q}_{i}, \mathbf{p}_{i}\right)=-\mathbf{p}_{0} \cdot\left(\mathbf{Q}_{0}-\frac{1}{M} \sum_{j=1}^{N} m_{j} \mathbf{Q}_{j}\right)-\sum_{i=1}^{N} \mathbf{p}_{i} \cdot\left(\mathbf{Q}_{i}+\mathbf{Q}_{\mathbf{0}}-\frac{1}{M} \sum_{j=1}^{N} m_{j} \mathbf{Q}_{j}\right) .
$$

Entonces las nuevas variables son canónicas si ${ }^{1}$

$$
\mathbf{q}_{i}=-\frac{\partial F_{3}}{\partial \mathbf{p}_{i}}= \begin{cases}\mathbf{Q}_{i}+\mathbf{Q}_{\mathbf{0}}-\frac{1}{M} \sum_{j=1}^{N} m_{j} \mathbf{Q}_{j} & \text { si } i \neq 0 \\ \mathbf{Q}_{0}-\frac{1}{M} \sum_{j=1}^{N} m_{j} \mathbf{Q}_{j} & \text { si } i=0\end{cases}
$$

\footnotetext{
${ }^{1}$ Véanse la ecuaciones $(\mathrm{A}-14)$ del Apéndice A.
} 
y

$$
\mathbf{P}_{i}=-\frac{\partial F_{3}}{\partial \mathbf{Q}_{i}}= \begin{cases}\mathbf{p}_{i}-\frac{m_{i}}{M} \sum_{j=0}^{N} \mathbf{p}_{j} & \text { si } i \neq 0 \\ \sum_{j=0}^{N} \mathbf{p}_{j} & \text { si } i=0\end{cases}
$$

Así, los nuevos impulsos canónicos son los momentos baricéntricos (si $i \neq 0$ ) y el momento total del sistema (si $i=0$ ). De este modo, la transformación canónica buscada es

$$
\begin{aligned}
& \mathbf{Q}_{i}= \begin{cases}\mathbf{q}_{i}-\mathbf{q}_{0} & \text { si } i \neq 0 \\
\frac{1}{M} \sum_{j=0}^{N} m_{j} \mathbf{q}_{j} & \text { si } i=0,\end{cases} \\
& \mathbf{P}_{i}= \begin{cases}\mathbf{p}_{i}-\frac{m_{i}}{M} \sum_{j=0}^{N} \mathbf{p}_{j} & \text { si } i \neq 0 \\
\sum_{j=0}^{N} \mathbf{p}_{j} & \text { si } i=0 .\end{cases}
\end{aligned}
$$

y su transformación inversa

$$
\begin{aligned}
& \mathbf{q}_{i}= \begin{cases}\mathbf{Q}_{i}+\mathbf{Q}_{\mathbf{0}}-\frac{1}{M} \sum_{j=1}^{N} m_{j} \mathbf{Q}_{j} & \text { si } i \neq 0 \\
\mathbf{Q}_{0}-\frac{1}{M} \sum_{j=1}^{N} m_{j} \mathbf{Q}_{j} & \text { si } i=0,\end{cases} \\
& \mathbf{p}_{i}= \begin{cases}\mathbf{P}_{i}+\frac{m_{i}}{M} \mathbf{P}_{0} & \text { si } i \neq 0 \\
\frac{m_{0}}{M} \mathbf{P}_{0}-\sum_{j=1}^{N} \mathbf{P}_{j} & \text { si } i=0 .\end{cases}
\end{aligned}
$$

Puesto que la transformación canónica de las variables originales a las nuevas no contiene explícitamente al tiempo, el hamiltoniano del sistema respecto de las nuevas variables se obtiene simplemente sustituyendo la transformación (B-7) en la expresión (B-1) de $H$ :

$$
H(\mathbf{Q}, \mathbf{P})=H(\mathbf{q}(\mathbf{Q}, \mathbf{P}), \mathbf{p}(\mathbf{Q}, \mathbf{P}))
$$

Tal sustitución conduce a

$$
H(\mathbf{Q}, \mathbf{P})=\frac{\left|\mathbf{P}_{0}\right|^{2}}{2 M}+\frac{1}{2 m_{0}}\left|\sum_{i=1}^{N} \mathbf{P}_{i}\right|^{2}+\sum_{i=1}^{N}\left(\frac{\left|\mathbf{P}_{i}\right|^{2}}{2 m_{i}}-\frac{G m_{i} m_{0}}{\left|\mathbf{Q}_{i}\right|}\right)-G \sum_{i=1}^{N-1} \sum_{j=i+1}^{N} \frac{m_{i} m_{j}}{\left|\mathbf{Q}_{i}-\mathbf{Q}_{j}\right|}
$$

Puesto que la coordenada $\mathbf{Q}_{0}$ es cíclica, el vector $\mathbf{P}_{0}$ es una constante de movimiento. Esto era de esperarse: el centro de masa del sistema se mueve como una partícula libre y su contribución al hamiltoniano del sistema puede ser ignorada. Así, en términos de las variables democráticas heliocéntricas, el hamiltoniano del sistema toma la forma

$$
H(\mathbf{Q}, \mathbf{P})=H_{\text {Kep }}+H_{\text {int }}+H_{\odot},
$$

donde

$$
\begin{aligned}
H_{\mathrm{Kep}} & =\sum_{i=1}^{N}\left(\frac{\left|\mathbf{P}_{i}\right|^{2}}{2 m_{i}}-\frac{G m_{i} m_{0}}{\left|\mathbf{Q}_{i}\right|}\right), \\
H_{\mathrm{int}} & =-G \sum_{i=1}^{N-1} \sum_{j=i+1}^{N} \frac{m_{i} m_{j}}{\left|\mathbf{Q}_{i}-\mathbf{Q}_{j}\right|}, \\
H_{\odot} & =\frac{1}{2 m_{0}}\left|\sum_{i=1}^{N} \mathbf{P}_{i}\right|^{2} .
\end{aligned}
$$




\section{Apéndice $\mathrm{C}$}

\section{La nebulosa solar de masa mínima}

La nebulosa solar de masa mínima es un modelo de disco protoplanetario que provee una cota inferior a la cantidad de material gaseoso para formar todos los planetas del sistema solar, considerándolos de composición solar. La derivación del mismo procede como sigue (Weidenschilling, 1977; Hayashi, 1981):

I. A partir de las abundancias de elementos pesados (específicamente Fe) de cada planeta (conocidas o modeladas), la masa de cada uno de ellos es aumentada con la adecuada proporción de hidrógeno y helio para lograr una composición semejante a la del Sol (el cual tiene una masa fraccional de hidrógeno $X=0.73$, de helio $Y=0.25$ y "metales" $Z=0.02)$.

II. El sistema solar es dividido en regiones anulares con un planeta por cada anillo. La masa aumentada de cada planeta es distribuida uniformemente en el anillo correspondiente, derivando un perfil de densidad de masa superficial, $\Sigma$, expresado como una ley de potencias de la distancia radial $r$ sobre el plano del disco.

El resultado obtenido es que entre el rango de la órbita de Mercurio a la de Neptuno (pero ignorando el cinturón de asteroides) tal perfil decae como $r^{-3 / 2}$. Típicamente, el perfil supuesto es

$$
\Sigma(r)=10^{3}\left(\frac{r}{1 \mathrm{UA}}\right)^{-3 / 2} \mathrm{~g} \mathrm{~cm}^{-2}
$$

La integración de este perfil entre 0.3 y 30 UA conduce a un valor de la masa mínima de la nebulosa solar de $0.01 M_{\odot}$ (una centésima de la masa solar).

Es importante remarcar que la masa así obtenida es una masa mínima, no es una estimación de la masa presente al tiempo de la formación del disco protoplanetario solar. Además, el perfil obtenido, $\Sigma \propto r^{-3 / 2}$, no necesariamente constituye un perfil característico para todos los discos protoplanetarios. Otros modelos teóricos predicen un perfil $\Sigma \propto r^{-1}$, el cual decae más lentamente con la distancia. 



\section{Apéndice D}

\section{Condición para las resonancias de Lindblad y de corrotación}

La siguiente exposición está basada en el correspondiente tópico del libro Galactic dynamics de Binney y Tremaine (1987). Consideremos un planeta orbitando una estrella sobre una órbita circular de frecuencia angular constante $\Omega_{p}$. Tomando un sistema de coordenadas $(x, y, z)$ con origen en el centro de masas del sistema, con el plano $x-y$ como el plano de dicha órbita, y que rota con velocidad angular $\Omega_{p}=\Omega_{p} \hat{\mathbf{k}}$, siendo $\Omega_{p}>0$, la ecuación de movimiento para el vector posición $\mathbf{r}$ de una partícula de prueba de masa despreciable respecto de este sistema rotante de coordenadas es:

$$
\ddot{\mathbf{r}}=-\nabla \phi-2\left(\boldsymbol{\Omega}_{p} \times \dot{\mathbf{r}}\right)-\boldsymbol{\Omega}_{p} \times\left(\boldsymbol{\Omega}_{p} \times \mathbf{r}\right)
$$

donde $\phi$ es el potencial gravitatorio y los términos $-2\left(\boldsymbol{\Omega}_{p} \times \dot{\mathbf{r}}\right)$ y $-\boldsymbol{\Omega}_{p} \times\left(\boldsymbol{\Omega}_{p} \times \mathbf{r}\right)$ corresponden a las fuerzas de Coriolis y centrífuga, respectivamente. Reduciendo el problema al plano en que se mueve el planeta, podemos considerar coordenadas polares $(r, \theta)$ sobre el mismo, y la ecuación de movimiento anterior toma la forma

$$
\begin{aligned}
\ddot{r}-r \dot{\theta}^{2} & =-\frac{\partial \phi}{\partial r}+2 r \dot{\theta} \Omega_{p}+\Omega_{p}^{2} r, \\
r \ddot{\theta}+2 \dot{r} \dot{\theta} & =-\frac{1}{r} \frac{\partial \phi}{\partial \theta}-2 \dot{r} \Omega_{p} .
\end{aligned}
$$

Suponiendo que la masa del planeta es mucho menor que la estelar, podemos escribir el potencial $\phi$ como el potencial central estelar más una contribución perturbativa debida al planeta:

$$
\phi(r, \theta)=\phi_{0}(r)+\phi_{1}(r, \theta)
$$

donde $\left|\phi_{1} / \phi_{0}\right| \ll 1$ en tanto la partícula de prueba no esté dentro de la esfera de influencia del planeta. En esta situación podemos representar el movimiento de la partícula de prueba como desviaciones debidas a la perturbación $\phi_{1}$ del movimiento en el potencial central $\phi_{0}$ :

$$
r(t)=r_{0}+r_{1}(t), \quad \theta(t)=\theta_{0}(t)+\theta_{1}(t)
$$




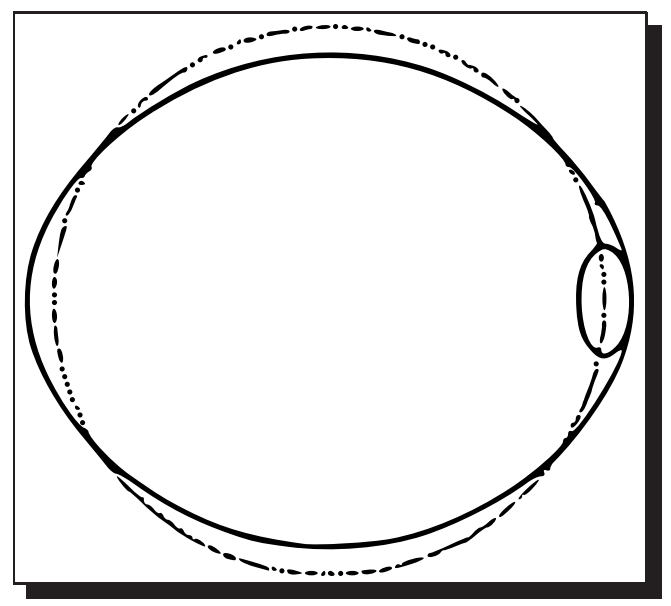

Figura D.1. En la aproximación epicíclica el movimiento de una partícula de prueba sobre una órbita elíptica (curva continua) es obtenida superponiendo al movimiento del centro de guía, en órbita circular con velocidad angular $\Omega_{0}-\Omega_{p}$ (órbita punteada), un epiciclo elíptico alrededor del mismo.

En la literatura esta descripción del movimiento es conocida como aproximación epicíclica, por cuanto el movimiento orbital de la partícula de prueba es obtenido superponiendo al movimiento del punto $\left(r_{0}, \theta_{0}\right)$, denominado el centro de guía, las desviaciones $\left(r_{1}, \theta_{1}\right)$ respecto del mismo (ver Fig. D.1).

Puesto que aun para una perturbación nula deben satisfacerse las ecuaciones de movimiento, se sigue de la ec. (D-2a) que

$$
-r_{0} \dot{\theta}_{0}^{2}=-\left.\frac{\mathrm{d} \phi_{0}}{\mathrm{~d} r}\right|_{r_{0}}+2 r_{0} \dot{\theta}_{0} \Omega_{p}+\Omega_{p}^{2} r_{0},
$$

la cual conduce a la usual ecuación para el equilibrio centrífugo en $r_{0}$ :

$$
r_{0}\left(\dot{\theta}_{0}+\Omega_{p}\right)^{2}=\left.\frac{\mathrm{d} \phi_{0}}{\mathrm{~d} r}\right|_{r_{0}} .
$$

Introduciendo la frecuencia circular a la distancia $r$ en el potencial $\phi_{0}$ :

$$
\Omega=\sqrt{\frac{1}{r} \frac{\mathrm{d} \phi_{0}}{\mathrm{~d} r}}
$$

la ecuación anterior permite escribir la velocidad angular del centro de guía como

$$
\dot{\theta}_{0}=\Omega_{0}-\Omega_{p}
$$

donde $\Omega_{0}=\Omega\left(r_{0}\right)$. Escogido el origen del tiempo de manera tal que $\theta_{0}(t=0)=0$, resulta

$$
\theta_{0}=\left(\Omega_{0}-\Omega_{p}\right) t
$$

Al reemplazar las ecs. (D-3) y (D-4) en las ecuaciones de movimiento (D-2) y retener 
sólo los primeros ordenes en $r_{1}$ y $\dot{\theta}_{1}$, obtenemos las ecuaciones

$$
\begin{aligned}
\ddot{r}_{1}+\left.\left(\frac{\mathrm{d}^{2} \phi_{0}}{\mathrm{~d} r^{2}}-\Omega^{2}\right)\right|_{r_{0}} r_{1}-2 r_{0} \Omega_{0} \dot{\theta}_{1} & =-\left.\frac{\partial \phi_{1}}{\partial r}\right|_{r_{0}}, \\
\ddot{\theta}_{1}+2 \Omega_{0} \frac{\dot{r}_{1}}{r_{0}} & =-\left.\frac{1}{r_{0}^{2}} \frac{\partial \phi_{1}}{\partial \theta}\right|_{r_{0}} .
\end{aligned}
$$

Para continuar debemos explicitar la forma del potencial perturbador $\phi_{1}$. Es ampliamente conocido que, en virtud de los procedimientos clásicos de la Mecánica Celeste, el mismo puede ser desarrollado en una serie de términos cosenoidales cuyos argumentos son múltiplos de $\theta$,

$$
\phi_{1}(r, \theta)=\sum_{m} A_{m}(r) \cos (m \theta)
$$

donde $m$ es un entero (ver, por ejemplo, Danby, 1992). Debido a que los términos cosenoidales contribuyen a través de una serie, podemos considerar, sin pérdida de generalidad, un único miembro arbitario del mismo. Así, explicitamos el potencial perturbativo como

$$
\phi_{1}(r, \theta)=A_{m}(r) \cos (m \theta),
$$

para un dado entero $m$. Suponiendo que $\theta_{1}$ se mantiene pequeño, podemos aproximar $\theta$ por $\theta_{0}=\left(\Omega_{0}-\Omega_{p}\right) t$ en la ecuación anterior, y nos permite derivar a partir de (D-10), las ecuaciones

$$
\begin{aligned}
\ddot{r}_{1}+\left.\left(\frac{\mathrm{d}^{2} \phi_{0}}{\mathrm{~d} r^{2}}-\Omega^{2}\right)\right|_{r_{0}} r_{1}-2 r_{0} \Omega_{0} \dot{\theta}_{1} & =-\left.\frac{\mathrm{d} A_{m}}{\mathrm{~d} r}\right|_{r_{0}} \cos \left[m\left(\Omega_{0}-\Omega_{p}\right) t\right], \\
\ddot{\theta}_{1}+2 \Omega_{0} \frac{\dot{r}_{1}}{r_{0}} & =\frac{m A_{m}\left(r_{0}\right)}{r_{0}^{2}} \operatorname{sen}\left[m\left(\Omega_{0}-\Omega_{p}\right) t\right] .
\end{aligned}
$$

La integración de la segunda de estas ecuaciones conduce a

$$
\dot{\theta}_{1}=-2 \Omega_{0} \frac{r_{1}}{r_{0}}-\frac{A_{m}\left(r_{0}\right)}{r_{0}^{2}\left(\Omega_{0}-\Omega_{p}\right)} \cos \left[m\left(\Omega_{0}-\Omega_{p}\right) t\right]+\text { constante }
$$

ecuación que permite eliminar $\dot{\theta}_{1}$ en (D-13a) para obtener, finalmente

$$
\ddot{r}_{1}+\kappa_{0}^{2} r_{1}=-\left[\frac{\mathrm{d} A_{m}}{\mathrm{~d} r}+\frac{2 \Omega A_{m}}{r\left(\Omega-\Omega_{p}\right)}\right]_{r_{0}} \cos \left[m\left(\Omega_{0}-\Omega_{p}\right) t\right]+\text { constante }
$$

donde $\kappa_{0}$, la denominada frecuencia epicíclica, es definida por

$$
\kappa_{0}^{2} \equiv\left[\frac{\mathrm{d}^{2} \phi_{0}}{\mathrm{~d} r^{2}}+3 \Omega^{2}\right]_{r_{0}} .
$$

Nótese que para un potencial kepleriano, $\phi_{0}=-\mu / r$, resulta $\kappa_{0}=\Omega_{0}=\sqrt{\mu / r_{0}^{3}}$.

En la ecuación (D-15) la constante involucrada carece de importancia puesto que puede ser absorbida por el desplazamiento $r_{1} \rightarrow r_{1}+$ constante. De este modo, la 


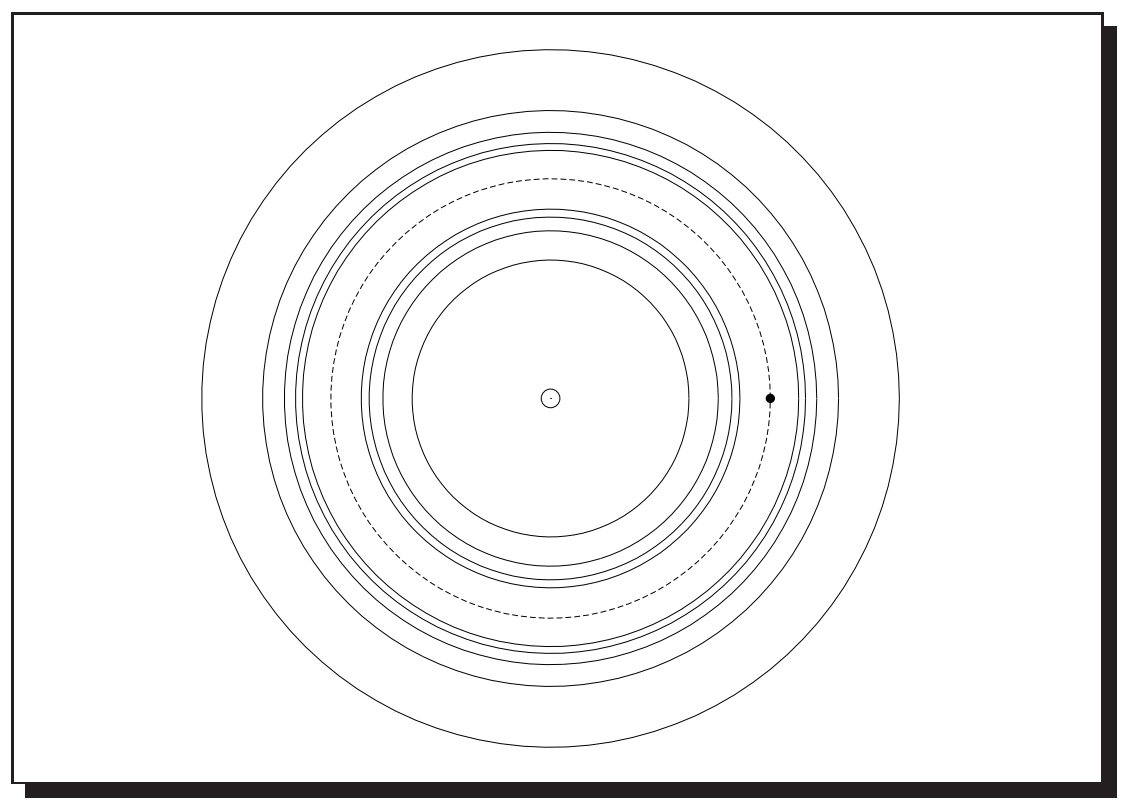

Figura D.2. Localización de la resonancia de corrotación (línea punteada) y de las resonancias de Lindblad (líneas continuas) para un planeta en órbita circular en el potencial kepleriano de una estrella central. El gráfico muestra las resonancias de más bajo orden, a saber, según su proximidad al planeta, $m=5,4,3,2,1$.

misma es la ecuación de un oscilador armónico de frecuencia natural $\kappa_{0}$ forzado por una perturbación de frecuencia $m\left(\Omega_{0}-\Omega_{p}\right)$. La solución general de esta ecuación es

$$
r_{1}(t)=C_{1} \cos \left(\kappa_{0} t+\delta\right)-\left[\frac{\mathrm{d} A_{m}}{\mathrm{~d} r}+\frac{2 \Omega A_{m}}{r\left(\Omega-\Omega_{p}\right)}\right]_{r_{0}} \frac{\cos \left[m\left(\Omega_{0}-\Omega_{p}\right)\right]}{\kappa_{0}^{2}-m^{2}\left(\Omega_{0}-\Omega_{p}\right)^{2}}
$$

siendo $C_{1}$ y $\delta$ constantes fijadas por las condiciones iniciales. De esta ecuación es claro que existen ciertos valores de $r_{0}$ de la órbita no perturbada que conducen a valores singulares de la órbita epicíclica. A saber:

I. En la denominada resonancia de corrotación, cuando

$$
\Omega_{0}=\Omega_{p}
$$

En esta situación, según la ecuación (D-8), la velocidad angular del centro de guía es nula. Es decir, el centro de guía corrota con el potencial perturbador.

II. En las denominadas resonancias de Lindblad, cuando

$$
m\left(\Omega_{0}-\Omega_{p}\right)= \pm \kappa_{0}
$$

El signo positivo corresponde a una resonancia interna de Lindblad donde $\Omega_{0}>\Omega_{p}$, es decir el centro de guía, y por ende la partícula de prueba (puesto que hemos supuesto que su órbita es muy próxima a la no perturbada) rota más rápido que el potencial perturbador. El signo negativo, por su parte, corresponde a una resonancia externa de Lindlad, donde el centro de guía rota más lento que el potencial perturbador. Para un potencial kepleriano, $\phi=-\mu / r$, donde además la masa del cuerpo central domina sobre 
la del planeta y cualquier otro cuerpo, la ecuación anterior define las localizaciones de las resonancias:

$$
r_{0}=\left(1 \pm \frac{1}{m}\right)^{2 / 3} r_{p}
$$

donde $r_{p}$ es el radio orbital del planeta. La Figura D.2 ilustra la localización de las mismas para las resonancias de orden más bajo. 



\section{Apéndice E}

\section{Zona de cruce planetaria}

Consideremos un planeta en una órbita circular de radio $a_{p}$ y otro cuerpo con una órbita tal que la distancia al perihelio $q=a(1-e)$ y la distancia al afelio $Q=a(1+e)$, siendo $a$ el semieje mayor y $e$ la excentricidad de dicha órbita, cumplan las condiciones

$$
q=a(1-e)<a_{p}<Q=a(1+e)
$$

Bajo estas condiciones las dos órbitas pueden tener un punto en común (para verificar si lo tienen debería considerarse la geometría en el espacio, puesto que las órbitas pueden estar inclinadas y con diferentes argumentos del perihelio). Las desigualdades anteriores, junto con las restricciones $0 \leq e \leq 1$ propias de la definición de la excentricidad,

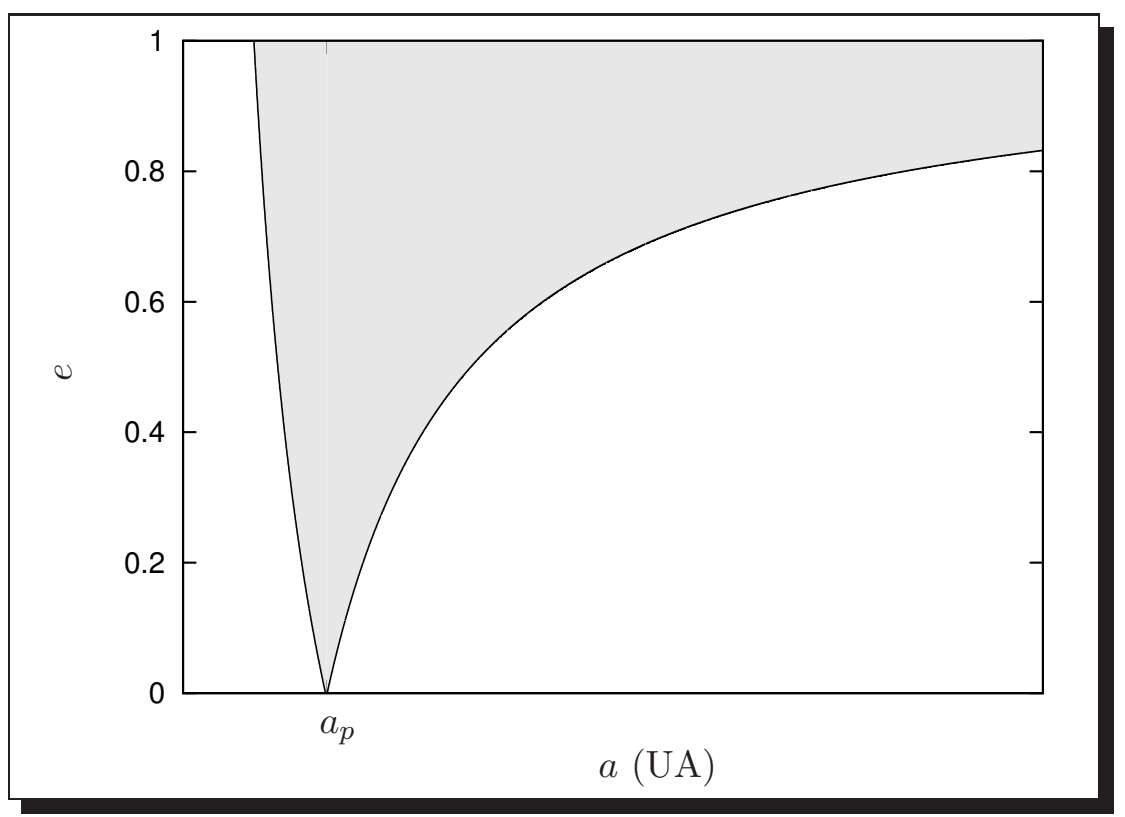

Figura E.1. Zona de cruce de un planeta con órbita circular de radio $a_{p}=5$ en el plano $a-e$. 
definen entonces una región en el plano a-e conocida como zona de cruce del planeta:

$$
e> \begin{cases}\frac{a_{p}}{a}-1, & \text { si } a_{p} / 2 \leq a \leq a_{p} \\ 1-\frac{a_{p}}{a}, & \text { si } a_{p}<a<\infty\end{cases}
$$

La Fig. E.1 ilustra dicha región. 


\section{Apéndice F}

\section{Evolución de la velocidad aleatoria en el modelo "partícula en una caja"}

La siguiente discusión está basada en el artículo de Lissauer y Stewart (1993). Las trayectorias de los planetesimales del disco son órbitas aproximadamentes keplerianas alrededor del cuerpo central, y, como tales, pueden ser descritas por los elementos orbitales de sus órbitas osculatrices (esto es, la órbita kepleriana que tendría el cuerpo, en cada instante de tiempo, si todas las perturbaciones desaparecieran súbitamente). El modelo estadístico de "partícula en una caja" describe el movimiento de los planetesimales con una velocidad relativa aleatoria. La relación entre esta velocidad aleatoria y los elementos orbitales no es directa y distintas expresiones pueden ser utilizadas dependiendo del aspecto específico a considerar. De hecho, a lo largo de esta tesis, utilizamos dos relaciones, las cuales, ambas, suponen que tanto la excentricidad $e$ como la inclinación $i$ de las órbitas son pequeñas:

I. La velocidad del planetesimal relativa a la órbita circular en el plano del disco con el mismo semieje mayor que el del planetesimal:

$$
v=\left(e^{2}+i^{2}\right)^{1 / 2} v_{\mathrm{K}},
$$

siendo $v_{\mathrm{K}}$ la velocidad kepleriana de la órbita de referencia.

II. La velocidad del planetesimal relativa a la órbita circular local:

$$
v=\left(\frac{5}{8} e^{2}+\frac{1}{2} i^{2}\right)^{1 / 2} v_{\mathrm{K}},
$$

siendo $v_{\mathrm{K}}$ la velocidad kepleriana de la órbita de referencia.

La segunda definición corresponde a la velocidad aleatoria descrita en el modelo de "partícula en una caja". La tasa de cambio de la velocidad aleatoria $V$ de un cuerpo de masa $M$ embebido en el disco de planetesimales de masas $m$ está dada por

$$
\frac{\mathrm{d} V}{\mathrm{~d} t}=A+B+C+D
$$

donde los términos de esta ecuación están dados como sigue. 
A. Excitación de la velocidad aleatoria causada por encuentros gravitacionales:

$$
A=\frac{3}{4} \frac{\sqrt{\pi} G^{2}}{V \mathcal{V}^{3}} \rho \log \Lambda\left[(9 L-12 \sqrt{3})(M+m) V^{2}+(5 L-4 \sqrt{3})\left(m v^{2}-M V^{2}\right)\right] .
$$

siendo $\rho$ la densidad volumétrica de masa del planetesimal de masa $m$,

$$
\begin{aligned}
\mathcal{V}^{2} & \equiv V^{2}+v^{2} \\
L & \equiv \log [(2+\sqrt{3}) /(2-\sqrt{3}) \approx 2.634 \\
\Lambda & \equiv \frac{\operatorname{sen}\left(\Psi_{\operatorname{máx}} / 2\right)}{\operatorname{sen}\left(\Psi_{\min } / 2\right)}
\end{aligned}
$$

donde el ángulo de deflexión $\Psi$ está relacionado con el parámetro de impacto $b$ según la fórmula de dispersión de Rutherford:

$$
\operatorname{sen}\left(\frac{\Psi}{2}\right)=\left(1+\frac{b^{2}}{b_{0}^{2}}\right)^{-1 / 2}
$$

siendo $b_{0}=G(M+m) \mathcal{V}^{-2}$. El ángulo de deflexión mínimo $\Psi_{\text {mín }}$ es calculado con el parámetro de impacto

$$
b=b_{\text {máx }}=\operatorname{máx}(V / \Omega, v / \Omega)
$$

siendo $\Omega$ la frecuencia orbital, en tanto que el ángulo de deflexión máximo $\Psi_{\text {máx }}$, es calculado utilizando el parámetro de impacto correspondiente a la sección eficaz de captura gravitacional $R_{g}$ en la formulación del problema de dos cuerpos,

$$
b=b_{\text {mín }}=R_{g}=\left(R_{\mathrm{M}}+R_{\mathrm{m}}\right)\left[1+\frac{2 b_{0}}{R_{\mathrm{M}}+R_{\mathrm{m}}}\right]^{1 / 2},
$$

donde $R_{\mathrm{M}}$ y $R_{\mathrm{m}}$ son los radios físicos de los cuerpos de masa $M$ y $m$ respectivamente. De este modo,

$$
\Lambda=\left[\frac{b_{\text {máx }}^{2}+b_{0}^{2}}{R_{g}^{2}+b_{0}^{2}}\right]^{1 / 2}
$$

$B$. Excitación de la velocidad aleatoria originada por colisiones inelásticas,

$$
B=\frac{\sqrt{\pi}}{8}\left(\sqrt{3}-\frac{5 L}{12}\right) \frac{\mathcal{V}}{V} \rho R_{g}^{2} \frac{m\left(V^{2}-v^{2}\right)+2 M V^{2}}{(M+m)^{2}} .
$$

$C$. Amortiguamiento de la velocidad debido a la disipación de energía por colisiones inelásticas,

$$
C=-\sqrt{\pi}\left(\frac{11 \sqrt{3}}{18}+\frac{L}{24}\right) \frac{\mathcal{V}}{V} \rho R_{g}^{2} \frac{m\left(V^{2}-v^{2}\right)+2 M V^{2}}{(M+m)^{2}}
$$

D. Transferencia de energía de los cuerpos mayores a los más pequeños vía fricción dinámica,

$$
D=\frac{4 \sqrt{\pi} L G^{2}}{V \mathcal{V}^{3}} \rho \log \Lambda\left(m v^{2}-M V^{2}\right) .
$$




\section{Bibliografía}

[1] Armitage, P.J., 2010, Astrophysics of Planet Formation, Cambridge University Press, Cambridge, EE.UU.

[2] Barnes, J.E., 1995, en: The Formation of Galaxies, Cambridge University Press, Cambridge, EE.UU., 399.

[3] Barnes, J.E. y Hut, P., 1986, Nat, 324, 446.

[4] Barnes, J.E. y Hut, P., 1989, ApJS, 70, 389.

[5] Binney, J. y Tremaine. S., 1987, Galactic Dynamics, Princeton University Press, Princeton, EE. UU.

[6] Brunini, A. y Melita, M.D., 2002, Icarus, 160, 32.

[7] Brunini, A. y Viturro, H., 2003, Mon. Not. R. Astron. Soc., 346, 924.

[8] Chambers, J.E., 1999, Mon. Not. R. Astron. Soc., 304, 793.

[9] Chamber, J.E., Migliorini F., 1997, BAAS, 29, 1024.

[10] Chandrasekhar S., 1942, Principles of stellar dynamics. The Univ. of Chicago Press, Chicago, EE.UU..

[11] Cionco, R.G. y Brunini, A., 2002, Mon. Not. R. Astron. Soc., 334, 77.

[12] Cole, H.A. y Woolfson, M.M., 2002, Planetary Science, Institute of Physics Publishing, Bristol, UK.

[13] Danby, J.M.A., 1992, Fundamentals of Celestial Mechanics, Second Edition, Willmann-Bell, Inc., Richmond, EE.UU.

[14] Davies, J.J., McFarland, J., Bailey M.E., Mardsden, B. e Ip, W.H, 2008, en: The Kuiper Belt, University of Arizona Space Science Series.

[15] Delsanti, A. yJewitt, D., 2006, en Solar System Update, Eds: P. Blondel y J. Mason, Springer, 267.

[16] Duncan, M.J., Levison, H.F. y Lee, M.H., 1988, AJ, 116, 2067.

[17] Fernández, J.A. e Ip, W.H., 1984, Icarus, 58, 109. 
[18] Goldreich P., Tremaine S., 1979, ApJ, 233, 857.

[19] Goldreich P. y Tremaine S., 1980, ApJ, 241, 425.

[20] Goldstein, H., 1990, Mecánica Clásica, Segunda edición, Editorial Reverté, Barcelona, España.

[21] Gomes, R., Morbidelli, A. y Levison, H. F. 2004, Icarus, 170, 492.

[22] Gomes, R., Levison, H.F., Tsiganis y K., Morbidelli, A., 2005, Nature, 435, 466.

[23] Hahn, J.M, 2003, Astroph. J., 595, 531.

[24] Hayashi, C., 1981, Prog. Theor. Phys. Suppl., 70, 35.

[25] Hernquist L., 1987, ApJS, 64, 715.

[26] Jewitt, D., y Luu, J., 1993, Nature, 362, 730.

[27] Kinoshita, H., Yoshida y H., Nakai, H., 1991, Celest. Mech. Dyn. Astron., 50, 59.

[28] Kokubo, E. y Ida, S., 1996, Icarus 123, 180.

[29] Kortenkamp, S. J., Kokubo, E., y Weidenschilling, S.J., 2000, Formation of planetary embryos. Origin of the Earth and Moon, 1, 85-100.

[30] Ida S. y Makino J., 1993, Icarus, 106, 210.

[31] Levison, H.F. y Duncan, M.J., 1994, Icarus 108, 1836.

[32] Levison, H.F., Morbidelli, A., VanLaerhoven, C., Gomes, R. y Tsiganis, K., 2008, Icarus, 196, 258.

[33] Lin, D.N.C., e Ida, S., 1997, ApJ, 477, 781.

[34] Lin, D.N.C., Bodenheimer, P. y Richardson, D. C., 1996, Nature, 380, 606.

[35] Lissauer, J.J., Stewart, G.R., 1993, en: Protostars and Planets III, Eds: E. Levy y J. Lunine, University of Arizona, Space Science Series, p. 1061.

[36] Lynden-Bell D. y Kalnajs A.J., 1972, Mon. Not. R. Astron. Soc., 157, 1.

[37] Lucas, P.W., y Roche, P.F., 2000, Mon. Not. R. Astron. Soc., 314,4.

[38] Malhotra, R., 1993, Nature, 365, 819.

[39] Malhotra, R., 1995, Astron. J., 110, 420.

[40] Morbidelli, A., Levison, H.F., Tsiganis, K. y Gomes, R., 2005, Nature, 435, 462.

[41] Morbidelli, A., Tsiganis, K., Crida, A., Levison H.F. y Gomes, R., 2007, AJ, 134, 1790.

[42] Murray-Clay, R.A. y Chiang E.I., 2005, AJ, 619, 623. 
[43] Murray, C. y Dermott, S., 1999, Solar System Dynamics, Cambridge University Press, Cambridge, EE.UU.

[44] Pfalzner, S. y Gibbon, P., 1996, Many-Body Tree Methods in Physics, Cambridge University Press, Cambridge, EE.UU.

[45] Rasio, F.A., y Ford, E.B., 1996, Science, 274, 954.

[46] Safronov, V.S., 1969, Evolution of the protoplanetary cloud and formation of the Earth and the Planets, NASA TTF-677.

[47] Santamaría, P.J., 2004, Un código de árbol para la dinámica de planetesimales, Tesis de licenciatura, FCAG, UNLP.

[48] Stewart y G.R., Wetherill, G.W., 1988, Icarus 74, 542.

[49] Szebehely, V. 1967, Theory of Orbits, New York, Academic.

[50] Tanaka, H.,. Takeuchi, T., y Ward, W.R., 2002, ApJ, 565, 1257.

[51] Tera, F., Papanastassiou, D.A. y Wasserburg, G.J., 1974, Earth and Planetary Science Letters, 22, 1.

[52] Tsiganis, K., Gomes, R., Morbidelli, A., y Levison, H.F., 2005, Nature 435, 459.

[53] Ward, W.R., 1997, Icarus, 126, 261.

[54] Ward, W.R. y Hahn, J.M., 1998, AJ, 116, 489.

[55] Ward, W.R. y Hahn, J.M., 2003, AJ, 125, 3389.

[56] Wetherill, G.W. y Stewart, G.R., 1989, Icarus, 77, 330.

[57] Weidenschilling, S.J. 1977, Mon. Not. R. Astron. Soc., 180, 57.

[58] Weidenschilling, S.J., y Marzari, F. 1996, Nature, 384, 619.

[59] Yoshida, H., 1993, Celest. Mech. Dyn. Astron., 56, 27. 

Sobre la interacción planeta-disco

en discos de planetesimales.

Pablo J. Santamaría.

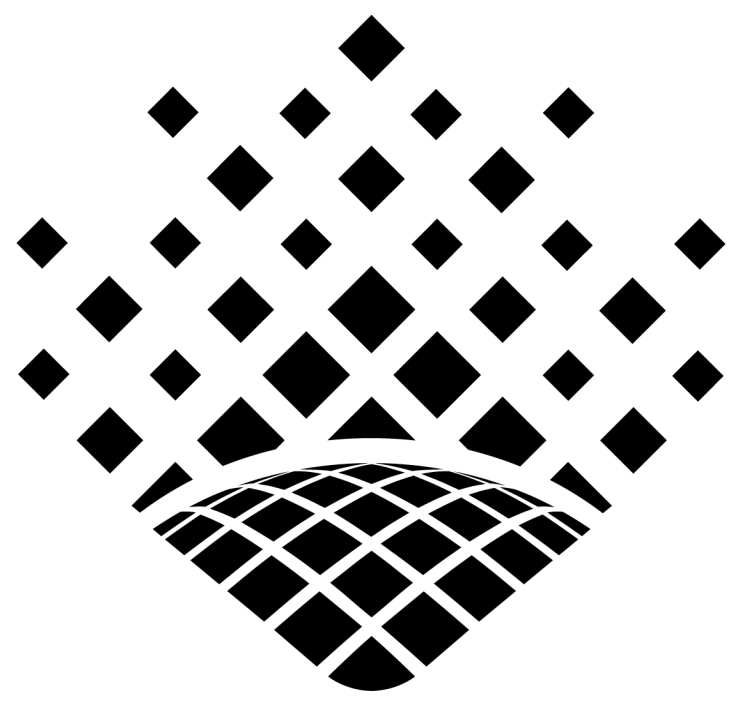

Facultad de Ciencias Astronómicas y Geofísicas

UNIVERSIDAD NACIONAL DE LA PLATA 